\title{
UCRL-TR-225239
}

LA W REN CE LIVERMORE NATIONAL LABORATORY
Gamma-ray Imaging Methods

K. Vetter, L. Mihailescu, K. Nelson, J. Valentine,

D. Wright

October 12, 2006 
This document was prepared as an account of work sponsored by an agency of the United States government. Neither the United States government nor Lawrence Livermore National Security, LLC, nor any of their employees makes any warranty, expressed or implied, or assumes any legal liability or responsibility for the accuracy, completeness, or usefulness of any information, apparatus, product, or process disclosed, or represents that its use would not infringe privately owned rights. Reference herein to any specific commercial product, process, or service by trade name, trademark, manufacturer, or otherwise does not necessarily constitute or imply its endorsement, recommendation, or favoring by the United States government or Lawrence Livermore National Security, LLC. The views and opinions of authors expressed herein do not necessarily state or reflect those of the United States government or Lawrence Livermore National Security, LLC, and shall not be used for advertising or product endorsement purposes.

This work performed under the auspices of the U.S. Department of Energy by Lawrence Livermore National Laboratory under Contract DE-AC52-07NA27344. 


\section{Gamma-ray Imaging Methods}

Kai Vetter, Lucian Mihailescu, Karl Nelson, John Valentine, Douglas Wright

Lawrence Livermore National Laboratory

September 29, 2006

UCRL-X

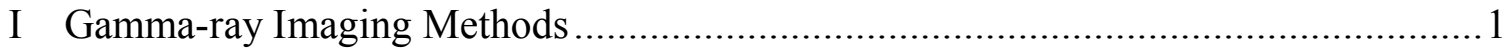

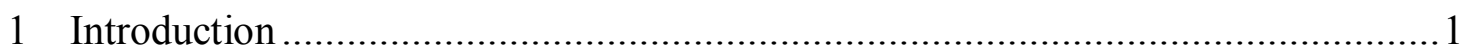

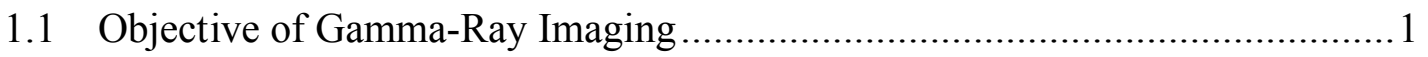

1.2 Relevance to Search and Surveillance Applications........................................ 4

1.3 Distinctions Among Imaging/Directional Detectors ...................................... 8

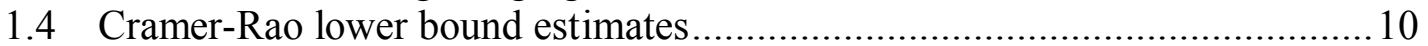

2 Overview of Gamma-Ray Imaging Concepts ……......................................... 12

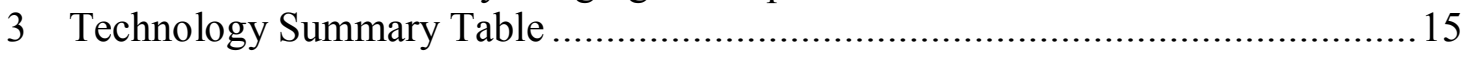

II Appendix: Gamma-ray Imaging Concept Details ………………….................... 17

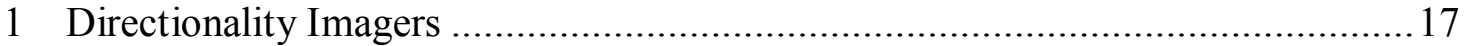

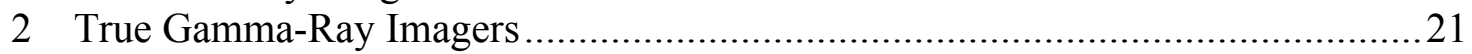

2.1 Conventional, collimator-based systems ......................................................2

2.2 Advanced, modulation systems …………………................................26

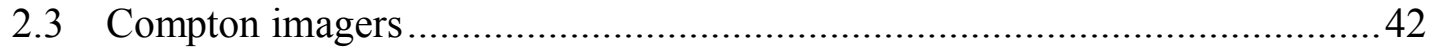

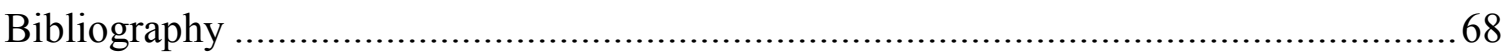




\section{Gamma-RAY IMAGING MethodS}

\section{Introduction}

In this document we discuss specific implementations for gamma-ray imaging instruments including the principle of operation and describe systems which have been built and demonstrated as well as systems currently under development. There are several fundamentally different technologies each with specific operational requirements and performance trade offs.

We provide an overview of the different gamma-ray imaging techniques and briefly discuss challenges and limitations associated with each modality (in the appendix we give detailed descriptions of specific implementations for many of these technologies). In Secition 3 we summarize the performance and operational aspects in tabular form as an aid for comparing technologies and mapping technologies to potential applications.

\subsection{Objective of Gamma-Ray Imaging}

The imaging of gamma-ray photons - similarly e.g. to photons in the visible wavelength domain - provides the ability to determine the origin of photons in space. To date, this ability has been predominantly used in medical applications to trace specific radioactive markers in humans and animals and more recently in astrophysics applications. The goal in medical applications is to obtain information on transport, distribution, metabolic and clearance processes in general, or more specifically, for instance to detect cancer or to study certain dynamical behavior, such as drug addition. From the first Anger camera in 1959 [1], which allowed to obtain planar images, to the current state-of-the art tomographic systems, many advances have been introduced, particularly with respect to the ability to reconstruct images in three dimensions with improved contrast, resolution, and less artifacts. However, although many advances have been implemented, the overall approach is still the same as it was more than 40 years ago, that is to use a collimator to project an image on to a two-dimensionally position-sensitive scintillator such $\mathrm{NaI}(\mathrm{Tl})$. The goal of gamma-ray imaging in astrophysics is primarily to map gamma-ray energy spectra to certain locations in space, e.g. known locations of supernovae events to study the evolution of stars and the synthesis of nuclei.

Important properties of a gamma-ray imager are the ability to localize and to measure the shape of the tracer distributions with high spatial resolution, high efficiency, and high contrast. In nuclear medicine, these three factors characterize image quality and are expressed as [2]: 
1. Spatial resolution, i.e. detail or sharpness of an image object;

2. Contrast, i.e. representation of activity concentration differences in object; and

3. Noise, i.e. statistical noise due to random fluctuations in radioactive decay or systematic noise, e.g. due to the instrumental artifacts.

Although these factors describe three different aspects of image quality, they cannot be treated as completely independent parameters because improvements in one of them are frequently obtained at the expense or degradation of one or more of the others. For example, improved spatial resolution of the collimator usually involves a tradeoff of decreased collimator efficiency and hence decreased counting rates and increased image statistical noise. In nuclear medicine as for instance in X-ray imaging, the image quality cannot only be measured by physical means as discussed before, but an important component is how well an observer or operator can detect and identify a specific object in the image. Although related to physical measures of image quality, the relationships are not well-established due to the complexity of the human visual system and other factors, such as the experience of the observer. In the context of gamma-ray imaging in this study, we neglect the second, operator dependent aspect and focus on the physical measure to characterize and compare gamma-ray imaging instruments.

Two more quantities are of importance for gamma-ray imaging and the detection and identification of nuclear materials, which are not as important for medical applications: the field-of-view (FOV) and the energy resolution: The FOV defines the solid angle, which can be seen and imaged by the instrument. In nuclear medicine, the object of interest is generally close to the detection system, for instance sensitivities and resolution values are normally stated at $10 \mathrm{~cm}$ distance between the object and the collimator. For Anger cameras in the typical parallel-hole collimator configuration, the FOV is roughly defined by the area of the camera projected into the object. The goal is to resolve structures within this area. However, for larger distances, the resolution of such a system degrades to a point at which no structures can be resolved within the FOV since the gamma-ray source is seen through all holes of the collimator. The system then serves only as a tube-like collimator, which provides imaging capabilities only by moving the system.

Figure 1: Illustration of parallel-hole imaging performance at near field (a.)

a.)

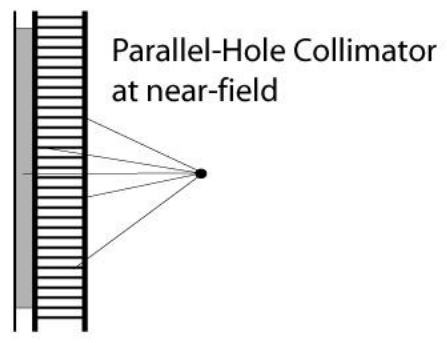

b.)

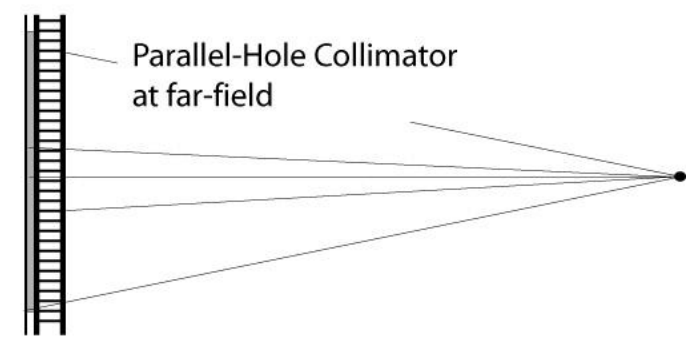

c.) Tube-Hole Collimator at far-field

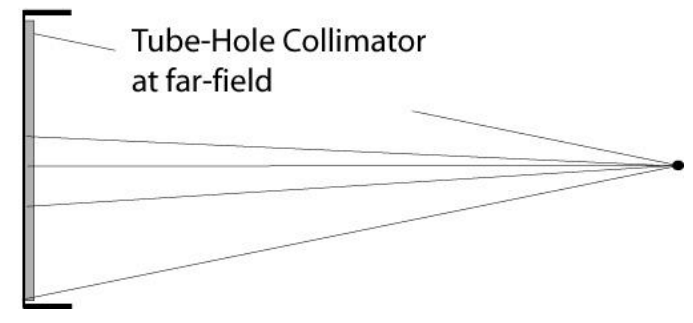


and far field (b. and c.). At near field - common in nuclear medicine applications - the gamma rays from the objects can only be seen through a few holes, the majority of impinging gamma rays are blocked in the collimator front face or the septa. The septa loose their purpose if the source is moved far away with respect to the septa thickness, e.g. the incident gamma rays can be treated as parallel. This implies that no structure can be resolved within the FOV. Therefore, the parallel-hole collimator serves only as a tube collimator shown in c.), however degrades the performance since the collimator still attenuates most of the incident gamma rays.

The two extremes in gamma-ray imaging with regard to the FOV are the Compton imagers and gamma-ray optics systems. Compton imaging enables, in principle, omnidirectional gamma-ray imaging; a gamma-ray optics instrument is characterized by very small FOV since gamma rays can only be imaged through very small diffractive scattering angles.

The energy resolution defines how well individual gamma-ray lines in an energy spectrum can be distinguished from other lines and background. Since in medical applications, the radioisotope and therefore the gamma-ray energies are known, the energy resolution is primarily used to distinguish between gamma rays, which were scattered in the object or in the surrounding materials and gamma rays which enter the camera non-scattered with their originally emitted energy deposited in the detector. Only the directions reconstructed from non-scattered gamma rays point to the true emission location. Not being able to distinguish between these two scattered and unscattered photons can significantly increase the blurring in images. However, in contrast to medical applications, in most other applications, the radioisotopes are not known a priori and the energy resolution is thus very important to optimize the ability to identify the emitting radioisotope.

In addition to application in nuclear medicine, gamma-ray imaging instruments have been developed for astrophysics applications, which are either deployed on short balloon flights or longer space flights. The goal of gamma-ray imaging for astrophysics is to study nuclear processes, for example, in the formation and evolution of stars. As in nuclear medicine, the challenge is to distinguish gamma rays from a specific object of interest (e.g. a particular Supernova), from background radiation, which is caused by all the other nuclear processes in the cosmos. Gamma-ray imaging provides the ability to "zoom" into a particular stellar constellation and enables the measurement of gamma-ray energy spectra from these locations. Ultimately, the position, intensity, and shape of lines in an energy spectrum provide the information needed to better understand the nuclear processes in stars and the environment in which these processes occur.

A third - yet less established - area for gamma-ray imaging was identified and is being pursued for nuclear safeguards applications with the goal to monitor nuclear facilities and materials and to diagnose the nuclear contents of sealed containers such as storage caskets. While gamma-ray imaging is an established means for gamma-ray detection in medicine and astrophysics, gamma-ray imaging for safeguards is still in its infancy. With the new Global Nuclear Energy Program gamma-ray imaging as a tool to verify materials 
in nuclear materials and to prevent diversion might become essential for the whole fuel cycle.

Finally, the potential impact of gamma-ray imaging for Homeland Security applications was realized only recently. One of the outstanding challenges for homeland security is the detection of nuclear threats (Radioactive Dispersal Devices or RDDs, Improvised Nuclear Devices or INDs, or nuclear components) at US borders, in transit to potential targets, or in place prior to terrorist attack. This challenge is due to the fact that many of the nuclear materials associated with potential nuclear threats may have weak radiation emissions. This challenge is compounded by the fact that any countermeasure must deal effectively with the many legitimate radioactive objects found commonly in commerce and in the environment. Current passive radiation detection schemes lack in sensitivity and specificity in uniquely detecting, identifying, and localizing special nuclear materials. This deficiency is multiplied in wide-area surveillance applications in which weak threat sources have to be distinguished from spatial background fluctuations and in the midst of legitimate radioactive sources. These limitations cause too many nuisance alarms, which cannot be mitigated without significant interference with commercial traffic and cause other operational deficiencies as well.

The above mentioned areas of applications cover the full range for gamma-ray imaging implementations and purposes: Gamma-ray imaging to measure the location and the extension or shapes of radioactive distributions, e.g. in nuclear medicine or for diagnostics of nuclear materials; Gamma-ray imaging to detect weak sources which could have otherwise not be detected. Then we can distinguish between near-field imaging, as in nuclear medicine with the object very close to the imager, typically in the order of 10$40 \mathrm{~cm}$ and far-field imaging, as for potential search and surveillance in homeland security, which aim on detecting weak sources up to $100 \mathrm{~m}$ distance. Finally, the field-ofview can be small, e.g. for diagnostics purposes in safeguards or astrophysics, or large, e.g. for surveillance, search, and monitoring.

In the following we discuss in more detail the ability to increase the sensitivity in detecting nuclear materials through gamma-ray imaging before we conclude this introductory chapter with some additional remarks.

\subsection{Relevance to Search and Surveillance Applications}

To address the problem of lack of sensitivity for large gamma-ray detectors the concept of gamma-ray imaging was proposed several years ago to make more efficient use of large systems [3]. Large detectors with imaging capabilities will enable large areas both on land and in air to be monitored. It is also the only way to survey inaccessible areas. How gamma-ray imaging can improve sensitivity by distinguishing between a localized signal and a distributed background is shown in Figure 2. A conventional, non-imaging instrument can measure the count rate which reflects the incident gamma-ray flux and the energy of the incident gamma rays. Without shielding or collimation it accepts gamma rays from all directions and can be overwhelmed by local background radiation. A gamma-ray imager can observe the gamma-ray flux and the gamma-ray energy as well, 
however, in addition, it can isolate sources from each other and from local background (at the expense of added complexity). An ideal gamma-ray imager can maintain the whole signal from the source while reducing the background down to the background at the source location. A gamma-ray imaging system can "count" on a pixel-by-pixel basis in which each pixel represents a part of the total solid angle. The signal of the potential threat source is limited to one or only a few pixels while the background is distributed over all pixels. The more pixels the system has the better the background can be effectively reduced. The signal-to-noise can be increased until the pixel size represents the extension of the source and its direct surrounding such as shielding.

A shielded source might emit a significant fraction of its total activity as down-scattered gamma rays. In some cases, these in-shield scattered gamma rays will represent the strongest signal from a source. While a conventional detector even with excellent energy resolution will not be able to identify the source and will have difficulty in recognizing such a source at all, a gamma-ray imaging system will still be able to recognize a compact source with high sensitivity. To demonstrate the gain in sensitivity by employing gamma-ray imaging we have performed Monte-Carlo simulations to determine Receiver Operator Curves (ROC) for different degrees of angular or imaging resolution.

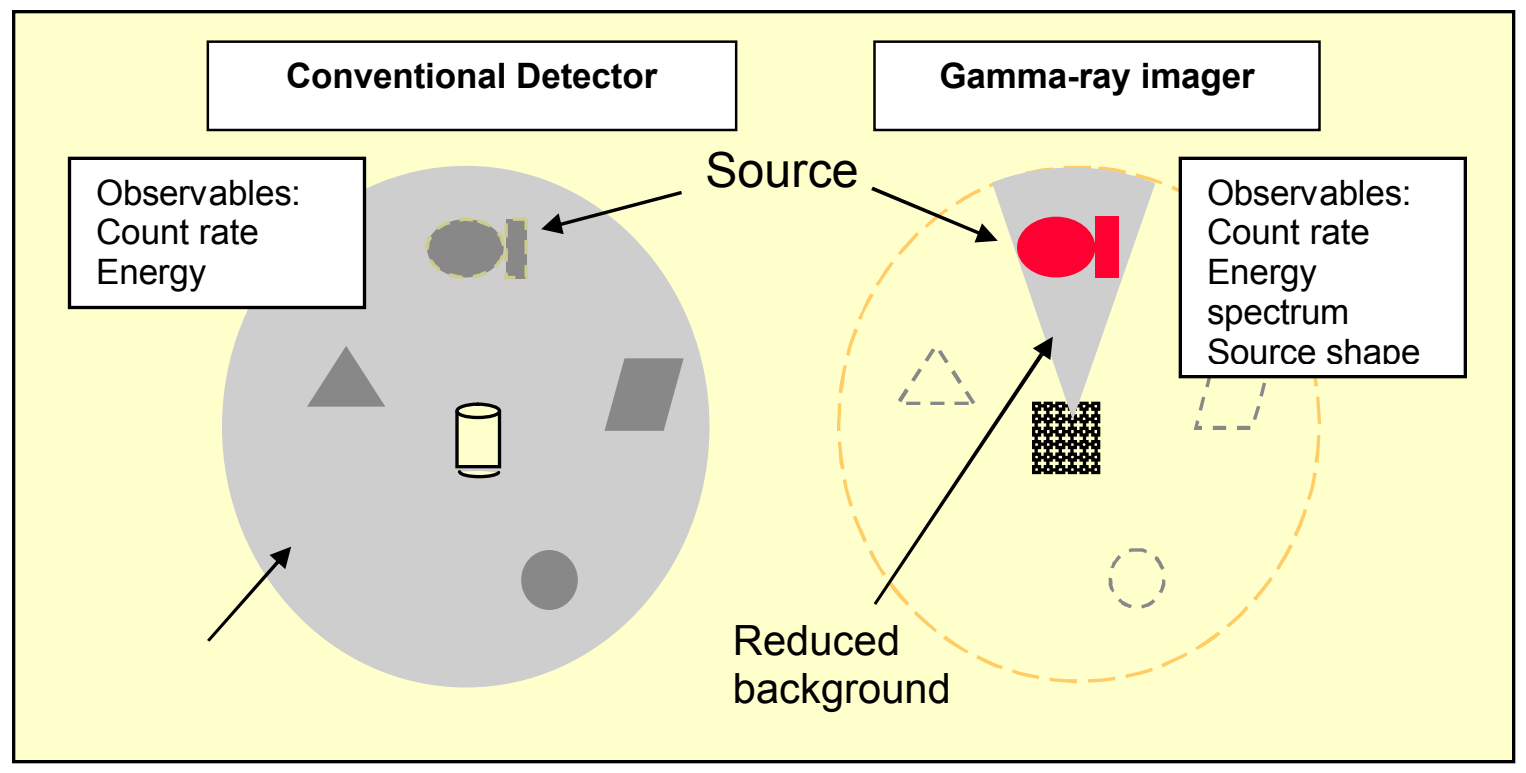

Figure 2: Schematic comparison between conventional, non-imaging (left) and imagingbased (right) gamma-ray detection. The conventional detector can observe the gammaray flux or count rate and the gamma-ray energy. Without shielding or collimation it accepts gamma rays from all directions and can be overwhelmed by local background radiation. A gamma-ray imager can observe the gamma-ray flux and the gamma-ray energy as well, however, in addition, it can isolate sources from each other and from local background. An ideal gamma-ray imager can maintain the whole signal from the source while reducing the background down to the background at the source location. 
Figure 3 shows the results of these calculations. Assumed here is a detector, which is mounted in the air $100 \mathrm{~m}$ above the ground with an area of $1 \mathrm{~m}^{2}$ and an efficiency of $100 \%$. Natural background is also assumed from the ground, which is emitting 2 photons $/ \mathrm{cm}^{2} / \mathrm{s}$, which represents a typical ground activity. The spectral composition of the background is taken from the Pohl parameterization [4]. All photons above an energy threshold of $90 \mathrm{keV}$ are accepted resulting in about 1.2 million photons per minute registered in the detector (or a count rate of about $20 \mathrm{kHz}$ ). The source is assumed to emit 500 counts per minute into the detector, which corresponds to a $300 \mu \mathrm{Ci}^{137} \mathrm{Cs}$ source or 2 $\mathrm{kg}$ of unshielded WGPu. Without imaging (equivalent to only one spatial bin expressed as angular resolution), and a measurement time of one minute, we observe about 20 false alarms per hour or one false alarm every three minutes or every third measurement for a detection efficiency of 50\%. Assuming we can subdivide the solid angle into 100 (10x10 array matrix) bins we can reduce the false positive rate to 0.1 false alarms per hour (red curve). For 200 bins we reduce the false positive rate to below $10^{-4}$ and even for a detection efficiency of $90 \%$ the false positive rate is now 0.1 per hour. For 400 bins we observe effectively no false positives even at $100 \%$ detection probability. This demonstrates the potential for a significant increase in the sensitivity by employing gamma-ray imaging with the goal to maximize the detection probability while minimizing the false alarm rate.

Figure 3: Impact of gamma-ray imaging on sensitivity in terms of ROC curves for different angular resolution (pixels). Shown are efficiencies of detection (true positives) vs. false alarms per hour (false positives) for different bins (pixels), which divide $4 \pi$.

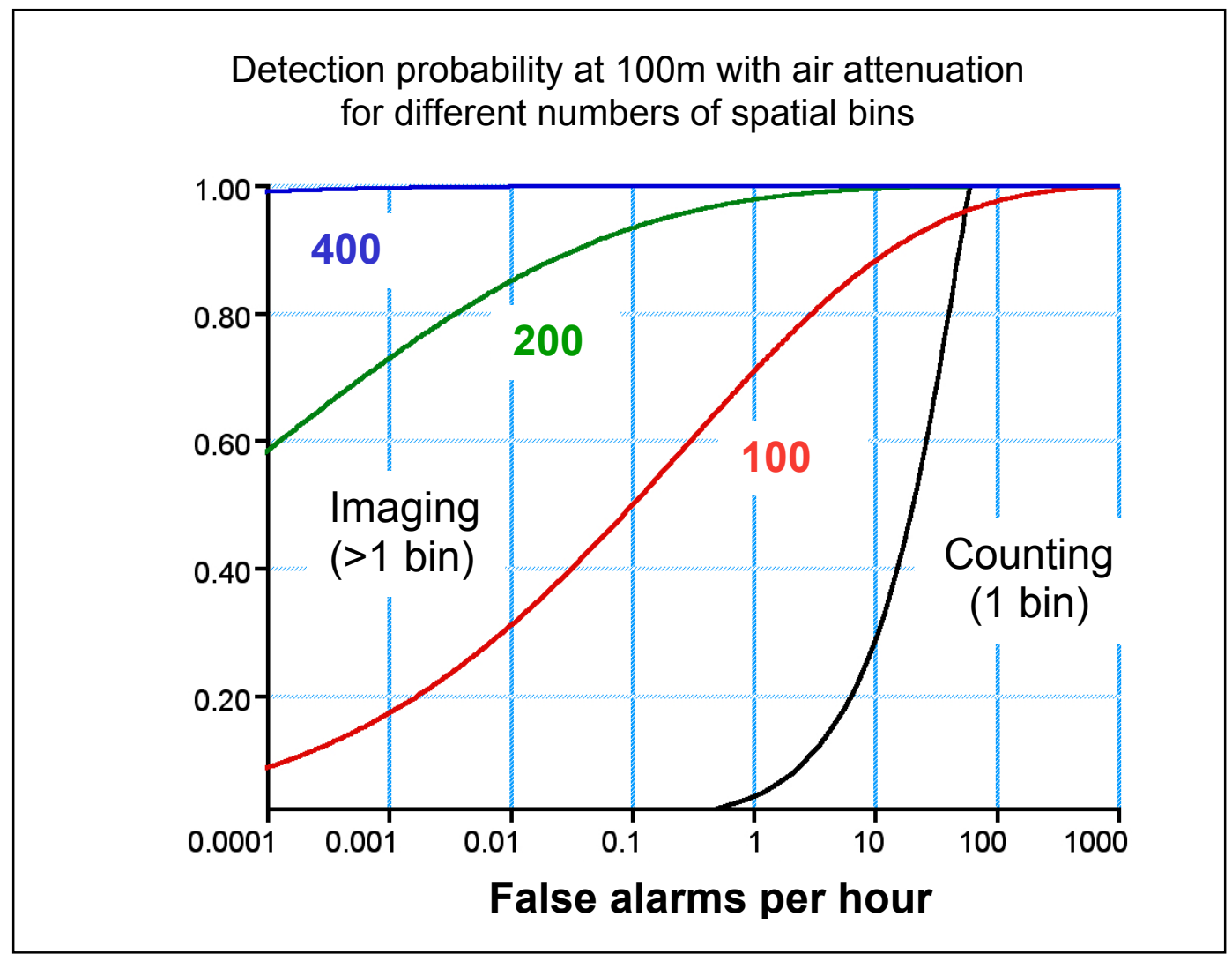




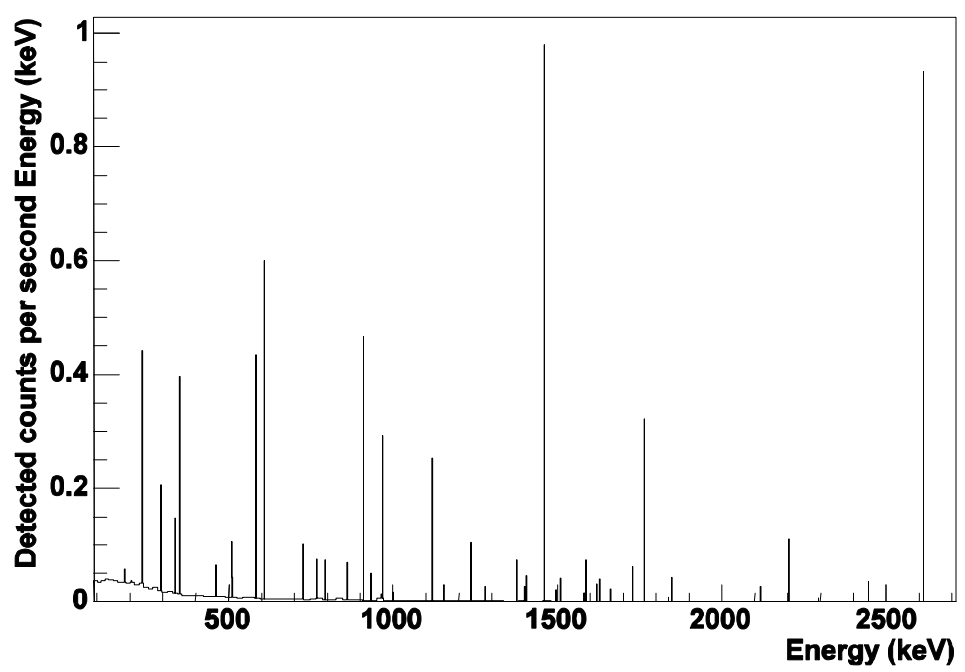

Figure 4: Calculated photon energy spectrum for the background assumed in the simulations.

In the previous calculations a Poisson distributed background distribution was chosen. For static scenarios (i.e. fixed detector and fixed source) this is a valid assumption. However, for dynamic scenarios like search and even portal monitors the background radiation does not follow the Poisson distribution but has a tail to higher count rates. This observation reflects the fact that significantly higher count rates than a Poisson statistic would allow are present in the natural background. Without the imaging capability, which allows us to identify the locally varying background, the false alarm rate in Figure 2 would be significantly worse. Figure 5 shows measured count rates obtained at 2950 locations around LLNL in Livermore with a $0.57 \mathrm{~m}^{2}$ detector. The average count rate observed was 8955 counts per second [5]. Assuming a Poisson distribution we would expect a spread of $\sqrt{8955}=95$. An alarm threshold at $5 \sigma$ results therefore in 9428 counts per second as shown as the red line in the left plot of Figure 5. However, the plot on the right shows the actual measured background count rates, which is much broader than expected and has a tail to higher count rates. Instead of 9428 the $5 \sigma$ threshold is actually at 14215 . However, many threat sources can potentially be missed if we take 14215 as our alarm threshold. Therefore, the imaging capability is crucial in lowering the alarm threshold and being able to identify the areas with increased count rates and associate them with natural background to mitigate the effectively false alarms. 

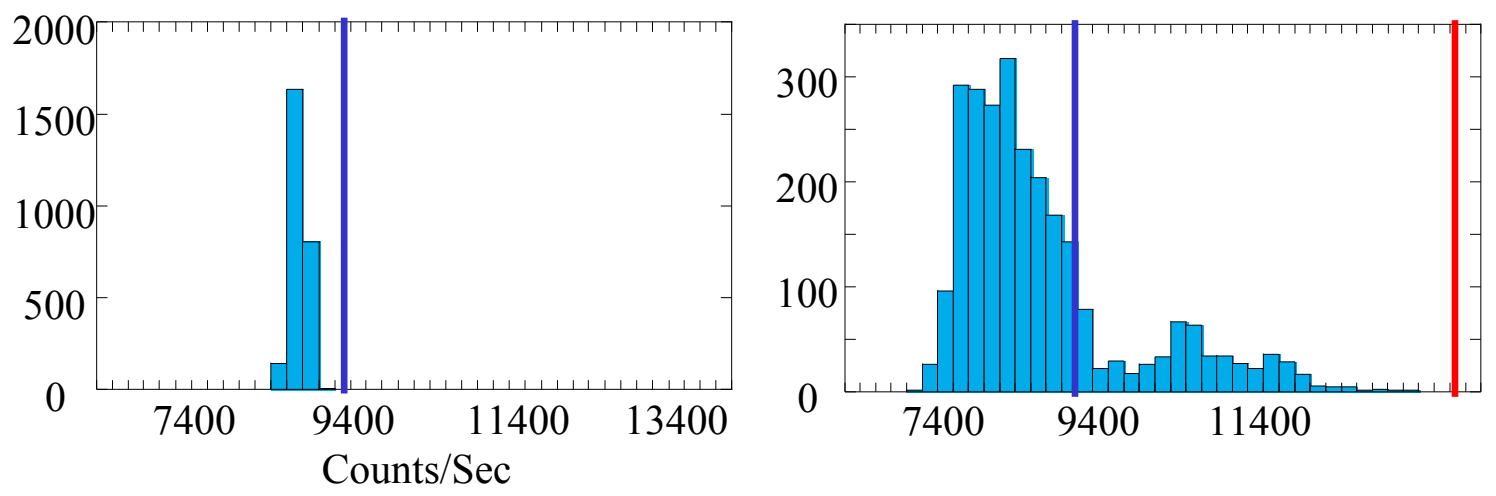

Figure 5: Expected (left) and measured (right) distributions of background count rates with $5 \sigma$ thresholds according to a Poisson distribution (blue vertical lines) and according to the real distribution (red vertical lines) (see report on Large Area Imager).

In addition to this increased sensitivity in detecting radioactive sources, which is crucial particularly for large detectors, which are otherwise overwhelmed from natural background and background fluctuations, an imaging system provides the source locations and potentially the extension of a source as well. The ability to localize the source is extremely important for follow-up operations where we want to know exactly where to look for the source. Here the merging of visual images with gamma-ray images is an essential tool to identify the object and to mitigate the alarm or to guide further investigation.

Furthermore, as in medical imaging, source shapes and extensions can be obtained. This has a significant implications e.g. in secondary inspection or follow-up search to distinguish between a compact a potentially dangerous source from an extended and very likely harmless source as can be found in bags of soil or fruits. In addition, as before, the attempt of terrorists to mask a weak threat source with a strong legitimate source can be prevented since both sources can be distinguished.

\subsection{Distinctions Among Imaging/Directional Detectors}

Before we discuss the concepts and possible implementation for gamma-ray imaging more specifically, it is necessary to add a few more general remarks on this topic. We want to point out that we distinguish between a gamma-ray imaging system and a gamma-ray detector, that provides some directionality. We define a gamma-ray imager as a system which is able to provide the location of a point source to better than $10 \mathrm{deg}$ and is able to deduce the extent or shape of a source. A directional gamma-ray detector is only able to provide the approximate location of a source with angular precision between $10 \mathrm{deg}$ and $60 \mathrm{deg}$. In the extreme case it can only determine the location to the side of the instrument. For some applications, directionality might be sufficient and it would be an improvement over instruments which are not able to provide any information about the location of a source. Although we call this study gamma-ray imaging study we do include direction-finding systems as well since a rough position estimate is sufficient and 
certainly better than no information about the location at all in many potential applications. In addition, directional systems will typically be much less complex than true imagers and therefore cheaper and easier to deploy. Many implementations can be envisioned for directional systems which are based on only modest modifications of systems which are already deployed such as $\mathrm{NaI}(\mathrm{Tl})$ based systems. These modifications range from assembling several detectors in certain patterns or by adding collimators to the system to obtain directional information.

Finally, we need to point out that we also distinguish two more classes of imagers: stationary and moving. A stationary system provides imaging without the requirement of a moving source or a moving detection system. However, systems can be built that provide imaging information only when in motion relative to a source of interest. The simplest "scanning" system of this type is of course a simple standard detector, which is being moved across a source and localizes the sources by the increased, potentially energy-specific count rates when being closest to the source. This simple proximity imaging works fairly well for small and handheld systems to find near-field sources. However, it fails when the background is more complex, particularly for larger distances (typically at detector-to-source distances larger than $10 \mathrm{~m}$ ). In addition, proximity imaging requires a controlled movement normally achieved by a human operator. However, in some applications, the risk for a human operator to receive a high radiation dose while mapping out activity levels might become too high and true imagers are required. For larger standoff distances which require larger detectors to compensate for the loss in solid angle, a simple scanning system has only a very limited sensitivity to the location of a source due to: 1.) The higher background rate due to the increased size of the detection system and 2.) A priori unknown temporal and spatial background fluctuations, which can "fake" a real source. With regard to the first point, one has to realize that the average background rate from the ground is about $20 \mathrm{kHz}$ per $\mathrm{m}^{2}$, which implies that any detector, which is in the order of $0.1-1 \mathrm{~m}^{2}$ will have $1000 \mathrm{~s}$ of counts per second just due to natural background, if no shielding from the ground is provided. With regard to the second point, as discussed before, temporal and spatial fluctuation in the background requires high alarming thresholds to reduce false alarms, and unfortunately also reduces the sensitivity to detecting a source significantly.

However, instead of relying exclusively on count rates to deduce the location of a source, collimation and attenuation systems can be added to the standard detectors to reduce the field-of-view of what the detector can "see". In this way, the system counts only the local background and the local signal, which is defined by the extension of the FOV. As with the unshielded systems discussed before, only due to the - most likely one-dimensional movement of the detector it is possible to map out the local activity levels, which passed by. A moving coded aperture system as currently being demonstrated by LLNL is a very advanced implementation of such as system [5]. 


\subsection{Cramer-Rao lower bound estimates}

To compare imaging sensitivities between various gamma-ray imaging systems independently of particular image reconstruction techniques, the Uniform Cramer-Rao (UCR) lower-bound estimate can be employed [6,7]. The UCR lower bound can be applied to predict the limiting performance of gamma-ray imaging systems or used as a figure of merit to optimize system design. It is particularly useful for a study such as this one which aims at the comparison of very different imaging techniques for a specific application. Due to the complexity of this evaluation we are not able to perform these calculations within the scope of this report, however, we will describe this particular too in this section. We are planning to implement the UCR lower bound estimates in the near future and perform quantitative comparisons for specific scenarios.

UCR lower bound is a mathematical tool developed to provide a lower bound on the variance of a pixel of interest dependent on the system and data statistics alone, and not upon any particular estimator. For a pixel of interest, the variance will be a function of the pixel intensity bias gradient length. The bias-gradient length can be interpreted with caution as a measure of the width of the point spread function. The bias-variance tradeoff curve will indicate the achievable region of the system (see Figure 6). An ideal system would provide both a small variance and bias. Therefore, UCR bound curves allow comparisons between imaging systems which operate under widely different principles, and the comparisons which are made are valid irrespective of reconstruction algorithm.

The rationale behind using Cramer-Rao lower bounds comes from communication theory, where information loss is usually expressed in terms of the amount of errors introduced during data transmission through noisy channels. For gamma-ray imagers, the variance of a reconstructed pixel intensity can increase due to noise propagation from other pixels during the decoding process. This information loss is known as "decoding penalty", and is defined as the ratio of variance of the reconstructed intensity for a pixel of interest for two competing gamma-ray imagers

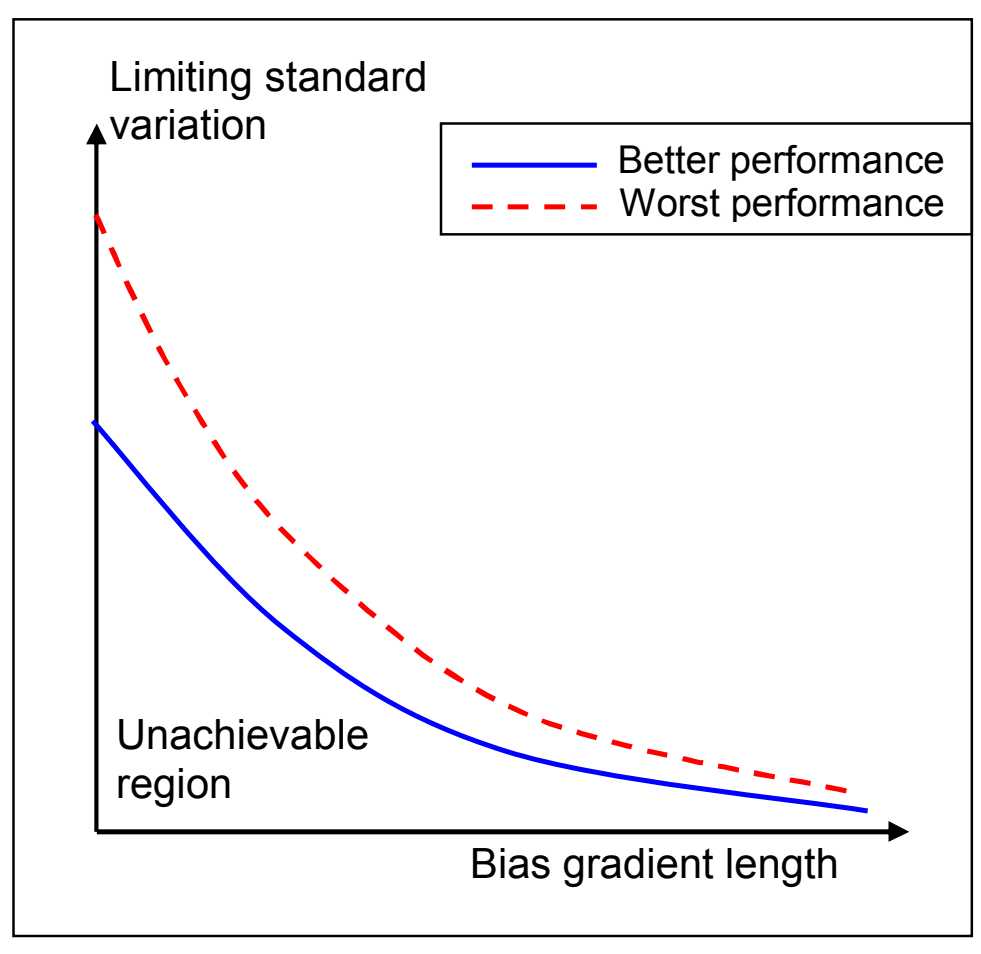

Figure 6: Bias-Variation trade-off curves determined by Cramer-Rao lower bound estimates indicate the limiting performance of an imaging system for a given source model. 
on a per-detected-photon basis.

The higher the decoding penalty, the larger the information loss will be. UCR lower bound provides an quantitative tool to calculate the decoding penalty.

Steps for calculating UCR bounds:

- Assume a source scenario.

- Model the response of all considered gamma-ray imagers for the assumed source. Calculate system matrix $A$

- Calculate the Fisher information matrix $F=F(A)$

- For $\lambda \in(0, \infty)$ calculate the inverse matrix $M=(I+\lambda F)^{-1}$

- Determine the bias gradient $g=g(M)$ and variance $\sigma=\sigma(M)$

The calculation of the lower bound is based only on an accurate system model at a finite parameterization of the source. One of the problems with bias is that it is very object dependent. When increasing the regularization parameter, the bias does not always monotonically increase, but depends on what structures are being smeared into the parameter of interest.

By properly evaluating the bias gradient image generated when simulating or measuring the UCR's bound, it is possible to generate a mapping of each system's bias gradient length to the more familiar FWHM measure of image position resolution. Lower bound results can be scaled to a per-detected-photon basis which corresponds to a figure-ofmerit for the relative information that is carried by a single detected and desired event. In this way, a decoding penalty factor can be derived. Thus, the decoding penalty gives information about how many more photons need to be collected in a certain imaging system to achieve the same sensitivity (e.g. equivalent amount of information) as another imaging system.

As an example, it was shown that the enhanced Compton-imaging based C-SPRINT system [8] matches the performance of the parallel-hole system at $140 \mathrm{keV}\left({ }^{99 \mathrm{~m}} \mathrm{Tc}\right)$, and outperforms the parallel-hole system at $364 \mathrm{keV}\left({ }^{131} \mathrm{I}\right)$. The authors of these papers conclude that the sensitivity of this still not optimized system at this or higher energies can be increased significantly over the collimator-based system. 


\section{Overview of Gamma-Ray Imaging Concepts}

In this chapter we provide a brief overview of imaging concepts. Many of these are discussed in more detail in the Appendix.

As previously discussed, the ability to image distributions of gamma-ray sources is well established especially in the medical field, e.g. to diagnose and treat cancer using radiolabeled targeting agents or to develop new drugs and pharmaceuticals.

The widely used imaging systems consist typically of collimators in front of positionsensitive NaI(Tl) detectors. These - Anger camera - systems are inherently limited by the trade-offs due to the collimator which limits the geometric efficiency to $\sim 10^{-4}$ for maximum gamma-ray energies of $400 \mathrm{keV}\left(364 \mathrm{keV}\right.$ of $\left.{ }^{131} \mathrm{I}\right)$. As an alternative to these multi-hole collimators, pinhole collimators are used as well which can provide very high spatial resolution particularly for near-field applications such as small animal imaging. However, efficiencies of pinhole collimators are typically $10^{-6}$. In addition to the collimator-based trade-off between spatial resolution and geometrical efficiency, several factors degrade the performance and the usefulness of these systems, particularly for higher gamma-ray energies $(>400 \mathrm{keV})$. Here, the penetration through the collimator or the shields, the scattering in the collimator and the scattering in the detector significantly limit the performance of any collimator system. Furthermore, standard multi-hole collimators are generally limited for larger standoff distances when the angular resolution and the field-of-view are the same and imaging is not possible anymore (since no structure can be resolved within the FOV). Here, a simple tube collimator is more sensitive than a parallel-hole collimator and imaging can only be achieved when operated as a scanner with a moving source or a moving instrument. A pinhole collimator on the other hand can still be used as a true gamma-ray imager for larger standoff distances and the FOV and spatial resolution can be adjusted by changing the focal length, e.g. by changing the distance between the collimator and the position-sensitive detector. However, the efficiency will be extremely low since the hole needs to be significantly smaller than the detector area to achieve imaging capability within the FOV.

To increase the efficiency and the FOV, more advanced collimators such as multi-pinhole or coded aperture (CA) systems have been developed. While multi-pinhole systems are only being used for near field such as small animal imaging applications, coded aperture systems have been built recently for near- and far-field applications [5]. Coded aperture systems rely on a collimator aperture pattern that uniquely modulates each position in the FOV onto the position-sensitive gamma-ray detector. The transmission efficiency can be increased to $50 \%$, which is significantly higher than the transmission efficiencies in pinhole or parallel-hole collimator systems. Envisioning a coded aperture with $N$ holes instead of one for a pinhole, the signal-to-noise is increased by $\sqrt{N}$. Similarly to the modulation of a coded aperture in space, the modulation of a source location can be performed in the time domain, too. These systems are called rotation modulator (RM) and rotation modulation collimators (RMC). In a rotation modulator, a position-sensitive detector itself is rotating and therefore modulates the source location. In a rotation modulation collimator, typically two slit collimators are rotated in front of a non-position sensitive gamma ray detector. The advantage of a RMC is that no position sensitive 
detector is required for imaging and standard detectors, which are already built and deployed can be converted into RMC imagers. For instance, one can envision to add a RMC to a mechanically cooled HPGe detector to provide not only high efficiency and high energy resolution of a HPGe system but also imaging. Potentially, one can add different types of RMCs to match the needs for a variety of operations. The drawback of a RMC system is the limited FOV, which allows the implementation for diagnostics purposes where the location of the object is known and the extension fits into the FOV but it prevents the implementation as search and surveillance instrument.

Such modulation systems can be implemented as true and stationary imagers, e.g. for diagnostics or monitoring purposes and they can be mounted on moving platforms where the imaging can be used to separate a signal from spatial and temporal background fluctuations.

While the efficiency of these configurations can be increased significantly over the conventional parallel-hole systems, the imaging efficiency is limited in high activity and complex backgrounds. In the worst case, a coded aperture is equivalent to a simple pinhole system. In addition, the modulation systems have the same drawback as the parallel hole system with regard to the weight particularly if gamma rays with energies up to $3 \mathrm{MeV}$ are to be imaged.

For many applications, one would like to build a gamma-ray imager without a collimator or aperture. Not only would it allow us to build a much lighter and more compact system but also not having a collimator would allow for an increased detection efficiency. In fact, collimator-based systems reduce the flux from the source due to the collimator or the aperture while the background can enter the detector without reduction if no shield is provided. However, providing a shield around the collimator-based system will increase the weight even more. Even an optical gamma-ray system which would be the ideal for imaging purposes would benefit from shielding as would a Compton imager (to be discussed below).

Two collimator-less gamma-ray imager concepts are a gamma ray lens and a Compton imager. The - in terms of imaging - ideal gamma-ray imager, a gamma-ray lens, is very difficult to realize due to the small angle of total reflection for gamma rays. Multilayer, diffractive optics systems have been built which are able to focus gamma rays up to 160 $\mathrm{keV}$, however, these systems are characterized by a large focal length and very small field-of-view [9]. The FOV is typically less than $1 \mathrm{deg}$. and the focal length is in the order of several meters. In addition, for higher gamma-ray energies, multiple interactions in the position-sensitive detector degrade the performance, as in the collimator-based systems. Also as in the collimator-based systems, gamma rays from outside the FOV cannot be separated except when shielding is used. A few prototype systems are currently being built for photon energies below $100 \mathrm{keV}$ for small animal studies [10] and astrophysics applications [11]. Due to the limitations in terms of gamma-ray energies, FOV, and size, gamma-ray focusing optics imagers are not considered further in this report. 
An alternative way to image gamma rays without the use of a collimator is Compton imaging [12-22]. This imaging concept relies on the Compton effect and therefore converts the otherwise limiting process of multiple interaction in the detector into a required process. While the concept was developed about 40 years ago [23,24], only recent advances in the two-dimensional segmentation of semiconductor detectors along with signal processing allow us now to build efficient and high-resolution Compton imaging systems. The first instrument which was built and successfully demonstrated was the Comptel instrument which was a space-based gamma-ray observatory performing measurements in the 90s [12]. While many new features were discovered in the energy range between $500 \mathrm{keV}$ and several $\mathrm{MeV}$, it was realized that the overall sensitivity was very limited, mainly due to the underlying technology of large-volume, position sensitive $\mathrm{NaI}(\mathrm{Tl})$ detectors which were separated by several meters to achieve reasonably high angular resolution. In fact, the two $\mathrm{NaI}(\mathrm{Tl})$ layers were so far separated that the time resolution of several ns of the detectors were sufficient to time order the interactions. State-of-the art, two-dimensional position-sensitive semiconductor detectors along with pulse-shape processing allows us to circumvent most of the limitations of Comptel and increase the sensitivity significantly. In contrast to collimator or modulation-based gamma-ray imaging instruments, a Compton imaging systems is the only one which is truly able to distinguish between background and a source since it is able to measure both simultaneously. For instance, a coded aperture system can only reduce the intensity of the background over the signal but cannot distinguish them completely. In addition, since no heavy collimator or aperture is required, compact and relatively light-weight systems can be built, which is important for a range of applications, particularly for air-deployed platforms. For instance, a large-area Compton imager with $1 \mathrm{~m}^{2}$ effective area can be envisioned with a weight of the order of $1000 \mathrm{lbs}$, in contrast to the recently demonstrated large-area coded aperture system which weighs about $4000 \mathrm{lbs}$ for an effective are of $0.57 \mathrm{~m}^{2}$ [5]. The drawback of a Compton imaging is its complexity and potentially the cost. This is due to the fact that high three-dimensional position and high energy resolution is required to measure individual gamma-ray interactions in the detection volume. The detection material and the instrument geometry has to be chosen carefully to be able to induce multiple interactions and to be able to measure the angle between the interactions with high accuracy and high efficiency. Many Compton imaging implementations can be envisioned, ranging from a variety of gases with different pressures and readouts to a variety of different semiconductor materials, with different segmentation and readout schemes. However, beyond the determination of the positions and energies of the individual gamma-ray interactions, all systems require the same processing steps, ranging from properly time-ordering or "tracking" of the interactions to image reconstruction, correlation analysis, and decision making. 


\section{Technology Summary Table}

Here we present a summary of gamma-ray imaging technologies and their associated characteristics in terms of performance and engineering parameters. We divide the large range of possible concepts and implementations of gamma-ray imaging systems in 5 categories reflecting fundamentally different approaches:

1. Directional imagers

2. Collimator-based imagers

3. Coded aperture imagers

4. Rotation modulation imagers

5. Compton imagers

The following table summarizes basic performance and engineering parameters for the five identified categories of gamma-ray imaging methods. As discussed in more detail in the appendix of the methods section, the five categories consist of directional, simple collimator-based, coded aperture, rotation modulation, and Compton imaging. The directional imagers are simple devices which consist of non-position sensitive detectors which can determine the location of sources just by proximity imaging which requires at least the detection system or the source to be moving. These systems can either be simple and standard, omni-directional detector, they can be shielded either by passive or by active means. Shielding can provide a shadow which can be used to deduce the source location more accurately and reliably than possible with an unshielded detector. Collimator imagers use not only shielding but active collimation to deduce the location of sources. The classical example is the pin-hole imager which can be used in stationary and mobile applications. Another example is a tube-like collimator which requires a relative movement again to provide imaging capabilities. The tube-like imager is the extension of the parallel-hole imager for large stand-off distances. The coded aperture system decodes the incident gamma-ray flux spatially, either in one or in two dimensions. It has a fairly high aperture transmission efficiency, however, degrades in performance in more complex and higher-level radiation fields. The rotation modulation imager decodes the gamma-ray flux temporarily. In contrast to the coded aperture system, no positionsensitive gamma-ray detector is required. This allows us to use standard detectors which are deployed already to be converted to imaging detectors by adding the rotating mask. While the angular resolution can be really high, these systems are limited by the FOV which can be observed. The last category is the Compton imager which is able to provide true gamma-ray imaging capabilities with a large FOV without using a heavy collimator. Such a system is superior to modulating systems for complex and high radiation fields, however, they are more complex and more expensive since they require high-position and high-energy resolution detection systems. Many Compton imaging configurations can be envisioned are currently being explored, ranging from single to arrays of detectors and cryogenically cooled HPGe to room-temperature operated CdZnTe and Si detectors. While several Compton imaging concepts are currently being developed and demonstrated it will likely take at least two more years to see Compton imaging systems deployed in the field. 
Table 1: Summary of performance and engineering parameters of the five gamma-ray imaging categories.

\begin{tabular}{|c|c|c|c|c|c|c|}
\hline Parameters & Directional & Collimator & Coded & \multicolumn{2}{|c|}{ Rotation Modulation } & Compton \\
\hline $\begin{array}{l}\text { Imaging } \\
\text { dimensions }\end{array}$ & 1 & 1 or 2 & 1 or 2 & 1 & 2 & 2 (or 3) \\
\hline FOV [deg] & $30-360$ & $<60$ & $<60$ & 360 & $<60$ & 180 or 360 \\
\hline $\begin{array}{l}\text { Angular } \\
\text { Resolution [deg] }\end{array}$ & $>30$ & $<30$ & $<5$ & $>10$ & $<5$ & $1-5$ \\
\hline $\begin{array}{l}\text { Energy } \\
\text { Resolution }^{1}\end{array}$ & $\begin{array}{l}\text { Very Poor - } \\
\text { Excellent }\end{array}$ & $\begin{array}{l}\text { Very Poor - } \\
\text { Excellent }\end{array}$ & $\begin{array}{l}\text { Very Poor - } \\
\text { Excellent }\end{array}$ & $\begin{array}{l}\text { Very Poor - } \\
\text { Excellent }\end{array}$ & $\begin{array}{l}\text { Very Poor - } \\
\text { Excellent }\end{array}$ & $\begin{array}{l}\text { Good - } \\
\text { Excellent }\end{array}$ \\
\hline Imaging Efficiency & Poor & Very Poor & Good & Good & Good & Good \\
\hline Contrast & Poor & Excellent & Good & Good & Very Good & Very Good \\
\hline $\begin{array}{l}\text { Minimum Weight }{ }^{2} \\
(\mathrm{Kg})\end{array}$ & 0.1 & 20 & 20 & 20 & 20 & 0.5 \\
\hline Weight-Ratio $^{3}$ & $10-90 \%$ & $<<10 \%$ & $<10 \%$ & $<10 \%$ & $<10 \%$ & $>50 \%$ \\
\hline Achievable Scale & Large & $\begin{array}{l}\text { Medium - } \\
\text { Large }\end{array}$ & Large & Small & Small & $\begin{array}{l}\text { Medium - } \\
\text { Large }\end{array}$ \\
\hline Cost & Low & $\begin{array}{l}\text { Low - } \\
\text { Medium }\end{array}$ & Medium & Low & $\begin{array}{l}\text { Low - } \\
\text { Medium }\end{array}$ & $\begin{array}{l}\text { Medium - } \\
\text { High }\end{array}$ \\
\hline
\end{tabular}

\footnotetext{
${ }^{1}$ The energy resolution depends on the type of used detector. For instance, a rotation modulation system can be built with a high-resolution HPGe or low-resolution $\mathrm{NaI}(\mathrm{Tl})$ detector.

${ }^{2}$ Minimum weight is energy dependent. To have reasonable efficiency at high energy requires more material. Numbers given in table correspond to detectors relevant for $\sim 500 \mathrm{MeV}$ gamma ray energies.

${ }^{3}$ The weight ratio reflects the weight of the sensitive detector volume to the total weight of the instrument. For collimator-based systems, the total weight is dominated by the collimator and potentially required shields. For room-temperature operated Compton imagers the weight ratio can be increased to $>70 \%$. Cryogenically cooled systems require cooling with associated increase in weight and power. The exact ratio will depend on the platform and other potential constraint. The numbers quoted here reflect potentially achievable ratios.
} 


\section{APPEndiX: Gamma-RAY IMAgIng CONCEPT DETAILS}

In this part of the appendix we discuss possible imaging concepts and implementations more in detail. We distinguish between directionality and true gamma-ray imagers.

\section{Directionality Imagers}

In this section we will briefly discuss detection concepts, which provide some directional information about the source location, primarily by moving the gamma-ray detection system over a stationary source. This so-called proximity imaging is performed by counting the number of gamma rays as a function of time and/or location. It relies on the fact that the count rate in a detector depends strongly on the distance to the source. This concept is already well established in search applications, either in near field with handheld detectors or far-field, with large and airplane-mounted detection systems.

Time [s]

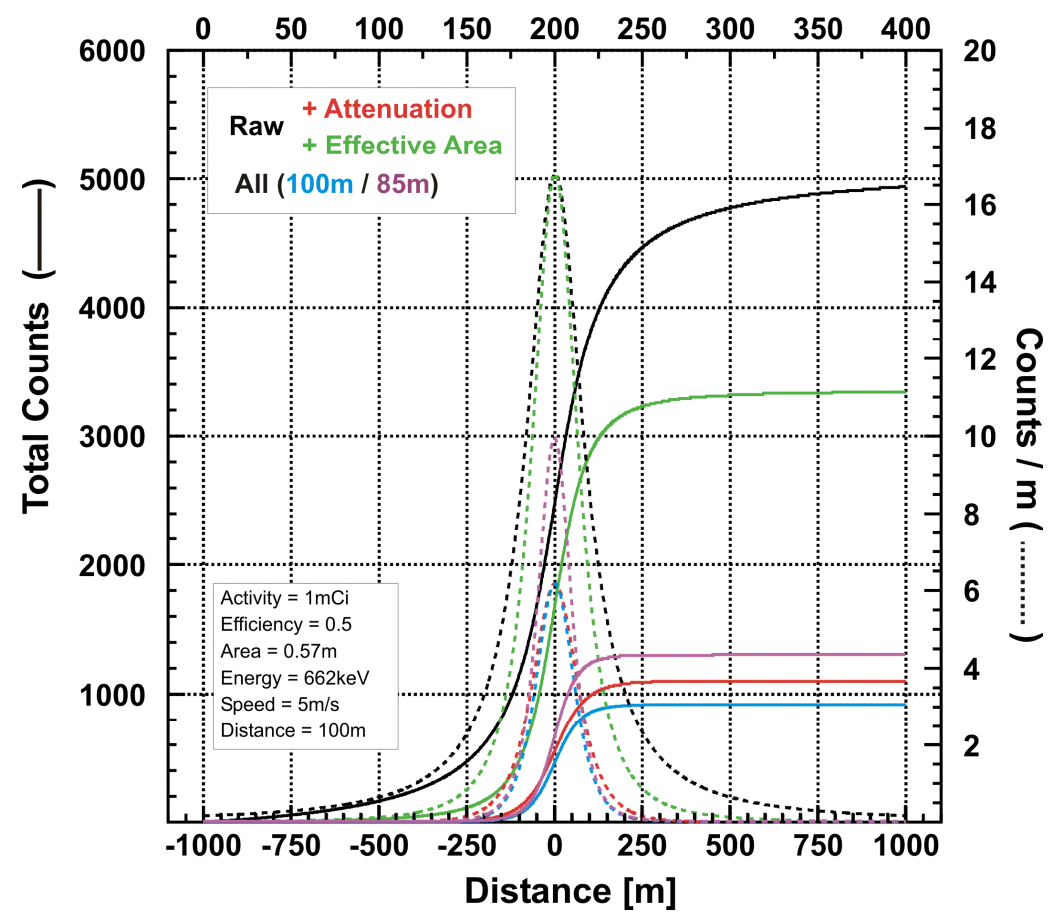

Figure 7: Calculated total counts (solid line) and count rates (dashed line) in a gamma-ray detector as function of position. The box on the lower left shows the parameters assumed for the calculations. The black lines represent counts without taking into account the effective area and attenuation of the gamma ray in air. The green and red lines indicate the count rates by taking the effective area and the attenuation into account, respectively.

Figure 7 illustrates proximity imaging by showing calculated counts and count rates in a gamma-ray detectors as a function of the distance to the closest location, which is $100 \mathrm{~m}$ away from the source in this case. Particularly by taking the attenuation and the effective 
solid angle as a function of distance into account, the source can be located by the increased count rate close to the nearest distance with an accuracy of about $150 \mathrm{~m}$ (FWHM). This plot also shows the number of total counts for the particular assumption indicated in the figure of 1100 and a maximum count rate of about 4 counts per meter or 20 counts per second since the speed is assumed to be $5 \mathrm{~m} / \mathrm{s}$.

This concept is already an established tool for airborne gamma ray surveillance in mapping and monitoring regional and local radiation levels, e.g. after radiological accidents, to study radiation biology, to perform geological mapping, or to prospect raw materials. Figure 8 shows an example of Skyvan, which is an aircraft of the Canadian Geological Surveillance Canada with 14 large volume NaI detectors with a total volume of 501 on board [25]. Figure 9 shows an example of the air surveillance concept with an aircraft flying at $80 \mathrm{~m}$ altitude with a speed of $60 \mathrm{~m} / \mathrm{s}$. As can be seen from Figure 7, the accuracy in the determination of the source location is limited by the distance of the detection system. In addition, the overall sensitivity is limited by the speed of the airplane, which reflects the trade off between count rate and total area to be covered. This concept was demonstrated to be useful when large areas in the orders of 10th of square miles need to be monitored or searched without very high resolution.
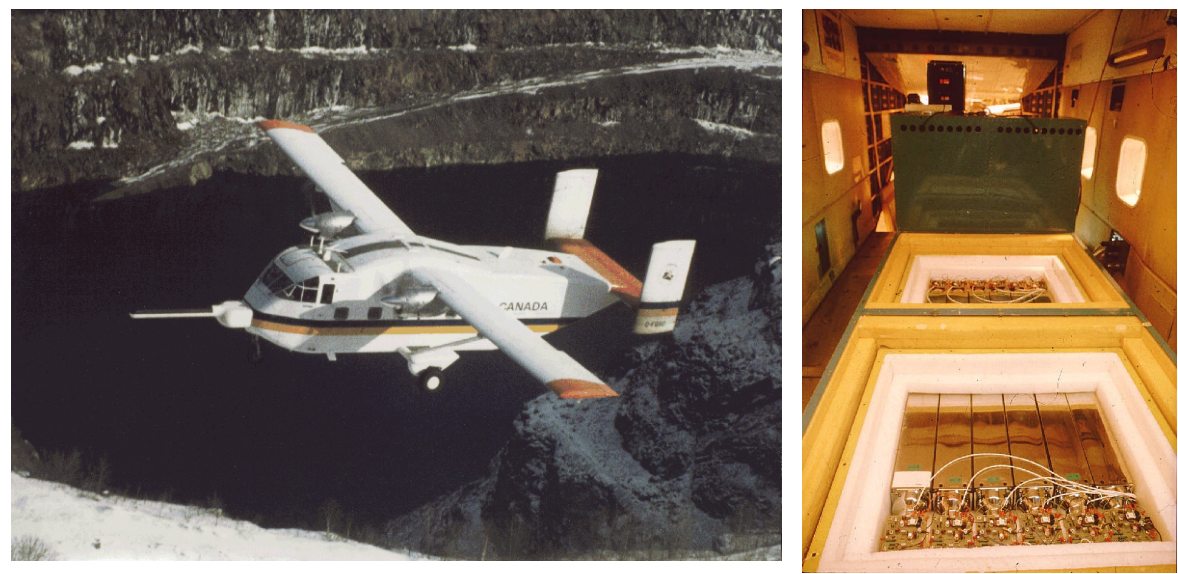

Figure 8: The Canadian Skyvan of the Canadian Geological Surveillance Canada (left) with 14 large volume $\mathrm{NaI}$ detectors with a total volume of 501 on board (right).

Similar techniques are used frequently with any kind of hand-held detector to localize sources in closed container, in rooms, etc. Overall count rates but in particular energyspecific count rates can be used to precisely localize sources when access and sufficient time for an operator is provided.

However, in many circumstances this is not possible. An example is the ARAM system, which was developed at LLNL and is now being used on chase vehicles to search and identify radioactive sources in urban and suburban areas [26]. It was realized that a single detection system does not provide sufficient information with regard to source locations and the usefulness is therefore limited. However, several simple schemes are being evaluated to at least allow to identify the side of the source. This can be accomplished, 
e.g. with one detector on each side with sufficient separation that the count rates will be sufficiently different depending on the side of the source.

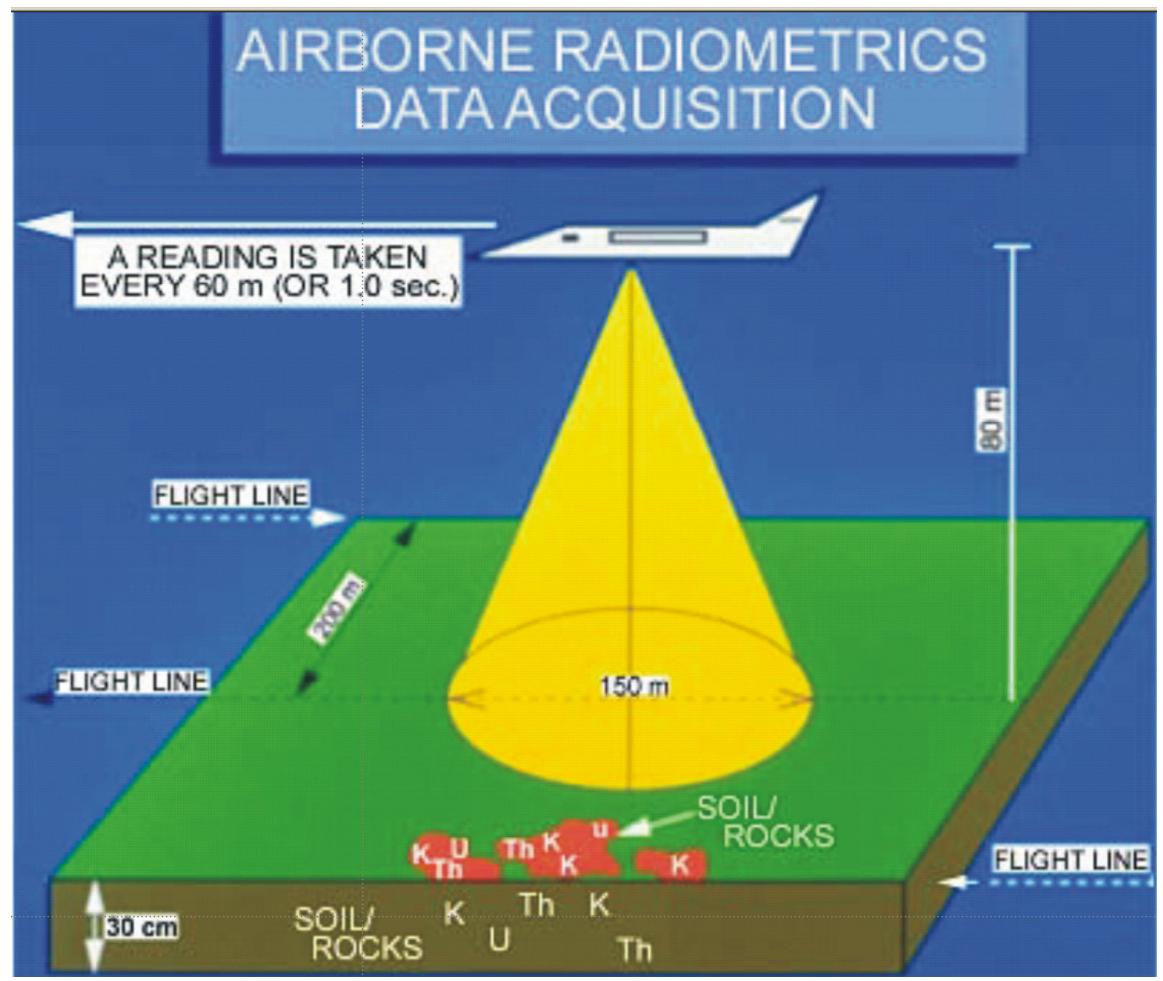

Figure 9: Principle of air surveillance concept with simple gamma-ray detector (taken from [25]).

A potentially better scheme is illustrated on the left of Figure 10. Two detectors are assembled close to each other and each detector serves as a shield for the other. In this way, one compact system can be deployed which is able to distinguish between left and right.
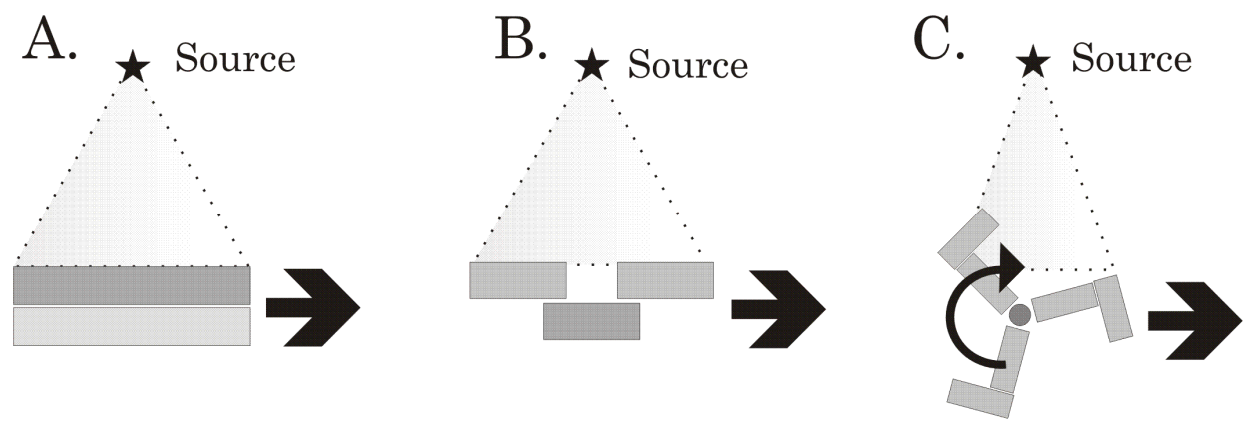

Figure 10: Simple detector configurations to provide rudimentary directionality information by self or active shielding without using a collimator. 
Figure 10 shows two more examples of self-shielding schemes, which can increase the resolution when moving relative to the source. Again, intensity ratios of all detectors provide directional information when coupled to the location of the system. Such an directional imaging scheme can also be used stationary when the system is rotating. Using appropriate filtering algorithms the temporary intensity pattern either due to translational or rotational motion can be unfolded into a spatial intensity distribution with an angular resolution of about $60 \mathrm{deg}$. These systems can be built of large and commercially available detectors such as $\mathrm{NaI}(\mathrm{Tl})$ scintillators. The weight and size of such systems will be mainly determined by the detectors and their specific configurations and layouts. However, on top of standard processing procedures of already deployed instruments - e.g. total and energy-specific count rates - "directional" information needs to be processed by correlating intensities, times, and positions.
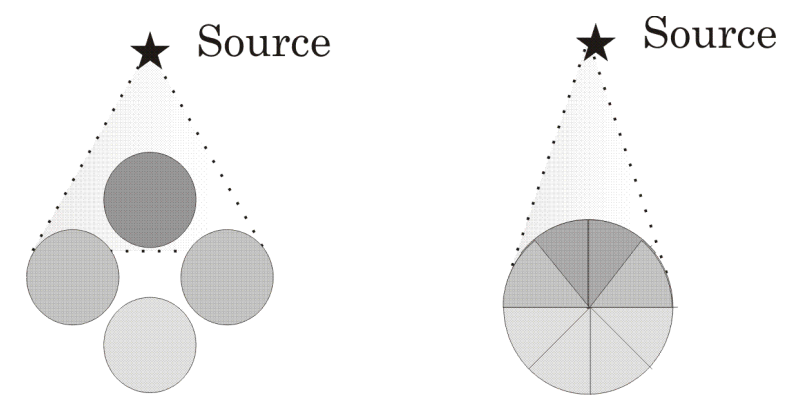

Figure 11: Possible configurations of cylindrical detectors such as coaxial Ge detectors to provide directionality.

Similar to these self-shielding concepts of potentially large detectors, e.g. built of $\mathrm{NaI}(\mathrm{Tl})$ or other scintillator materials, an array of coaxial Ge detectors or a single but segmented Ge detector can be used as a directional detector as well as shown in Figure 11. On the left a small array of coaxial detectors is arranged to provide directional information from $2 \pi$ through the attenuation of opposite detectors. On the right the same concept can be employed with a single but azimuthally segmented coaxial Ge detector. With such arrangements an angular resolution of about $60 \mathrm{deg}$. can be achieved as well, however, worse for higher gamma rays (e.g. above $1 \mathrm{MeV}$ ), particularly for a single detector since one segment is not able to attenuate the gamma ray flux sufficiently. The advantage of such a system is obviously much improved energy resolution of a Ge detector over the scintillator. However, the drawback is the operation of a Ge detector, which needs to be operated at liquid Nitrogen (LN) temperatures and the costs of Ge detectors.

In summary, directional gamma-ray detectors can be built easily by arranging state-of-the art scintillator or semi-conductor detectors and using simple algorithms to convert measured intensity ratios into a spatial distribution of gamma-ray sources. However, without the use of more refined passive or active collimation and shielding schemes these approaches are limited to about 60 deg angular resolution. 


\section{True Gamma-Ray Imagers}

In this section we discuss true gamma-ray imaging concepts and associated implementations in more detail. We begin with conventional, classical and collimatorbased imaging systems such as the pinhole or the parallel-hole systems. We then discuss more advanced modulation schemes such as the coded aperture and the rotationmodulation schemes before we discuss Compton imaging as a way to image gamma rays without collimators and modulation.

\subsection{Conventional, collimator-based systems}

In the following we will start the discussion with the simplest, collimator-based imaging system, the pinhole imager. While a pinhole imager can be built to any dimensions for any distances, a parallel-hole system is only useful for near-field applications and loses it imaging capabilities for larger distances, e.g. beyond $10 \mathrm{~m}$ in any reasonable collimator configuration. For larger stand-off distances the parallel-hole collimator acts only as a tube-collimator since each parallel hole sees all parts of the FOV.

\subsubsection{Pinhole imager}

The pinhole system is identical to the camera obscura in the visible domain and represents the simplest optical system to image an object onto an image plan. Figure 12 illustrates the concept.

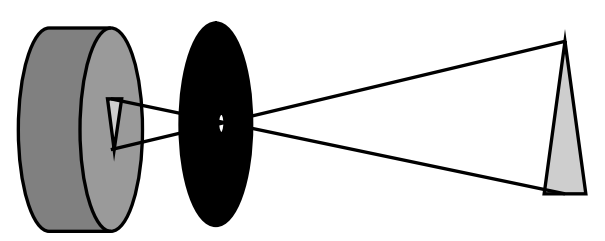

Figure 12: Simple illustration of a pinhole imaging system.

Each point of an object is projected uniquely through the pinhole onto the image plan. To image, a position-sensitive detector needs to be located at the imaging plan. Depending on the distance $B$ between collimator and object and the focal length $L$ (distance between detector and pinhole), the object is either magnified $(B / L<1)$ or reduced $(B / L>1)$ (see Figure 13). For the latter case, $B$ can be made large for larger stand-off detection while maintaining a FOV which is larger than just the area of the detector. 


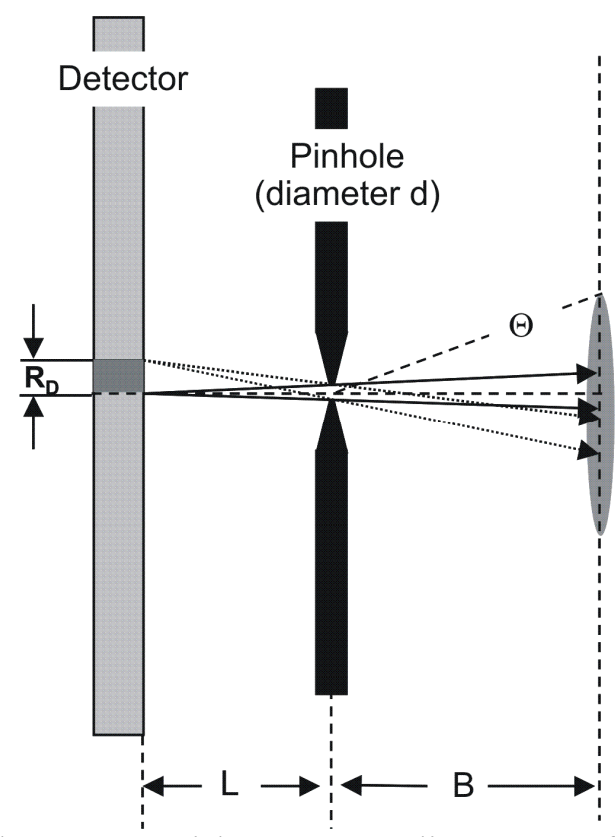

Source

Figure 13: Pinhole system with source at distance B, focal length L, pinhole diameter d, and detector position resolution $\mathrm{R}_{\mathrm{D}}$.

Given the definitions from Figure 13 the geometric resolution $R$ can be expressed as:

$$
R=d+\frac{d}{L} \times B
$$

Taking the intrinsic detector resolution $R_{D}$ into account, the combined resolution $R_{\text {tot }}$ is:

$$
R_{\mathrm{tot}} \approx \sqrt{\left(R^{2}+\left(\frac{B}{L} \times R_{D}\right)^{2}\right)}
$$

The efficiency for a pinhole system for an object at angle $\theta$, which represents the distance from the symmetry axis is:

$$
\varepsilon<\frac{d^{2}}{16 B^{2}} \cos ^{3} \theta
$$

It is important to note that the resolution is proportional to the distance $B$ and the intrinsic resolution $R_{D}$. The degradation in resolution with larger distances can be compensated by a smaller pinhole diameter $d$, however, only with a significant impact on efficiency since the efficiency scales with $d^{2}$. In addition, the efficiency strongly depends on the distance, too. As an example, let's assume an object at $10 \mathrm{~m}$ distance we want to image with $50 \mathrm{~cm}$ resolution (corresponding to $3 \mathrm{deg}$ ). Assuming a focal length of $20 \mathrm{~cm}$ we need a pinhole diameter of $1 \mathrm{~cm}$ to achieve the desired resolution. The efficiency can then be calculated to be $6 \times 10^{-8}$ on the axis. For positions away from the axis $(\theta>0)$ the efficiency is even lower (e.g. at $\theta=20 \mathrm{deg}, 17 \%$ lower). While imaging with a pinhole provides excellent 
imaging capabilities by mapping the object 1:1 to the image plan, it is very limited in terms of efficiency, particularly for larger distances beyond $1 \mathrm{~m}$. Pinhole systems and more recently multi-pinhole systems are used particularly for small animal imaging since the animal can be moved very close to the pinhole and the maximum distance will be in the order of $10 \mathrm{~cm}$ only. Spatial resolution in the order of $1 \mathrm{~mm}$ in small animals has been demonstrated [27].

\subsubsection{Parallel-hole imager}

Parallel-hole imagers such as the Anger camera consist of a heavy absorber, e.g. made of lead or tungsten alloys, with many holes in front of a position-sensitive gamma-ray detector. The holes can have different shapes, ranging from round to hexagonal and they are separated by the septum. Ideally and realized in near-field, each point of the object sees the detector only through one hole, the other pathways suppressed by the collimator and the septa. The collimator resolution is defined similarly to the pinhole:

$$
R=d+\frac{d}{l_{e}} \times b
$$

$d$ is now the hole diameter, $l_{e}$ the effective length of the collimator, and $b$ the distance between front of collimator to object as indicated in . The effective length of the collimator holes takes into account the septal penetration of the gamma rays. The collimator efficiency, e.g. the fraction of gamma rays passing through the collimator per gamma ray emitted into the total opening angle of the collimator, is given by

$$
\varepsilon \approx\left(K \times \frac{d}{l_{e}} \times \frac{d}{(d+t)}\right)^{2}
$$

where $t$ is the septal thickness and $K$ is a constant that depends on the hole shape $(\sim 0.25)$. The resolution improves with long and narrow holes, however, collimator efficiency decreases approximately as the square of the ratio of hole diameter to length, and therefore decreases significantly. An approximate relationship between collimator resolution $R_{C}$ and efficiency $\varepsilon$ is $\varepsilon \propto R_{C}^{2}$. Therefore, for a given septal tickness, collimator resolution is improved only at the expense of decreased collimator efficiency, and vice versa. In biomedical imaging, typically three different sets of collimators are being used, reflecting the different trade offs between resolution and efficiency: High sensitivity, general purpose, and high resolution. Since the attenuation of gamma rays strongly depends on the gamma-ray energy, sets of collimators are available e.g. for low energy such as $140 \mathrm{keV}$ from ${ }^{99 \mathrm{~m}} \mathrm{Tc}$ or medium energy such as $369 \mathrm{keV}$ from ${ }^{131} \mathrm{I}$. Since for higher gamma-ray energies the thickness of collimator and septum has to be increased, the overall sensitivity decreases significantly. Typical values for efficiency and resolution at $10 \mathrm{~cm}$ distance for anger cameras are in the order of $3 \times 10^{-4}$ and $10 \mathrm{~mm}$ for $140 \mathrm{keV}$ and $1 \times 10^{-4}$ and $15 \mathrm{~mm}$ for $369 \mathrm{keV}$. 


\section{The Anger Camera}

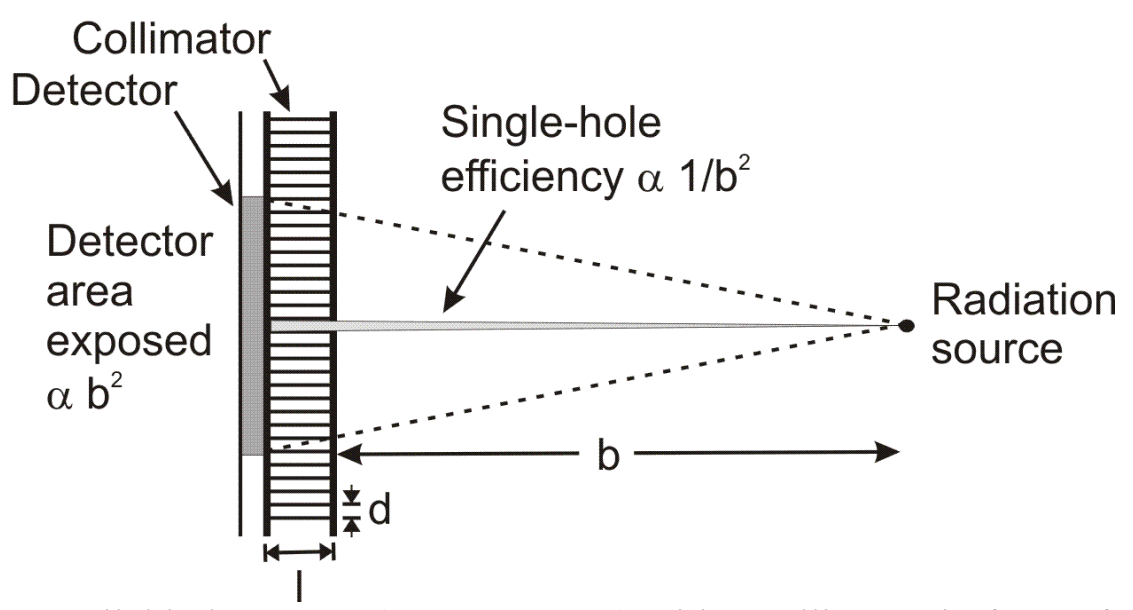

Figure 14: Parallel hole system (Anger camera) with a collimator in front of a position sensitive detector.

As it can be seen from equation 4, the efficiency is independent from the distance of the source. This can be understood by considering and the fact that the loss in single-hole efficiency is compensated by the increased exposed detector area since the number of holes increases through which radiation can pass the collimator. While the efficiency stays constant for larger distances, the resolution deteriorates linearly with the distance. Since Anger cameras are fairly big, they can't be moved very close to specific organs and therefore are limited in sensitivity. New and more compact collimator-based cameras are now on the market which provide higher sensitivity since they can be moved closer to the organ of interest and due improved detector technology which provides higher resolution, e.g. by arrays of small scintillators which are read by position-sensitive photomultipliers or pixilated semi-conductor detectors such as CdZnTe [28]. However, for almost all applications of interest for this report distances are larger than $1 \mathrm{~m}$. As above, if a resolution of $50 \mathrm{~cm}$ at a distance of $10 \mathrm{~m}$ needs to be achieved, and assuming a thickness 1 of $5 \mathrm{~cm}$, then the hole diameter needs to be $2.5 \mathrm{~mm}$. The corresponding efficiency can be determined to be $9 \times 10^{-7}$, which is better than for the pinhole but still very small. Assuming a $1 \mathrm{~m}^{2}$ system with a $1 \mathrm{mCi}$ source in $10 \mathrm{~m}$ distance, we measure one gamma ray every 30 seconds. In contrast to the pinhole system, the parallel hole system has a very limited FOV, in this case about $1 \mathrm{~m}$ at $10 \mathrm{~m}$ distance which is defined by the are of the detector. As noted previously already the multi-hole collimation of a parallel-hole system loses its purpose for larger source distances since the collimator or the septa can not be made long enough to prevent the source to be seen by all the holes as shown in Figure 1.

In addition to these limitations, any collimator-based or aperture-based imaging system has to deal with other image quality degrading factors as well. Figure 15 illustrates these. Gamma ray \#1 is entering the detector through one of the collimators unperturbed and non-scattered. For all gamma rays we observe in the detector we assume this type of event. Gamma ray \#2 hits the detector after penetrating a septum. This is possible since the thickness of collimator and septum can only be finite and transmission of gamma rays 
is possible. Gamma-ray \#3 penetrates the hole of the collimator properly without being scattered before hitting the detector, however undergoes Compton scattering in the detector which can not be resolved. Since most Anger cameras rely on light sharing and center-of-mass determination in one big $\mathrm{NaI}(\mathrm{Tl})$ crystal the deduced position of the gamma ray in the detector will be wrong. Gamma-ray \#4 is scattered in the collimator before being detected in the detector in a wrong location. Finally, gamma ray \#5 is scattered in the object before properly entering the detector.

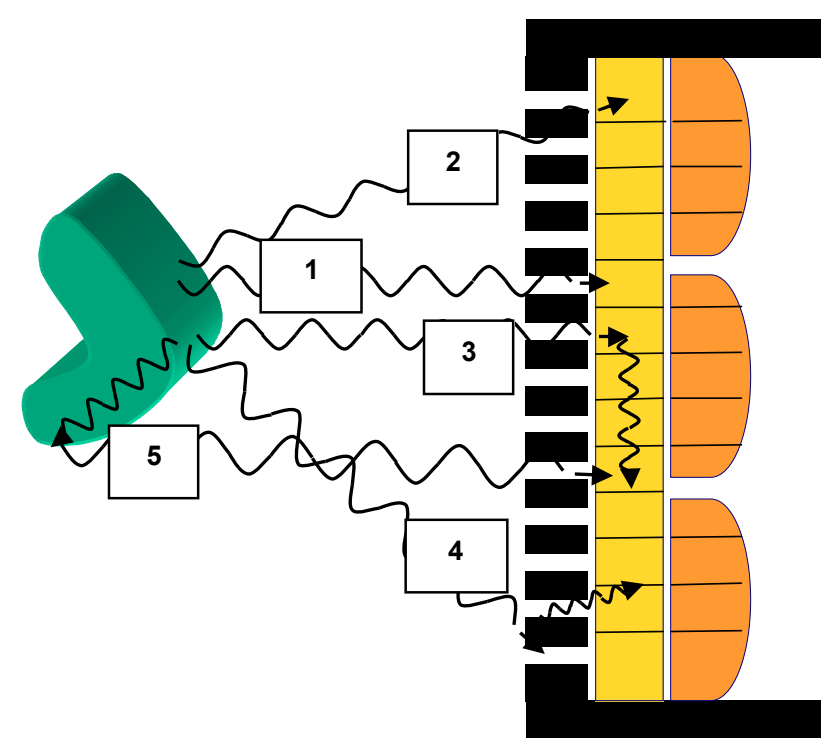

Figure 15: Illustration of a parallel hole collimation system and 5 different types of possible pathways of gamma rays hitting the detector.

Gamma rays events of type 4 and 5 can be effectively reduced by requiring the full gamma ray energy to be detected. In this way, only shallow scattering angles can not be distinguished.

For larger standoff distances, a parallel hole collimator (and for that matter divergent and convergent collimators as well) is not able to sufficiently resolve structure within its FOV. Ultimately, the resolution is equal to the FOV and therefore, no imaging is possible without moving the detector as with proximity imaging. However, then, the parallel-hole collimator is not required anymore and a simple tube-collimator will be more sensitive since no absorber attenuates the gamma-ray flux in the given FOV. A parallel-hole system will therefore be converted to a tube collimator with similar performance as the self-shielding, directional imagers in the previous section. As with active or self-shielding one can envision fairly complex collimator schemes, stationary, rotating, and linearly moving, however, they will be very heavy if high-resolution is required since the collimator needs to be long. For example a FOV or resolution of $10 \mathrm{~m}$ at a distance of $100 \mathrm{~m}$ requires a collimator length of $10 \mathrm{~m}$ for a $1 \mathrm{~m}$ wide detector. We can either reduce the width of the detector and make it more rectangular to maintain the same area or we reduce the length of the collimator which ultimately we lead to a FOV which is the same as without collimation and defined by the attenuation of the gamma rays in air and the 
effective opening angle of the detector. However, the advantage of a collimated and shielded detector is that only gamma rays from the FOV enter the detector and are observed. The penalty is the significantly increased weight to shield and collimate such a system. One could envision an active shield similar to an anti-Compton shield for gamma-ray spectroscopy, now for imaging purposes with a longer collimator at the front.

\subsection{Advanced, modulation systems}

To circumvent some of the problems with classical, collimator based gamma-ray imaging systems such as a pinhole or parallel or tube-like collimators, more advanced modulationbased collimators have been developed. There are two types of modulation imagers: a spatially modulating system, the coded aperture, and the temporary modulating one, the rotation modulation collimator (RMC). We will first discuss the coded aperture approach and then the RMC.

\subsubsection{Coded aperture imager}

\subsubsection{Principle of Operation}

The concept of using a coded aperture was first introduced by Dicke [29] and Ables [30]. In the original formulation the single opening of a simple pinhole camera is replaced by many pinholes (called collectively the aperture) arranged randomly. Each point on a selfluminous object deposits a shadow of the aperture on the picture (see Figure 16).

Subsequent processing of the picture yields the reconstructed image, which should resemble the original object. There are two primary motivations for using a coded aperture approach. The original motivation was to obtain an imaging system, which maintained the high angular resolution of a small single pinhole but produced images that have a SNR commensurate with the total open area of the aperture. The technique is usually applied to x-ray imaging because most $\mathrm{x}$-ray sources are so weak that a single pinhole camera would have to have a very large opening in order to obtain a reasonable SNR. The large hole precludes good angular resolution. If there are $N$ pinholes in the aperture, the picture consists of $N$ overlapping images of the object. The coded aperture technique (for a point source) can improve the SNR by roughly $\sqrt{N}$ when compared to the single pinhole camera. Since $N$ might be as large as 105 , the goal of an improved SNR is obtainable. The second primary motivation is to perform tomography as shown by Barrett [31]. Object points at different distances from the aperture will cast shadows of the aperture onto the picture with different over-all sizes. One can reconstruct a particular depth in the object by treating the picture as if it was formed by an aperture scaled to the size of the shadow produced by the depth under consideration. This property of coded apertures is particularly beneficial in medical applications, although uses in industrial inspection can also be easily envisioned. 


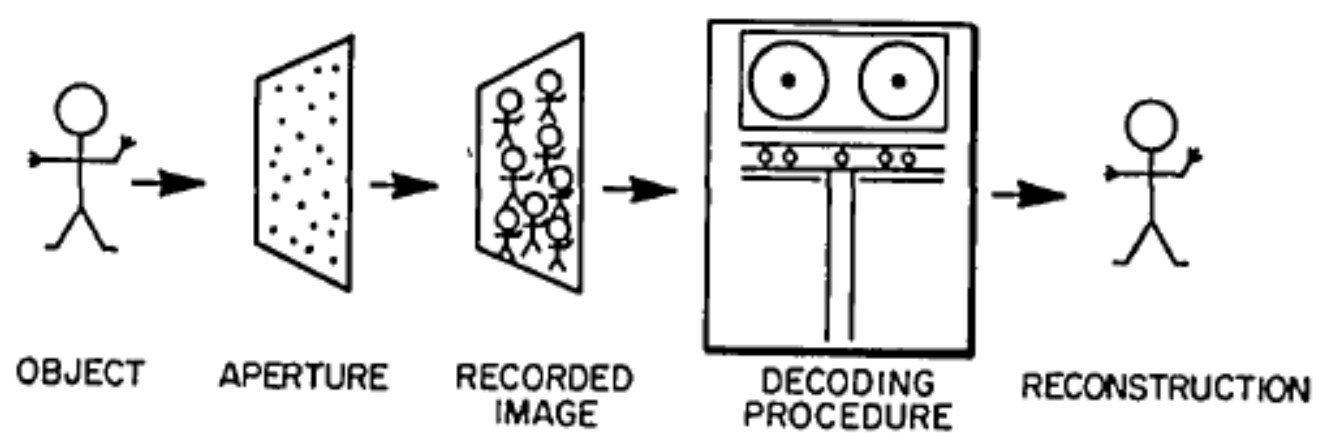

Figure 16: The basic steps involved in coded aperture imaging are shown above. In an attempt to obtain a higher SNR, a multiple-pinhole aperture is used to form many overlapping images of the object. The resulting recorded picture must be decoded, using either a digital or optical method. The resulting reconstruction is of higher quality than that obtained by using a simple pinhole.

The recorded picture is not recognizable as the object because the many pinholes cause the picture to consist of many overlapping images. In order to be useful, the picture must be subjected to a reconstruction method, which will compensate for the effects of the imaging system. The reconstruction procedure is designed to give the location and intensity of each source in the field of view. Basically this is accomplished by detecting the location and strength of aperture patterns in the picture. The analysis methods developed so far can be categorized as either a deconvolution or a correlation. If the recorded picture is represented by the function $P$, the aperture by $\mathrm{A}$ and the object by 0 ,

$P=(O * A)+N$,

where $*$ is the correlation operator and $N$ is some noise function. In the correlation method the reconstructed object is defined to be $[32,33]$ :

$Q=P * G=R O^{*}\left(A^{*} G\right)+N^{*} G$

where $\mathrm{G}$ is called the postprocessing array and is chosen such that $\mathrm{A} * \mathrm{G}$ approximates a delta function. In general, we do not mean $\mathrm{G}$ to be the convolutional inverse function (A'), rather $\mathrm{G}$ will be selected in an ad hoc manner such that $\mathrm{A} * \mathrm{G}$ has desirable properties. Normally $\mathrm{G}$ will be a binary array (as is A). If $\mathrm{A} * G$ is a delta function, $=0+N^{*} \mathrm{G}$, and the object has been perfectly reconstructed except for the presence of the noise term.

Note that the noise term above will not have singularities as in the deconvolution method. The original expectation of obtaining a roughly sqrt(N) improvement in the SNR has not been realized because $\mathrm{A} * G$ in general will not be a delta function. A point on the object will contribute to the reconstructed object the distribution $A * G$ instead of a delta function. Thus, even if there is no background noise and the source is intense enough such that shot noise is not a problem, the SNR for a point source becomes a fixed number regardless of the exposure time. The SNR becomes the ratio of the central peak in $\mathrm{A} * \mathrm{G}$ to the noise in $\mathrm{A} * G$, that is, the square root of the variance of the sidelobes. This noise is 
called the inherent noise [32] and puts a limit on the possible SNR improvement. The situation is much worse when the object is not a point source but is extended. In the extended case, the inherent noises from all points in the object contribute noise to each point in the reconstructed object. The result is a low SNR which cannot be improved because the noise is set by the structure in $A * G$ rather than counting statistics or background levels.

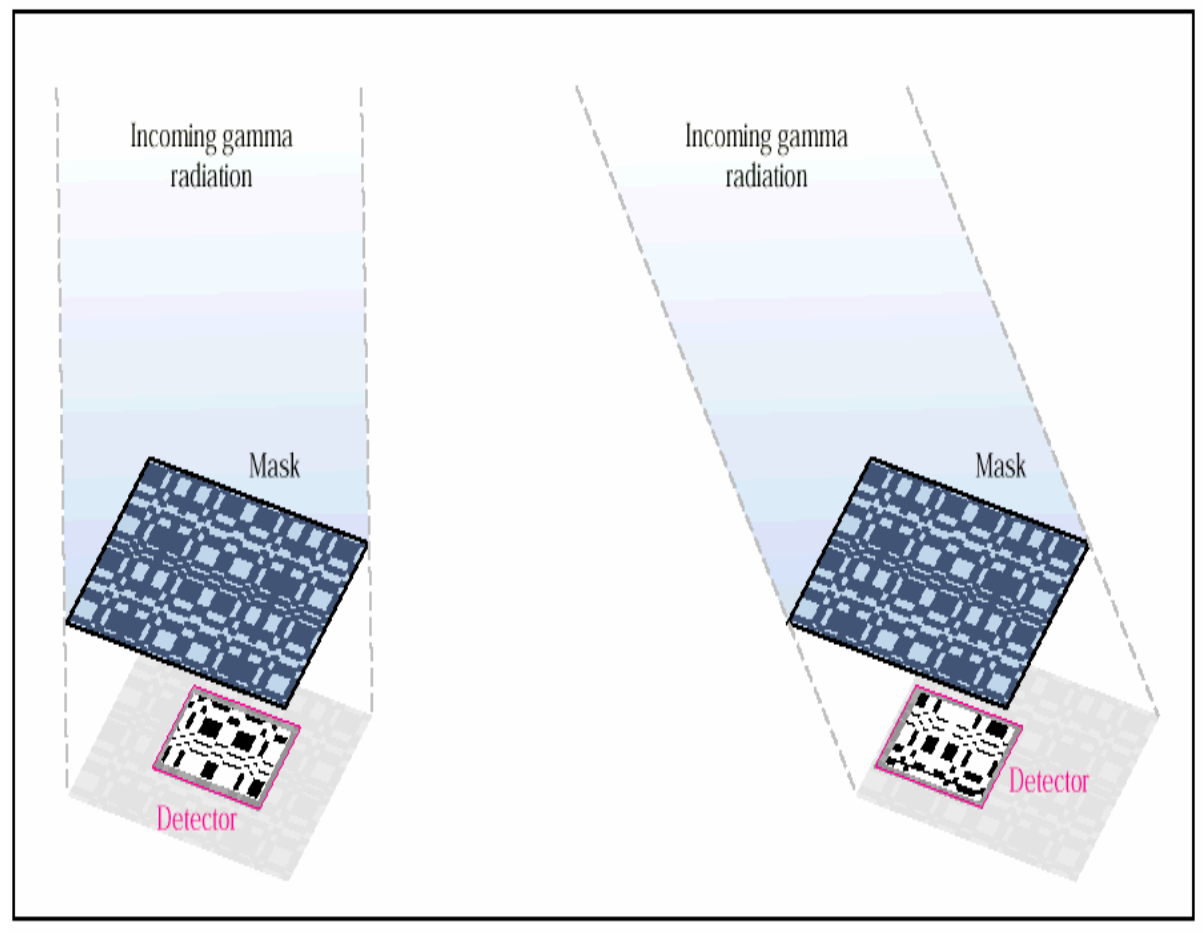

Figure 17: Coded aperture demonstration. 


\subsubsection{Coded Aperture Systems}

\subsection{LLNL GRIS}

A photograph of the LLNL Gamma-Ray Imaging System GRIS is shown in Figure 19 [34]. It comprises a positionsensitive detector mounted on a linear bearing; a hex, uniformly redundant-array, coded aperture mask; shielding and the data acquisition system. The instrument can be set to any of five preset focal lengths providing a factor of $3.7 \mathrm{zoom}$. The mask is cut from $0.5-\mathrm{mm}$ thick tantalum sheets, 16 of which are laminated to form the total $0.8 \mathrm{~cm}$ thickness. It is based on a rank-9 HURA (hex uniformly redundant array) [35] with a single pixel hex sidelength of $2.43 \mathrm{~mm}$ and a center-to-center spacing of 4.21

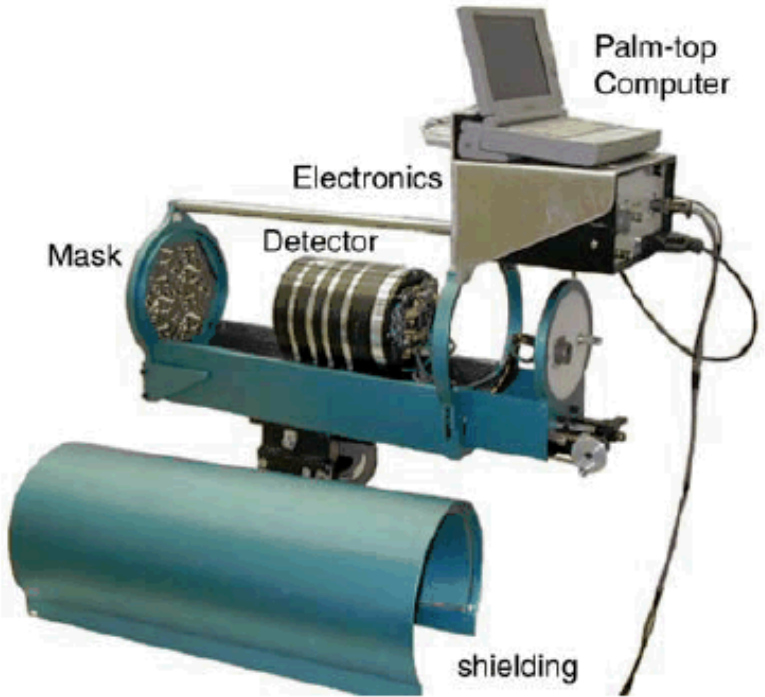

Figure 19: The LLNL Gamma-Ray Imaging System GRIS.

$\mathrm{mm}$. Lead shielding $9.5 \mathrm{~mm}$ thick is permanently mounted below the linear slide. Top and side shielding is provided by a separate partial-cylinder, $9.5 \mathrm{~mm}$ thick which can be slid over the imager. The detector is based on a Hamamatsu R3292, 10-cm-diameter active-area position-sensitive photomultiplier tube (PSPMT) [36] coupled to a 1-cm-thick $\mathrm{CsI}(\mathrm{Na})$ crystal. The acquisition system uses custom gated integrator circuits coupled into an eight-channel 16-bit digitizer card. The instrument is controlled by a modified version of commercial software obtained from RMD Corporation [37]. In developing this instrument, the goal was to maintain the 1917 resolution elements (37 33 pixels) of the current coded aperture imager. To obtain the requisite 36 pixels across the $10-\mathrm{cm}-$ diameter PSPMT requires a resolution of order $3 \mathrm{~mm}$. In practice, the available detector diameter is less than $10 \mathrm{~cm}$ because the performance of the system degrades at the edges. In addition, the mask pattern at the detector is magnified when acquiring images of objects at finite distances. (The degree of magnification is a function of the resolution at the target and must be accounted for before the image deconvolution is undertaken.) The detector uses a Hamamatsu R3292 PSPMT photomultiplier tube coupled to a 1-cmthick 12-cm-diameter CsI $(\mathrm{Na})$ crystal. The crystal (specular polish on all faces) is glued to the PSPMT face using Rexon RX-22P coupling compound [38]. Graphite-blackened silicone compound is used to pot the edges of the crystal and reduce light cloud reflection and position distortion as outlined below. The top face is covered with Teflon micropore tape and a 125- m-thick aluminum window is used to hermetically seal the system. 
Further details on the selection and preparation of the crystal can be found in reference [39].

To improve spectroscopic and image resolution, a system consisting of a coded aperture, a double-sided strip detector (DSSD) made of HPGe and a coxial HPGe detector was built. Figure 20 shows a photograph of this system. The coaxial detector behind the planar DSSD detector is used for higher gamma-ray energies to catch the gamma ray which was scattered in the DSSD system. In this way, the position of the incident gamma ray can uniquely be measured in the DSSD system. The drawback of this approach is the significantly decreased efficiency, ultimately similar of that of a Compton camera.

However, for low gamma-ray energies $(<150$ $\mathrm{keV}$ ) where predominantly photo-electrics absorption occurs, the DSSD system will provide all the information needed. Extensive measurements have been performed over the last two years with this system and improved image as well as energy resolution was demonstrated [40].

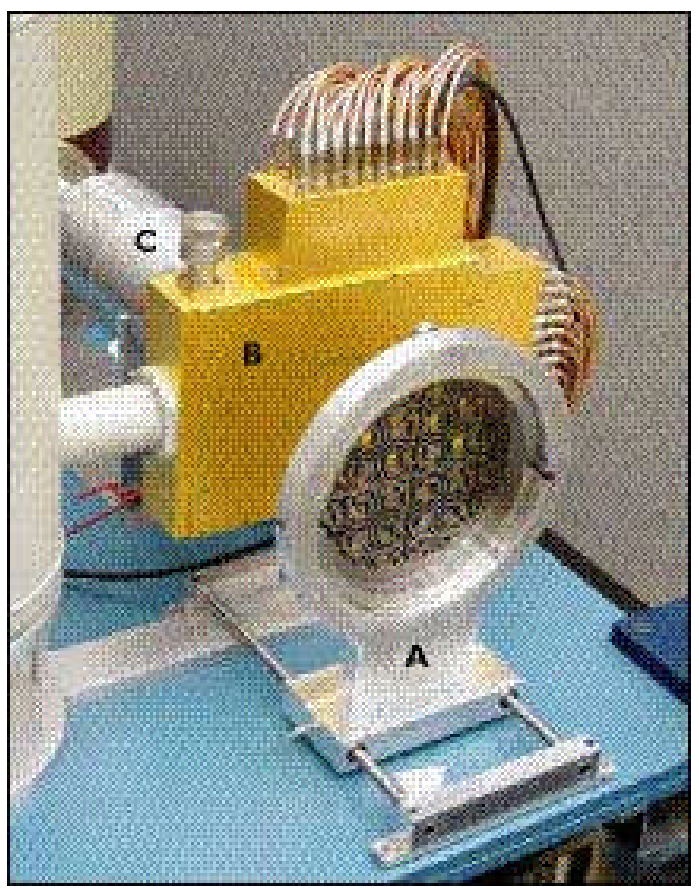

Figure 20: New LLNL coded aperture imager consisting of coded aperture, one DSSD and one coaxial HPGe detectors.

\subsection{LLNL Large Area Imager}

\subsection{First Prototype}

A large-area, coded aperture gamma-ray imaging system was developed as shown in Figure 21 to demonstrate the ability to image gamma-ray sources at distances at about $100 \mathrm{~m}$. This imaging system is designed around an extant set of $57,10 \mathrm{~cm} \times 10 \mathrm{~cm} \times 10$ $\mathrm{cm} \mathrm{NaI(Tl)} \mathrm{detectors.} \mathrm{These} \mathrm{are} \mathrm{arranged} \mathrm{in} \mathrm{a} 3$ x 19 array and are located behind a onedimensional, base-19, uniformly-redundant array, coded-aperture mask [41]. The details of the design choices are provided in [42]. Briefly, the number of pixels in the imager is determined primarily by the length of time that an object is in the instrument's field of view, given its one meter focal length. This size is selected as a compromise between vehicle width and the imager resolution at $100 \mathrm{~m}(10 \mathrm{~m})$. The imager provides only onedimensional images - a design choice based on its intended use in suburban or lighturban areas. Searches in areas with high-rise buildings beyond three stories are significantly complicated by the extra shielding from the concrete floors prevalent in their designs. The mask comprises individual elements of linotype metal $(85 \% \mathrm{~Pb}, 11 \% \mathrm{Sb}, 3$ 
$\% \mathrm{Sn}$, and $1 \%$ other materials) that are $4 \mathrm{~cm}$ thick by $10.8 \mathrm{~cm}$ wide by $100 \mathrm{~cm}$ high. This material was selected for its lead-like stopping power with improved mechanical properties. The individual elements are supported in a steel frame that allows for rotation of sections of the mask around axis of antisymmetry. Additional mask elements may be lowered into or out of the field of view of the detector (Figure 21). These features enable to exchange the mask for its inverse (anti-mask) in sequential passes past a source, providing an important means of noise control as explained later. The detectors are supported from a plate mounted in a steel frame similar to that used for the mask. The detectors are separated from the plate with a $0.5 \mathrm{~cm}$ thick sheet of elastomer to help protect them from mechanical and thermal shock. An additional $2.5 \mathrm{~cm}$ of Styrofoam is placed on the front, tops, sides and bottom of the detector array as thermal insulation. Several pieces of aluminum channel connect the mask and detector frames to fix the one meter focal spacing and to restrict motion of one with respect to the other. The instrument relies on friction to keep it from moving within the truck. A safety strap ties the mask to the rear of the vehicle to protect the cab occupants in the event of a sudden stop. The instrument weighs $1585 \mathrm{~kg}$ with the $1225 \mathrm{~kg}$ mask comprising the bulk of this mass. The overall length of the mask is four meters.

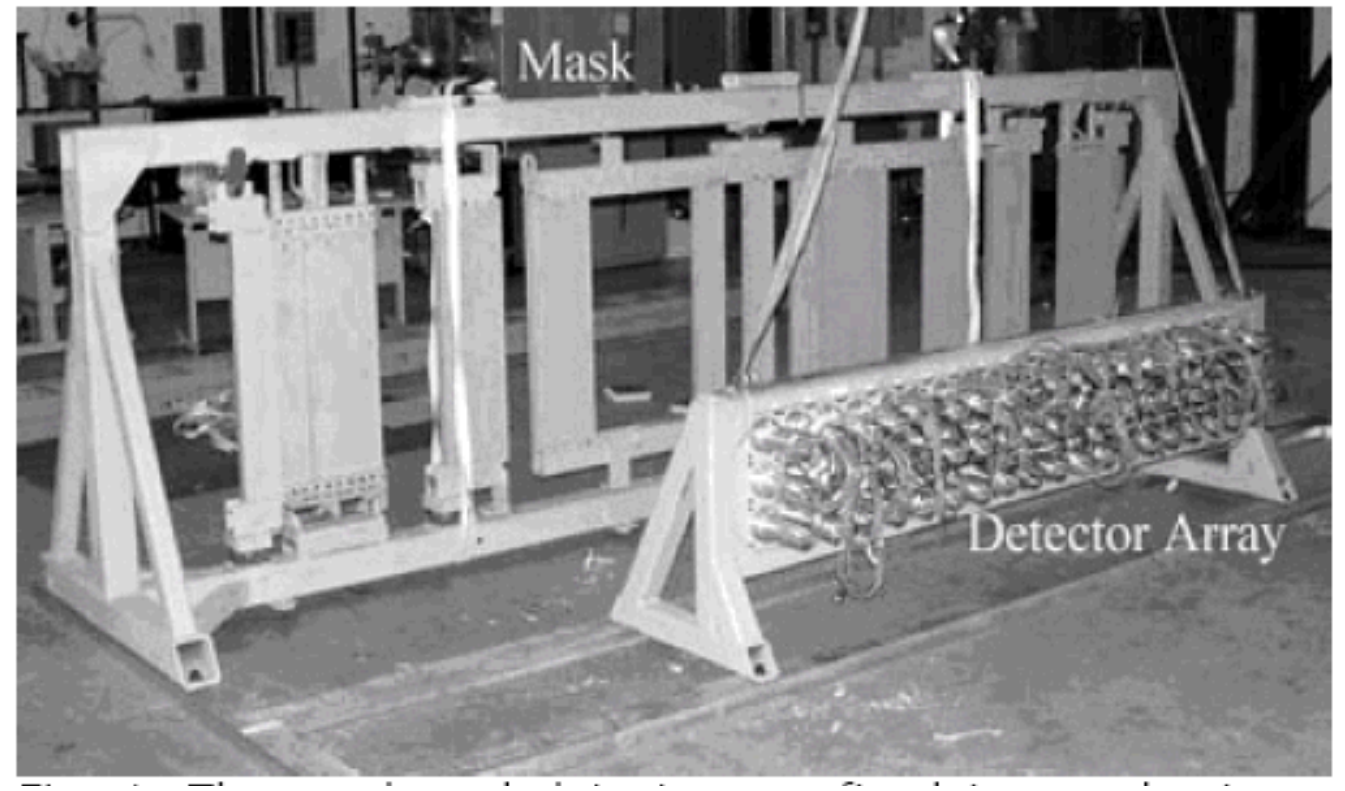

Figure 21: The mask and detector are fixed to an aluminum frame that maintains the imager focal length and alignment. The system is mounted to look through the curb side of a vehicle. 


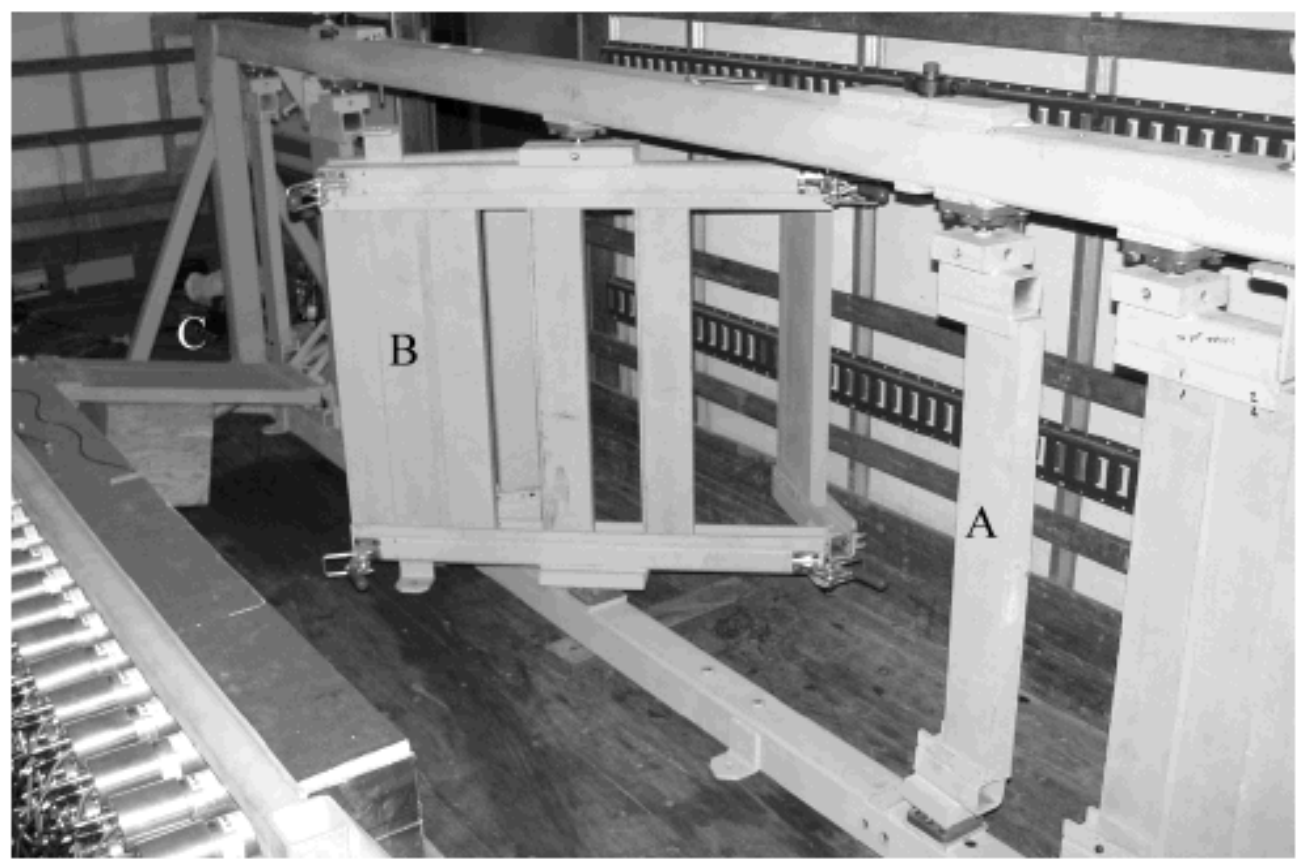

Figure 22: The mask is converted to the anti-mask by rotating different parts about axis of anti-symmetry ( $\mathrm{A}$ and $\mathrm{B}-\mathrm{B}$ is hinged to reduce the swing space required) or by lowering a section out of the field of view (C).

\subsection{Extended Prototype}

An extended configuration of the first prototype includes two detector arrays. One is placed behind a mask collimator array, the other behind an antimask. The combination of the reconstructed mask/antimask images increases the sensitivity of detection, especially for extended sources.

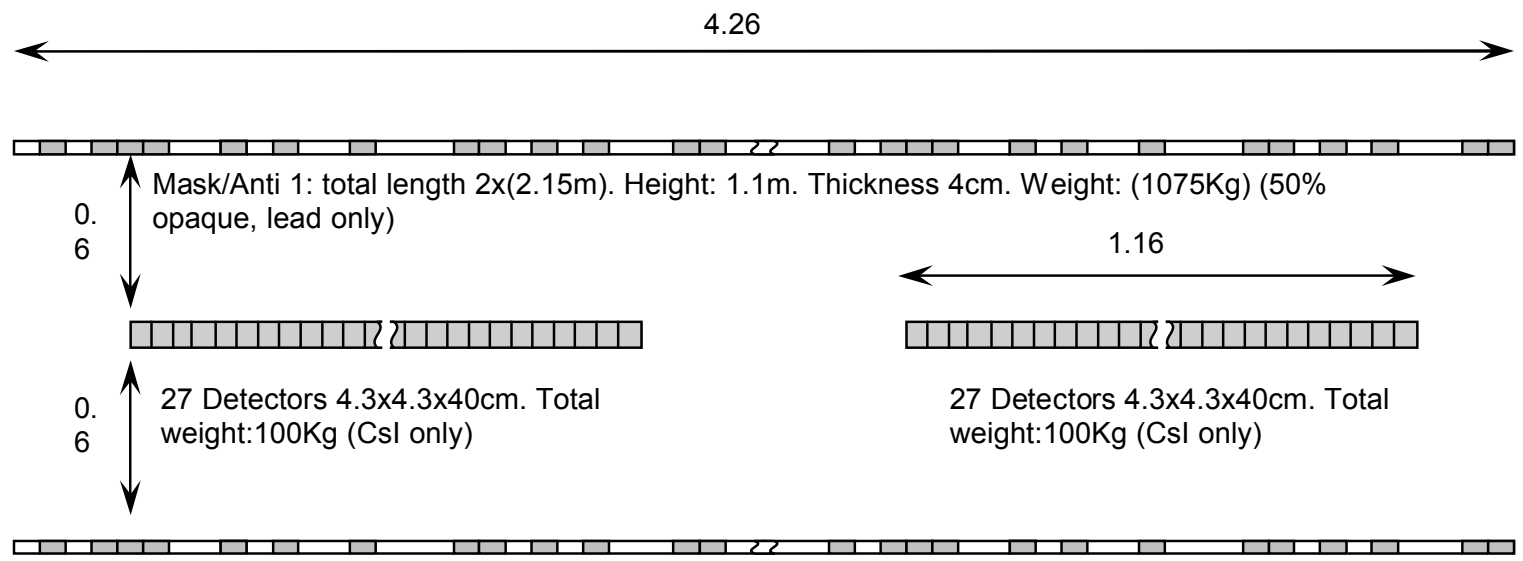

Mask/Anti 2: total length $2 x(2.15 \mathrm{~m})$. Height: $1.1 \mathrm{~m}$. Thickness $4 \mathrm{~cm}$. Weight: $(1075 \mathrm{Kg})(50 \%$ opaque, lead only)

Figure 23: Extended prototype contains a mask and antimask arrays. 
Basic data on the extended configuration:

- Uses $54 \mathrm{CsI}(\mathrm{Na})$ bars $4.3 \mathrm{~cm}$ x $4.3 \mathrm{~cm}$ x $40 \mathrm{~cm}$

- Detector area is $0.93 \mathrm{~m}^{2}$

- Based on a 23 element coded aperture

- 23 pixels in the image

- 27 pixels in the mask 50 in the detector to handle magnification issues

- Mask pixel size is $4.27 \mathrm{~cm}$

- This provides a unitary mask sampling at the farthest range of $95 \mathrm{~m}$

- Focal length $62 \mathrm{~cm}$

- $6.5 \mathrm{~m}$ pixels at $95 \mathrm{~m}$ from system

- $150 \mathrm{~m}$ field of view at $95 \mathrm{~m}$

- Dual system using linear mask and anti-mask imagers

- Dual masks will image both sides of the road simultaneously

- Source appears positive on the correct side negative on the other

- Overall Length $\sim 4.3 \mathrm{~m}$ and width $\sim 1.4 \mathrm{~m}$

- Mass

- $2150 \mathrm{~kg}$ lead for mask (1.1m high)

- $200 \mathrm{~kg}$ for crystals

- $200 \mathrm{~kg}$ hardware

\subsection{INTEGRAL SPI}

The high-resolution $\gamma$-ray spectrometer (SPI) is one of the two main instruments on board the ESA INTEGRAL satellite successfully launched in October 2002 [43]. SPI uses coded aperture mask technique in order to have imaging capabilities at the energy band (20 keV-8 MeV) it will study celestial sources. The flight model of SPI was delivered to ESA in May 2001. The core of the instrument is the gamma camera composed of a hexagonal array of 19 high purity germanium detectors with geometrical area of $508 \mathrm{~cm}^{2}$. As these detectors have to work at low temperature (in the range 85-90 K), an active cryogenic system is used to continuously cool them. Under these conditions the energy resolution for gamma-ray spectroscopy is close to the best which can be achieved today in laboratory, at least 20 times better than the energy resolution of the detectors used in the last two major - scintillator-based - gamma ray astronomy missions. Even though an active cooling system is technically difficult to operate in space, its redundancy would allow to expect a mission duration of several years. For imaging, a coded mask made of tungsten blocks is associated with the Ge array. A compromise between the weight and the opacity of the tungsten blocks in the $\mathrm{MeV}$ range led to a thickness of $3 \mathrm{~cm}$ for the blocking elements of the mask. To increase the sensitivity of the telescope, the background due to cosmic ray interactions with the Ge detectors and all the materials around them has to be reduced. This is achieved with an active anticoincidence system (ACS) made from BGO crystals. The thickness of the BGO crystals was optimized after Monte Carlo simulations of the background [44, 45] because too great a mass of BGO around the detectors can increase secondary neutron production, producing extra background in the Ge crystals. The ACS also defines the field of view of the telescope. As SPI is designed to be able to map extended structures, for instance the ${ }^{26} \mathrm{Al}$ or $511 \mathrm{keV}$ 
galactic distributions, the field of view has to be large. The chosen fully coded field of view is 16 degrees, with a distance between mask and camera of $1.71 \mathrm{~m}$. This provides an angular resolution of about 2.5 degrees, although sources can be localized much better, to about 10 arcmin depending on the source intensity. Complementary data from IBIS can be used to localize the sources seen by SPI below $200 \mathrm{keV}$ even more precisely $(\sim 1$ arcmin), if the source emits in this energy range. For background reduction at $511 \mathrm{keV}$, simulations showed the interest of adding a thin plastic scintillator just below the tungsten mask. The Plastic Scintillator Anticoincidence subassembly (PSAC) allows the rejection of background induced by prompt interactions in the mask due to particles which do not reach the BGO ACS because of a gap of $70 \mathrm{~cm}$ between mask and ACS (this gap is enforced by weight constraints). An important source of background which has been identified during the study of gamma-ray detector backgrounds $([46,47])$ are $(n$, $\beta$ ) interactions which dominate the background between $200 \mathrm{keV}$ and $2 \mathrm{MeV}$. If $\beta$ interactions are not accompanied by a detected gamma-ray in the Ge detector they produce a localized energy deposit. They can then be distinguished from interactions of gamma rays which, in this energy range, interact predominantly by Compton scattering followed by a photoelectric interaction of the scattered photon. Such interactions deposit

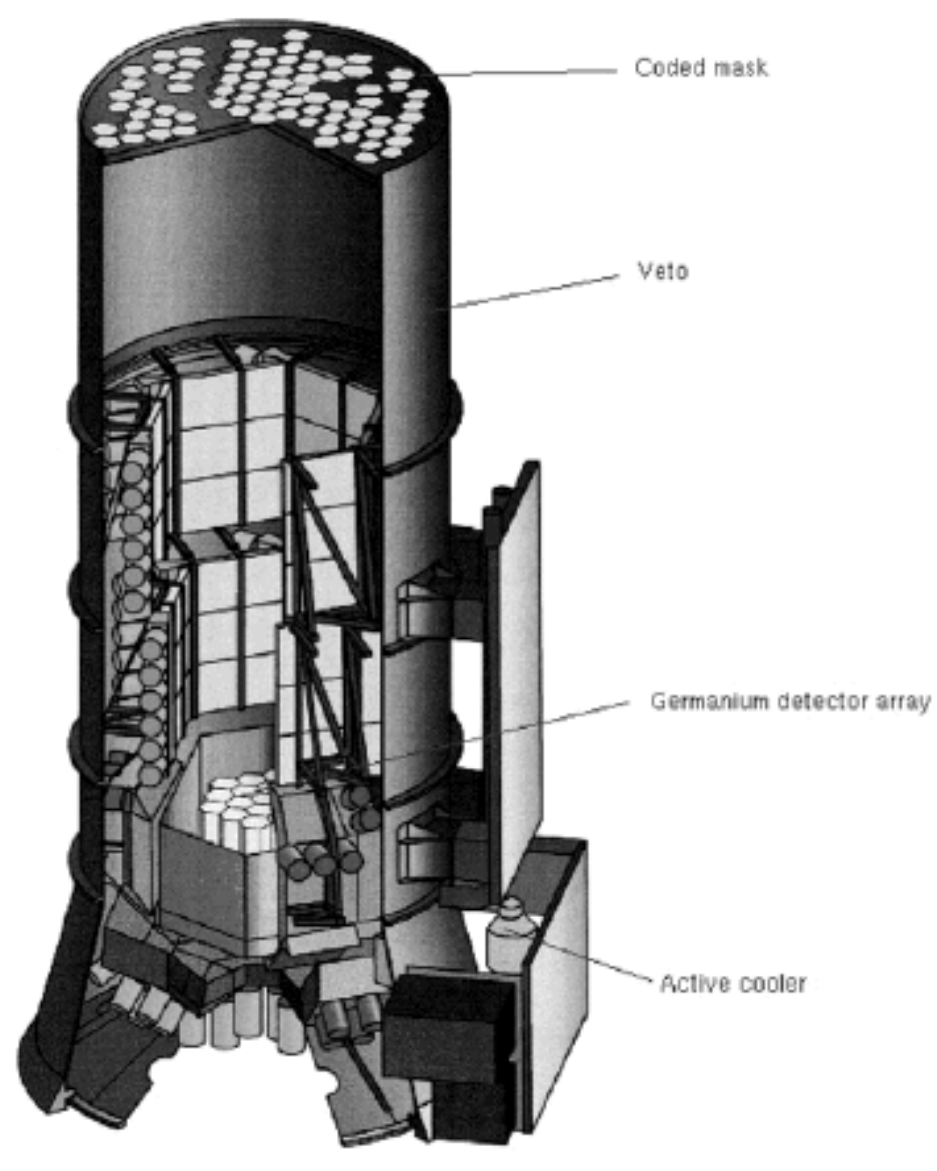
energy in two or more sites. So $\beta$ decays are mostly single site events and most gamma-ray interactions are multiple site events. The shape of the pulses delivered by these two types of events is different and they can be recognized by a pulse shape discrimination (PSD) system. Such a device has been implemented on SPI - this is the first time that this new way to reduce the background between 200 $\mathrm{keV}$ and $2 \mathrm{MeV}$ is tried for Ge detectors onboard a satellite. The operating mode of SPI is based on the detection of Ge detector events which are in the energy range $20 \mathrm{keV}-8$ $\mathrm{MeV}$ and which are not accompanied by ACS events.

Figure 24: The spectrometer SPI 
The SPI mask, which is situated at $1.71 \mathrm{~m}$ above the detection plane is a circular passive array of 127 hexagonal pixels of which 63 are opaque and 64 transparent to gamma radiation within the operational energy range (Figure 24). The HURA (Hexagonal Uniform Redundant Array ) coded mask pattern has 120 deg symmetry and is inscribed in a $720 \mathrm{~mm}$ diameter circle. The cells are $60 \mathrm{~mm}$ side to side, the same as the detector module pitch. The opaque cells are made of a tungsten alloy (density $18 \mathrm{~g} \mathrm{~cm}^{-3}$ ) and are $30 \mathrm{~mm}$ thick. This allows an opacity better than $90 \%$ over the entire energy range. The tungsten cells are mounted on a carbon fiber sandwich assembly. The measured positioning accuracy (mean deviation over the theoretical position) of such tungsten cells was $0.04 \mathrm{~mm}$ (the required value during the design phase was $0.15 \mathrm{~mm}$ ).

To align the detection axis of SPI it was decided to have a central hole in the mask to enable a fiducial mark on the cryostat to be sighted through the mask and the PSAC which is just below the mask. This hole (diameter $10 \mathrm{~mm}$ ) is reinforced with potting (external diameter $28 \mathrm{~mm}$ ) and titanium washers (diameter, $40 \mathrm{~mm}$ ) on both lower and upper parts of the sandwich panel. This structure decreases the transparency of the central element of the mask with respect to the rest of the open mask elements (from $82 \%$ to $59 \%$ at $60 \mathrm{keV}$ and from $88 \%$ to $79 \%$ at $511 \mathrm{keV}$ ). Except for this central pixel the transparency of the mask is in agreement with the specifications. For instance at 0degrees the transparency is $75 \%$ at $35 \mathrm{keV}$, reaching $85 \%$ at $300 \mathrm{keV}$ [48]. The mask weight is about $140 \mathrm{~kg}$.

Performance and engineering characteristics of SPI:

$\begin{array}{ll}\text { Energy range } & 20 \mathrm{keV}-8 \mathrm{MeV} \\ \text { Energy resolution } & 2.5 \mathrm{keV} \text { at } 1.33 \mathrm{MeV} \\ \text { Angular resolution } & 2.5 \text { degrees } \\ \text { Positioning accuracy } & 10 \text { arc min } \\ \text { Fully Coded FOV } & 16 \text { degrees } \\ \text { Detector area } & 500 \mathrm{~cm}^{2} \\ \text { Detector thickness } & 7 \mathrm{~cm} \\ \text { Total weight } & 1228 \mathrm{~kg}\end{array}$

\subsection{Small Animal Imager}

The design of detectors for radio-imaging of small animals is challenging because of the high spatial resolution required, possibly coupled with high efficiency to allow dynamic studies. Spatial resolution and sensitivity are difficult to attain at the same time with single photon imaging techniques because collimators define and limit performance. In this paper we describe a simple desktop gamma imager equipped with a pinhole collimator and based on a pixellated $\mathrm{NaI}(\mathrm{Tl})$ scintillator array coupled to a positionsensitive photo-multiplier (PSPMT). The limits of such a system as well as the way to overcome them in future systems are shown next. Better light sampling at the anode level would allow better pixel identification for a higher number of pixels, which is one of the parameters defining image quality and improving spatial resolution. Finally, we show how the substitution of the pinhole collimator with a coded aperture collimator can result 
in a substantial improvement in system sensitivity while maintaining very good spatial resolution, possibly at a sub-millimeter level. Calculations and simulations of a particular solution show that sensitivity can improve by a factor of nearly 30 . A variety of pixilated $\mathrm{NaI}(\mathrm{Tl})$ detectors with different pixel sizes and pixel sizes were evaluated as well the readout with a variety of different PSPMTs. The position of each scintillation event was calculated with a digital centroid technique applied to the individual anode amplitudes. Parallel hole as well as pinhole and coded aperture collimators were used. The parallel hole collimator was a standard general purpose lead collimator (hole diameter $1.5 \mathrm{~mm}$, hole length $22 \mathrm{~mm}$, and septa thickness $0.2 \mathrm{~mm}$ ). The two tungsten pinhole collimators had a knife aperture with an angle of 60 degrees and hole diameters 1 and $0.67 \mathrm{~mm}$. Employing the coded aperture system we achieved a sensitivity that is 30 times higher than that obtained with a single pinhole collimator. The theory of coded aperture signalto-noise ratio (SNR) [49], [50] shows that this advantage is fully translated to an increase in the SNR only in the case of a point source. In the opposite limiting case of a uniform flood field, the SNR provided by a pinhole collimator is higher. The case of a hot spot with background activity, typical of many small animal imaging measurements, is intermediate to the two above extreme cases, but theoretical calculations, computer simulations, and previous experimental experience show that the increased sensitivity of coded apertures can actually translate to increased SNR, without sacrificing resolution [50].
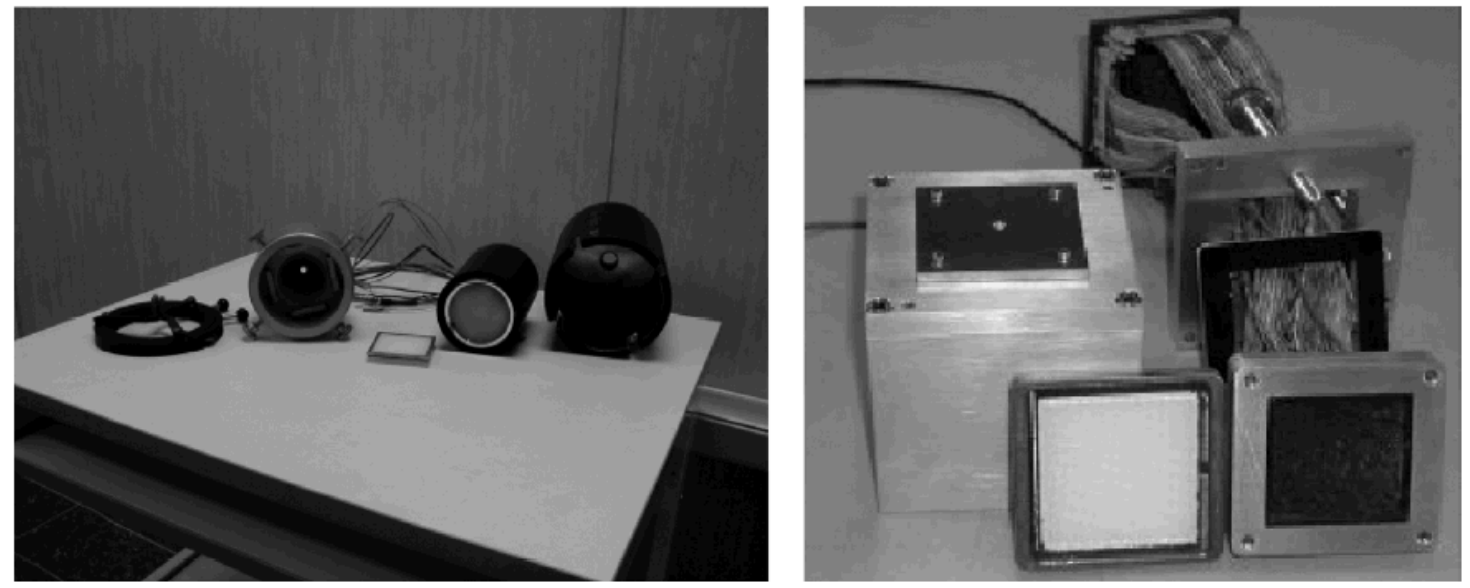

Figure 25: Two detector setups using different photodetectors. At left: $\mathrm{NaI}(\mathrm{Tl})$ pixellated array $(1.8 \times 1.8 \times 6 \mathrm{~mm})$ coupled with R2486 Hamamatsu PSPMT using pinhole collimator. At right: The same system but using H8500 PSPMT as photodetector. 


\subsubsection{Rotation modulation imager}

\subsubsection{Introduction}

The concept of the Rotation Modulation Collimator (RMC) was first proposed in the last 1960's as an improvement over scanning collimation for hard x-rays and soft $\gamma$-rays [51]. Soon afterwards, Schnopper et. al. suggested its use for x-ray astronomy. Thereafter, it has been successfully employed in both rocket flights $[52,53]$ and balloon-based experiments $[54,55]$ for hard $\mathrm{x}$-ray imaging.

The RMC was used in the late 1970's on a balloon-based experiment as a wide field of view $\gamma$-ray burst detector [56]. This decade also saw three satellite missions employing RMCs; Ariel 5, SAS-3, and Hakuch were all launched.

Interest grew in the 1980s for using RMCs to study solar physics [57]. At present, the most sophisticated RMC yet built is on-board the RHESSI satellite [58]. RHESSI is using an RMC to study solar flares in the x-ray and $\gamma$-ray regime.

To date, all applications of the RMC have been on balloon or satellite experiments. To the best of our knowledge, no RMC's have been used for terrestrial applications.

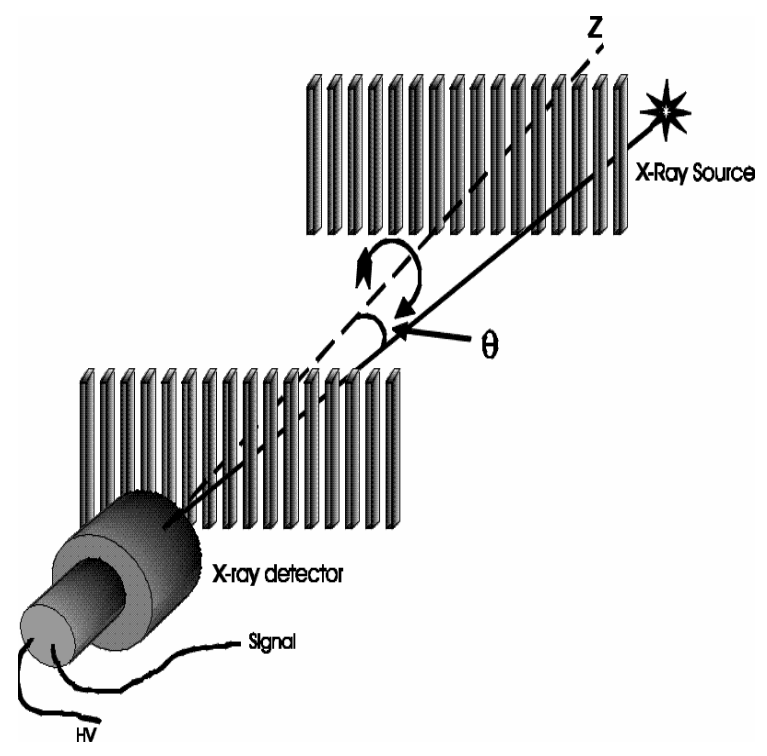

Figure 26: Basic concept of the Rotation Modulation Collimator. The collimator consists of two sets of parallel bars which rotate together in front of the detector. As the collimator rotates, it modulates in time the signal arriving from the source. This modulation is a function of source position and can be reconstructed with the appropriate algorithm.

\subsubsection{Brief System Description}

The RMC utilizes a detector behind two widely spaced grids. Each grid is a series of alternating slits and slats. That is, each grid is a series of parallel bars that either block the incoming radiation or allow it to pass. The bars must be made of a high- $\mathrm{Z}$ material such that they sufficiently attenuate the energies of interest. The transmission through the grids 
is thus partial $(\sim 50 \%)$ and periodic in the direction orthogonal to the slits. The collimation grids are constantly rotated through $180^{\circ}$ which results in the modulation of the source count rate. Figure 26 illustrates the basic arrangement. The speed of rotation is not important as long as the source is not changing (in position or intensity) on the time scale of the rotation. The resolution of an RMC is defined as $1 / 2(p / L)$ where $p$ is the slit/slat pitch and L is the length, or separation, between the two collimator planes. Depending on the design and energies of interest, the resolution can range from $\sim 1^{\circ}$ to a few arcseconds.

One of the advantages of the RMC technique, over other imaging modalities, is its relative simplicity. In particular, only a single detector is required. Furthermore, the detector needs not be position sensitive. This is in contrast to a coded aperture system which requires a position sensitive detector. This requires many electronic channels with their associated cost and complexity. Furthermore, the position resolution of a coded aperture is fundamentally limited by the pixel size. Thus, very fine resolution (arcminutes or less) is not achievable because of the small pixel size required. For an RMC system, on the other hand, the only requirement is that the detector has both high stopping power and good energy resolution. For $\gamma$-rays, the stopping power can be readily achieved by standard scintillation detectors such as $\mathrm{CsI}$ or NaI or other more advanced materials. However, the resolution of a germanium detector greatly aids in isotope identification and background rejection. A CZT based system might also be viable, offering good stopping power, reasonable resolution and cryogen-free operation. Silicon and gas-based detectors, however, do not offer sufficient stopping power and are not viable for an RMC.

\subsubsection{Image Reconstruction}

The rotation of the collimator results in the modulation in time of the source signal. Specifically, the RMC measures the Fourier components of the source distribution. The frequency of modulation is proportional to the radial offset of the source; the overall phase depends on the azimuthal position; the modulation amplitude depends on the total flux of the source [59]. For multiple sources, the modulation pattern is a linear superposition of individual patterns. Reconstructing the image then requires solving the inverse problem. Fortunately, this is mathematically equivalent to the problem in radio interferometry and the algorithms used in this field can be applied to RMC image reconstruction.

The basic algorithm is simple back projection. Back projection involves solving the $2 \mathrm{D}$ inverse Fourier transform [60]. The resulting image estimates the convolution of the source with the instrumental response. Back projection is linear in that projections from different time intervals may be added together resulting in an improved image. The disadvantage of back projection is that it has artifacts ('side lobes') which do not improve with increased data.

Improvements to the image can be achieved by employing iterative algorithms. The two most common are the Clean algorithm and Maximum Entropy. Clean was originally developed for radio astronomy and is based on the assumption that the image can be well 
represented by a superposition of point sources [58]. However, in many cases it can be used for extended sources as well. Clean is an iterative algorithm that begins with the back projection as the first estimate. The algorithm identifies the largest pixel in the map with the largest amplitude: $F_{j}$. This pixel is then normalized by the point spread function and subtracted from the image. The process is then repeated on the residual image with the next largest point source being removed (again, assuming a map of point sources). This continues until the resulting image is determined to contain only noise.

The Maximum Entropy Method (MEM) seeks to find a map that is consistent with the data and maximizes the "entropy" of the measurement. MEM results in an image that is 'smooth' compared to other techniques. The main advantage of MEM is its efficiency and lower memory requirements. A detailed mathematical description of both Clean and MEM can be found in [58].
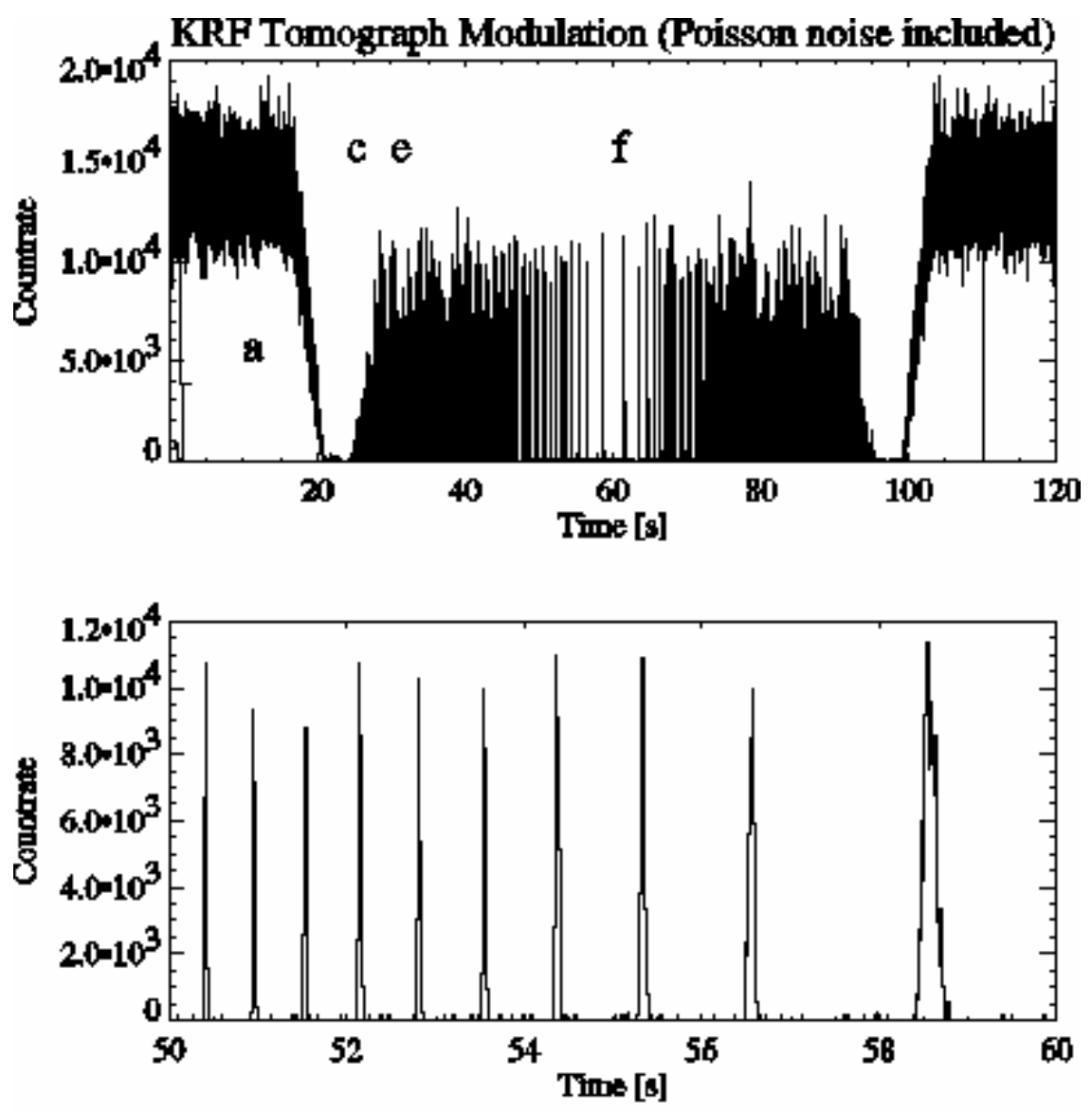

Figure 27: Example of the RMC signal from a simple conical source of 10 arcsec FWHM in case when the rotation axis of the satellite is pointed $\delta=6^{\circ}$ off the source. In the upper panel individual phases of rotation are designated. 


\subsubsection{Performance Parameters of Existing Systems}

Three existing systems are compared. All were designed for space-based x-ray and $\gamma$-ray astronomy.

1. RHESSI (Reuven Ramaty High Energy Solar Spectroscopic Imager).

RHESSI is a satellite-based experiment, currently in use, that is imaging the Sun with an emphasis on studying Solar-flares. The instrument consists of a $\mathrm{RMC}$ in front of nine germanium coaxial detectors. The RMC actually consists of many sets of subcollimators to give a range of performance across energies and field of view. The two grids are separated by $1.5 \mathrm{~m}$ and the pitch of the subcollimators ranges from $0.034 \mathrm{~mm}$ to $2.754 \mathrm{~mm}$ [58].

2. Hinotori: Hinotori was a satellite experiment launched in 1981 by the Institute of Space and Astronautical Science (Japan). It flew for 10 years and had the purpose of imaging solar flares as well as observing x-ray bursts.

3. GRID (Gamma-Ray Imaging Device). GRID was a proposed balloon-based instrument proposed for hard $\mathrm{x}$-ray and $\gamma$-ray imaging. It is a variation of the RMC based on Fourier transform imaging. Like RHESSI, GRID consists of many subcollimators (32) to give a range of resolution from acrseconds to arcminutes. Instead of rotating, like the RMC, this system used "pseudorandom" jitter to produce that modulation. That is, the normal instrument pointing was not controlled with precision. However, the point was known (measured) with great precision, allowing for reconstruction of the source image [61].

4. Modulation Collimator: The modulation collimator is another variation of the RMC that was built and tested in the lab. It was based on $\mathrm{NaI}$ detectors and had a relatively large field of view $\left(27^{\circ}\right)$ [62]. 


\begin{tabular}{|c|c|c|c|c|}
\hline & RHESSI & GRID & $\begin{array}{l}\text { Modulation } \\
\text { Collimator }\end{array}$ & Hinotori \\
\hline $\begin{array}{l}\text { Imaging } \\
\text { Dimensions }\end{array}$ & $2-D$ & $2-D$ & $2-D$ & $2-\mathrm{D}$ \\
\hline Weight & $\begin{array}{l}\text { Heavy: tungsten } \\
\text { collimator }\end{array}$ & $\begin{array}{l}\text { Moderate: } \\
\text { tungsten and } \\
\text { tantalum } \\
\text { collimator }\end{array}$ & Moderate & Heavy \\
\hline Energy Range & $3 \mathrm{keV}$ to $20 \mathrm{MeV}$ & & $10 \mathrm{keV}$ to $100 \mathrm{keV}$ & $<50 \mathrm{keV}$ \\
\hline Energy Resolution & $2 \mathrm{keV}$ to $5 \mathrm{keV}$ & $\begin{array}{l}\text { Moderate } \\
\text { (scintillation } \\
\text { counters) }\end{array}$ & $\begin{array}{l}\text { Moderate } \\
\text { (Nal detector) }\end{array}$ & \\
\hline \multicolumn{5}{|l|}{ Angular Resolution } \\
\hline $3 \mathrm{keV}$ to $400 \mathrm{keV}$ & $2-7$ arcsec & 1.6 arcsec & $1^{\circ}$ & 2.8 arcmin \\
\hline $400 \mathrm{keV}$ to $20 \mathrm{MeV}$ & $\begin{array}{l}2 \text { arcsec to } 3 \\
\text { arcmin }\end{array}$ & $\sim$ few arcmin & -- & \\
\hline Field of View & $1^{\circ}$ to $7.5^{0}$ & $1^{0}$ (full Sun) & $27^{\circ}$ & \\
\hline Efficiency & $<50 \%$ & & $37 \%$ & \\
\hline Detector Area & $350 \mathrm{~cm}^{2}$ & $480 \mathrm{~cm}^{2}$ & $200 \mathrm{~cm}^{2}$ & \\
\hline
\end{tabular}

Table 2: Characteristics of RMC imaging systems. 


\subsection{Compton imagers}

As the last concept, which will be discussed in more detail in this report, Compton imaging promises to improve some of the aforementioned capabilities if properly implemented. In the following, we will briefly discuss the basic concept of Compton imaging and some potential capabilities. We will then discuss a variety of implementations, which are being pursued by different groups in the U.S. and elsewhere. Currently, Compton imaging is driven by astrophysics and Homeland Security applications. Only a small fraction of the worldwide effort is currently dedicated to biomedical imaging which might change in the near future if the currently being developed systems can demonstrate the gain in sensitivity also in this type of applications.

\subsubsection{Concept of Compton imaging}

As first published by Todd for nuclear medicine [23] and Schönfelder for astrophysics [24], the concept of Compton imaging relies on the Compton scattering process and the relationship between the scattering angle $\theta$, the energy of the incident gamma ray $\mathrm{E}_{\gamma}$, and the energy of the first interaction $\mathrm{E}_{1}$ :

$$
\cos (\theta)=1+\frac{511}{E_{\gamma}}-\frac{511}{E_{\gamma}^{\prime}} ; \quad E_{\gamma}^{\prime}=E_{\gamma}-E_{1}
$$

In contrast to conventional and collimator-based gamma-ray imaging systems, this approach requires an incident gamma ray to interact at least twice in the detector: one Compton scattering and one photo electric absorption ${ }^{4}$. While in conventional imaging system this event type can lead to a degradation of the system response, here it is a requirement and the instrument has to be designed accordingly. As illustrated in Figure 27 and Figure 28, the scattering angle describes a cone whose symmetry axis is defined by the line connecting the positions of the first two interactions and the apex lies at the location of the first interaction. For far-field imaging, the projection of those cones on a sphere will overlap at the source location when many events are imaged or backprojected.

\footnotetext{
${ }^{4}$ However, if the gamma-ray energy is known a priori two Compton scatter interactions are sufficient for Compton imaging; full absorption is not required.
} 
Figure 26: The principle of Compton imaging. By measuring the position and energy of each photon interaction, the initial photon direction can be determined thriough the Compton scatter formula to within an annulus (event circle) on the sky.

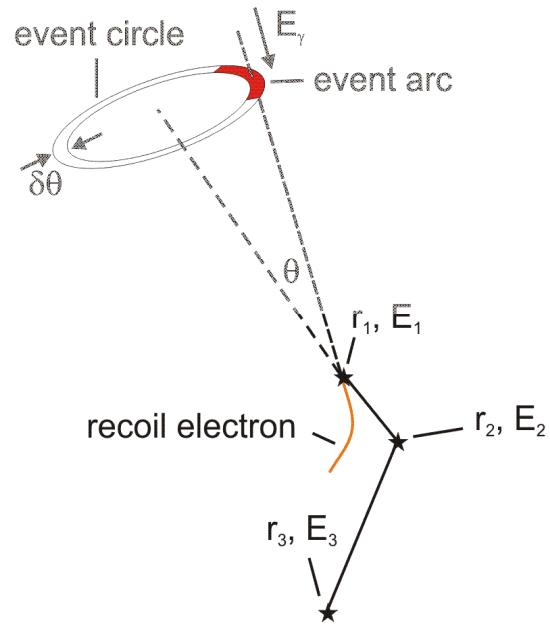

Without measuring the direction of the Compton electron the incident angle of the gamma ray can only be determined to be on a cone surface. Since in semi conductors, the range of electrons is typically less than $1 \mathrm{~mm}$ (for instance, an electron of about $500 \mathrm{keV}$ has a range of about $0.5 \mathrm{~mm}$ in $\mathrm{Ge}$ ) it is very difficult to measure the scattering angle of the electron, particularly considering the complex slowing-down process of electrons. Only in low-Z or low-density detectors such as gases at a pressure of about $1 \mathrm{~atm}$, can electron vertices be measured [18]. However, the efficiency to induce a gamma-ray interaction at all in such instruments is extremely low and therefore the overall sensitivity is very small, too.

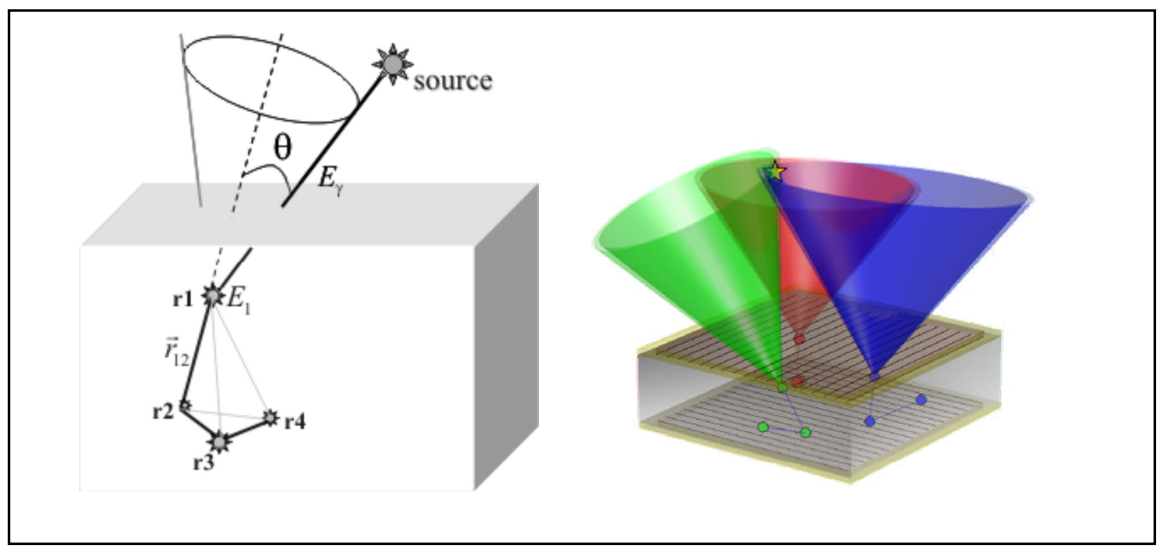

Figure 27: Illustration of Compton imaging principle. The positions of the first two interactions define the symmetry axis of a cone whose opening angel is defined by the energy of the first interaction and the total gamma-ray energy.

As it can be seen on the right of Figure 28, three cones overlap in one point, which enables the determination of the source location in three dimensions and therefore is able to provide tomographic information. However, this tomography with a single detector only works if the source is sufficiently close to the detector (near-field imaging). 
Otherwise, the cones are projected on a plane or a sphere and for every event we obtain a ring. In contrast to collimator-based systems, each event therefore does not only contribute an increment to a single pixel - representing the source location - but a ring. This ring effect degrades the overall signal-to-background and has to be taken into account. It is important to note that this ring effect degrades the signal-to-background not only because the gamma rays from the source of interest induce more background in $4 \pi$ but also background events from different locations induce background in the source location. This feature is similar to a feature, which is found in energy spectra where gamma rays only leave a partial energy in the detector before they escape the detector e.g. after Compton scattering. These Compton-escape events induce the so-called Compton background at lower energies and therefore degrade the peak-to-background ratio in the energy spectrum. As for the energy spectrum, the Compton imaging response can be taken into account for image reconstruction to improve resolution and contrast, e.g. to improve the ability to visually identify sources as it is done for other gamma-ray imaging modalities, particularly in single-photon or position emission tomography (SPECT or PET, respectively). Here, image reconstruction is crucial to obtain meaningful images in three dimensions. In Compton imaging, image reconstruction is crucial in two dimensions already. While a variety of image reconstruction methods have been developed and are being used for SPECT and PET, for Compton imaging no advanced reconstruction methods have been established. This is due to the fact that until recently, no efficient Compton cameras could be built and image reconstruction for Compton imaging is very complex. Only recently, a fast filtered-backprojection algorithm was developed for far-field Compton imaging [63].

The angular resolution that can be obtained in Compton imaging depends on the uncertainties in the determination of the positions of the interactions and the deposited energies. The error in the positions affects the cone axis direction while the error in the energies affects the angle $\theta$ of the cone. The position and energy resolution can in principle be improved for better angular resolution but the electron on which the photon Compton scatters carries momentum. This Doppler broadening effect is unknown a priori and will limit the best angular resolution attainable [64-67]. The effect depends on gamma ray energy, scattering angle, and the $\mathrm{Z}$ of the material. It is smaller for higher energies, forward scattering, and low $\mathrm{Z}$ materials.

\subsubsection{Gamma-ray tracking}

One important requirement to provide high-sensitivity Compton imaging is the ability to time order individual gamma-ray interactions. While position-sensitive gamma-ray detectors are able to provide energies and three-dimensional positions of these interactions, the time resolution of the detectors is generally not sufficient to time order the interactions by the time difference ${ }^{5}$. Of course, by separating the interactions by several meters as it was done in COMPTEL the time resolution would be sufficient,

\footnotetext{
${ }^{5}$ Assuming $10 \mathrm{~cm}$ as separation of two gamma-ray interactions, the time difference between them is about $0.3 \mathrm{~ns}$ which is about a factor 10 less than the time resolution of the gamma ray detectors which can be used for gamma-ray imaging.
} 
however, the solid angle coverage to detect the interactions will be dramatically reduced, too. The alternative to the direct time measurement is the so-called gamma-ray tracking: Using the relationship between energies and scattering angles given by the Compton formula and comparing them to the experimentally obtained values, one is able to get the most likely scattering sequence. However, not only is it possible to deduce the scattering sequence of the gamma ray but to distinguish between full-energy and escape events. In this way, the spectroscopic response can be improved by increasing the peak-tobackground ratio in the energy spectrum. In addition, observing three or more interactions enables to reconstruct the full gamma ray energy even if the full energy was not observed and the gamma ray escaped. However, the efficiency and the accuracy in reconstructing the full energy and the incident angle are limited.

\subsubsection{Compton imaging to maximize sensitivity: "Every photon counts"|"Leave no photon behind"}

The fact that Compton imaging systems are operated without a collimator and can accept all gamma rays from all directions, in addition to having the ability to track gamma rays by measuring individual gamma-ray interactions with high accuracy, provides the foundation to enhance the sensitivity in finding and identifying gamma-ray sources. The ability to track gamma rays provides a new capability to distinguish between Comptonescape and full-energy events to improve the signal-to-background in the energy or the image. It also potentially enables reconstruction of the full energy of the gamma even if only a fraction of the total energy was deposited. Finally, one has the imaging capability, which is a new degree of freedom, to separate a signal from the background. Overall, such a gamma-ray tracking based Compton imager can be seen as one step towards the ultimate gamma-ray detector.

To estimate the gain in sensitivity of a Compton imaging system over more conventional imaging systems and non-imaging systems we introduce the following sensitivity measure S:

$$
\begin{array}{cc}
S^{2} \sim A \times \frac{E}{\Delta E} \times \varepsilon_{\text {int rinsic }} & \text { (conventional detector) } \\
S^{2} \sim A \times \frac{E}{\Delta E} \times \varepsilon_{\text {int rinsic }} \times \varepsilon_{\text {image }} \times \frac{4 \pi}{\Delta \Omega} & \text { (imaging detector) }
\end{array}
$$

Here, $\mathrm{A}$ is the area of the detector, $\Delta \mathrm{E} / \mathrm{E}$ the relative energy resolution at the energy $\mathrm{E}$, $\varepsilon_{\text {intrinsic }}=\varepsilon_{\text {intrinsic }}(\mathrm{E})$ is the intrinsic efficiency to detect the full energy of the gamma ray of energy $\mathrm{E}, \varepsilon_{\text {image }}=\varepsilon_{\text {image }}(\mathrm{E}, \Delta \Omega, \Delta \mathrm{E})$ the imaging efficiency, and $\Delta \Omega / 4 \pi$ the relative angular resolution. It is important to point out that the imaging efficiency can depend on the energy of the incident gamma ray, the energy resolution of the instrument and the angular resolution. The fact that the efficiency is inverse proportional to the angular resolution reflects the trade-off between both parameters. For example, the angular 
resolution can be improved by separating the first two interactions more, e.g. by moving detectors further apart or by using lower pressure in a gas detector. However, the drawback is the reduced overall efficiency since the solid angle coverage is reduced or the interaction cross section is reduced, respectively. Figure 28 illustrates the potential gain of a compact Compton imaging system consisting of a Si detector in front of a $\mathrm{Ge}$ detector.

The plot shows the angular resolving power $\varepsilon_{\text {image }} \times \varepsilon_{\text {int rinsic }} \times 4 \pi / \Delta \theta$, which takes into account the intrinsic and imaging efficiencies and the angular resolution $\Delta \theta$. The angular resolving power is plotted as a function of the angular resolution for 5 different energies, which are of interest, e.g. for homeland security applications. Efficiencies and angular resolution were calculated for a Compton imaging detection system, which consists of a planar Si detector with an area of $5 \times 5 \mathrm{~cm}^{2}$ and a thickness of $2 \mathrm{~cm}$ in front of a planar Ge detector with an area of $8 \times 8 \mathrm{~cm}^{2}$ and a thickness of $4 \mathrm{~cm}$. Such an implementation is currently being used by us at LLNL and represents one example of other implementations. However, the implementation with high-resolution, and positionsensitive Si and Ge detectors, both operated at LN temperatures provides the best currently achievable sensitivity for Compton imaging. Such a system can be built out of two double-sided strip detectors (DSSD) made of Li-drifted silicon ( $\mathrm{Si}(\mathrm{Li})$ ) and two highpurity germanium (HPGe) detectors also in DSSD configuration. To obtain the results shown in Figure 29, realistic energy resolution values for $\mathrm{Si}(\mathrm{Li})$ and HPGe detectors and a position resolution of $1 \mathrm{~mm}$ was assumed. The Doppler broadening effect which impacts the ultimate resolution, which can be achieved in Compton-scatter based imaging instruments was taken into account. Conventional gamma-ray imaging systems such as a pinhole system at $186 \mathrm{keV}$ and a parallel-hole system at $186 \mathrm{keV}$ and $375 \mathrm{keV}$ are also plotted [2]. The latter is shown for three different collimator configurations, which are widely being used for medical applications. A few points are noteworthy here: first, the sensitivity of a Compton imaging system does not depend too strongly on the incident gamma-ray energy, in strong contrast to collimator-based systems. Secondly, the lowest sensitivity is achieved for small and high energies. The low-energy sensitivity is limited by the imaging efficiency, i.e. the requirement that at least two interactions need to occur and that these two interactions must be well separated and measured with high accuracy. 


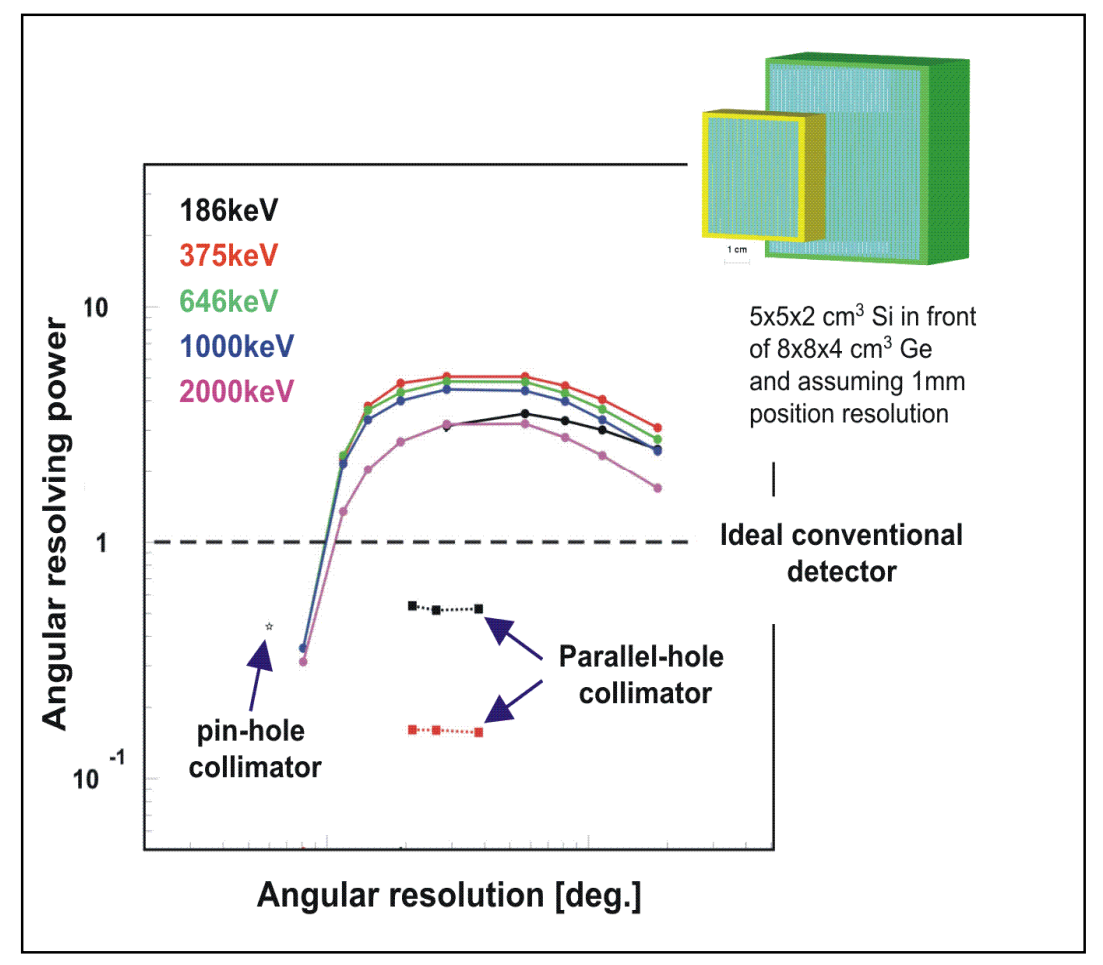

Figure 28: Gain in sensitivity of a hybrid Compton imaging built of position -sensitive Si and Ge detectors in comparison with parallel-hole and pinhole systems and an ideal conventional, non-imaging detector. Plotted is the gain in sensitivity as function of angular resolution.

For example, in Ge the photoelectric effect is dominant up to about $150 \mathrm{keV}$ and Compton scattering below this energy is very unlikely. But even above $150 \mathrm{keV}$ it is very difficult to identify these two interactions and measure their positions sufficiently accurately to obtain a good angular resolution. For example, assuming a gamma-ray energy of $300 \mathrm{keV}$, on average Compton scattering leads to an energy deposition of 150 $\mathrm{keV}$ and a scattered photon of $150 \mathrm{keV}$. This $150 \mathrm{keV}$ scattered photon will typically be absorbed in Ge within $2 \mathrm{~mm}$. Even if these two interactions can be distinguished, the position resolution needs to be much better than $1 \mathrm{~mm}$ to obtain an angular resolution of better than $10 \mathrm{deg}$. In addition, at low energy energies the Compton profile degrades the achievable resolution. The sensitivity for higher gamma-ray energies is limited by the detection efficiency as in other non-imaging instruments due to the reduced attenuation of gamma rays at higher energies.

These aspects need to be taken into account by designing a Compton imaging system to provide maximum sensitivity between $150 \mathrm{keV}$ and $3 \mathrm{MeV}$.

\subsubsection{Compton imager configurations}

As for conventional and non-imaging gamma-ray detection systems, Compton imaging systems can be built of a variety of materials and configurations. Following the 
discussion from above, a large system built of low- $Z$ and low-density material would be ideal to optimize the imaging efficiency due to their ability to induce multiple interactions, which are well separated, and can thus easily be distinguished and the positions measured accurately to obtain high angular resolution. In addition, the low-Z material minimizes the Doppler broadening at low energies. Such a system can be envisioned either built of a gas such as Neon or Argon or Si. In particular, low-Z and low-density gas detectors have the potential to provide not only the position of the Compton interaction but the track of the Compton-scattered electron which enables the incident direction of the gamma ray to be determined and not just the angle which results in a cone. However, to achieve sufficient detection efficiencies at high gamma-ray energies these systems solely built of low-Z and low-density materials need to big, typically in the order of $0.5-1 \mathrm{~m}^{3}$. A Ne-based time-projection chamber, which was used to measure the path of the photoelectron from a $5.5 \mathrm{keV}$ X-ray, was operated at $1 \mathrm{~atm}$ [18]. It is important to remember that $1 \mathrm{~atm}$ of $\mathrm{Ne}$ has less attenuation than air, that means the attenuation and therefore the detection efficiency is extremely low (the attenuation length for $186 \mathrm{keV}$ in air is about $70 \mathrm{~m}$ !). Gas detectors can be operated up to $50 \mathrm{~atm}$ which produces densities of $1-2 \mathrm{~g} / \mathrm{cm}^{3}$ and are therefore almost as high as solid state detectors, however, the potential benefit of measuring the electron path is not achievable at these pressure values and the operation of a large volume at $50 \mathrm{~atm}$ is extremely challenging. Also, the energy resolution degrades significantly at high pressure limiting the Compton imaging capability to a minimum [68, 69].

An alternate approach is the use of position-sensitive Si detectors as proposed by the Naval Research Laboratory [70]. As for gas, the use of low-Z material exclusively is appealing for Compton imaging, the requirements of sufficient detection and high imaging efficiency can only be met with $>0.5 \mathrm{~m}^{3}$ large detectors with $>10^{6}$ channels which is also extremely challenging. In addition, such a system is not scalable since a large volume is required to obtain a finite detection efficiency. One way to reduce the volume requirement is the implementation of gamma-ray tracking to reconstruct the gamma-ray path, the full gamma-ray energy, and eventually the incident angle of the gamma ray even if the energy was not fully deposited in the instrument. While this approach is appealing it requires at least three interactions in the instrument and it degrades the energy and image resolution significantly.

A more practical and also scalable approach relies on a hybrid system made of a low-Z and a higher- $Z$ detection material as introduced in the previous section. This approach which promises the highest sensitivity which is currently attainable with a scalable system consists of high-energy resolution, position-sensitive, and large $\mathrm{Si}(\mathrm{Li})$ and HPGe detectors. The excellent energy resolution of these detectors provide both, important prerequisites for isotope identification and high-sensitivity Compton imaging. The drawback in this approach is the requirement to operate these detectors at liquid nitrogen temperatures, which impacts operational considerations. However, for demonstration purposes of Compton imaging this is the best approach. One can also envision a roomtemperature operated hybrid instrument made of intrinsic Si detectors and pixelated $\mathrm{CdZnTe}$ detectors. Such a system is not able to provide comparable energy resolution and can only built with thousands of channels, even for a small-scale system, which requires sophisticated integrated electronics. However, the ability to operate such a system 
without cryogens and associated cryostats and cooling will allow more compact systems, even pocket-sized systems.

\subsubsection{Compton imaging systems}

In the following we will briefly discuss several different possible implementations, which are currently being pursued in the U.S. and world-wide.

\subsubsection{COMPTEL}

The COMPTEL system represents the first and so far only Compton imaging instrument, which was developed and deployed for full operation $[12,13]$. It was a space-based instrument mounted on the CGRO satellite and flown in the 90's. It provided new insights in the structure and distribution of gamma-ray sources in the sky in an energy range from $500 \mathrm{keV}$ to $30 \mathrm{MeV}$. The COMPTEL instrument consists of two parallel arrays of $\mathrm{NaI}(\mathrm{Tl})$ detectors which are separated by several meters. This separation was necessary to identify the first and second interactions through the time difference of the interactions. The $\mathrm{NaI}(\mathrm{Tl})$ detectors used were not able

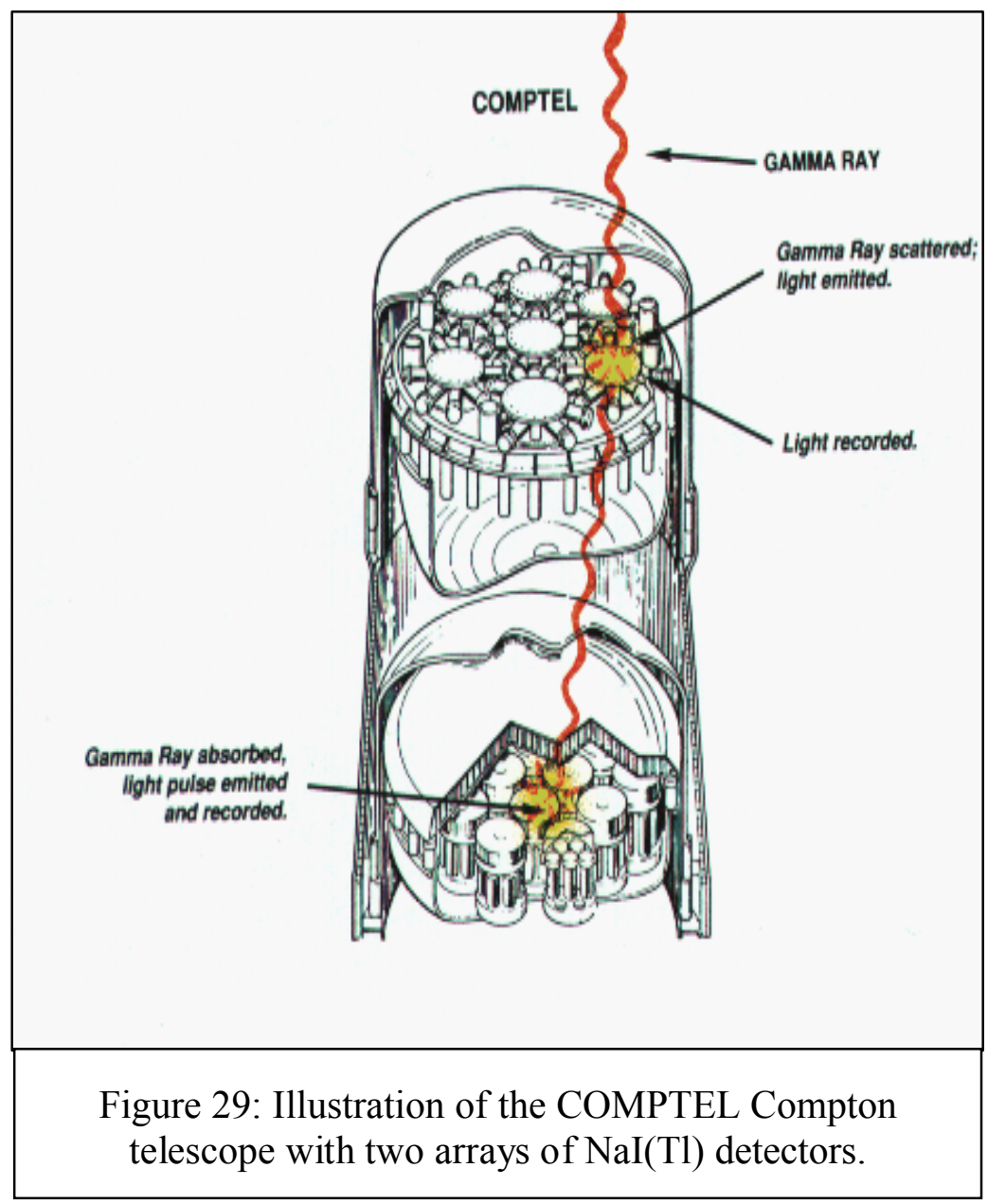
to provide sufficient position and energy resolution to provide the scattering sequence by gamma-ray tracking. At $1 \mathrm{MeV}$, the obtained relative energy resolution, angular resolution and FOV was in the order of $10 \%, 10 \mathrm{deg}$ and $0.3 \pi$, respectively. The overall sensitivity was very low with $10^{-5}$ at $1 \mathrm{MeV}$ primarily due to the large distance between the to detector plans. 


\subsubsection{CSPRINT}

While COMPTEL was developed and deployed as a fully operational instrument for astrophysics applications, C-SPRINT was developed for biomedical applications at the University of Michigan but was only used for demonstration purposes and never as a fully operational instrument [7]. It was used as an evaluation tool primarily in the 90's to demonstrate the potential and the limitations of Compton imaging for biomedical applications, e.g. for SPECT as an alternative to standard Anger cameras. Figure 31 illustrates the setup of C-SPRINT. It consists of a pixellated Si detectors in front of an array of $10 \mathrm{~cm}$ long $\mathrm{NaI}(\mathrm{Tl})$ detectors arranged in a ring with a diameter of $50 \mathrm{~cm}$ to cover the full azimuthal angle and a scattering angle between about 100 and $115 \mathrm{deg}$. The silicon "pad" detector module was $3 \times 3 \times 0.1 \mathrm{~cm}^{3}$ and was pixellated into a 22 by 22 array of 1.2 by 1.2 $\mathrm{mm}^{2}$ elements. The advantage of these pixellated detectors is the good energy and position resolution of about $1 \mathrm{keV}$ and 1.2 $\mathrm{mm}$, which can be achieved, respectively. In addition, high total count rates can be achieved due to the use of small pixels. The $\mathrm{NaI}(\mathrm{Tl})$ system were arranged in 11 modules, with each module consisting of an array of $1.27 \mathrm{~cm}$ thick detectors viewed by an array of

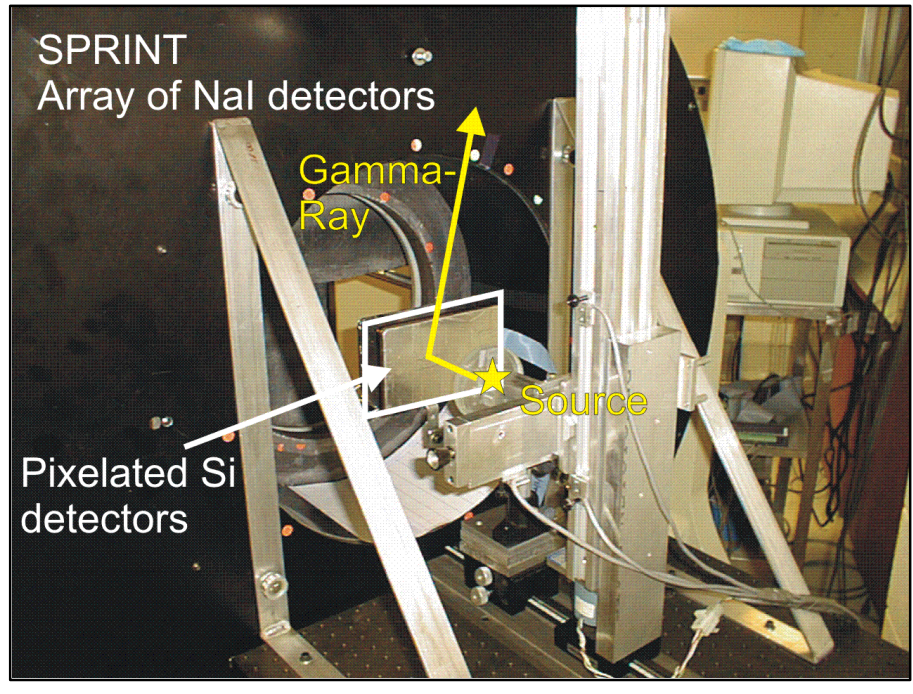

Figure 30: Picture of the CSPRINT system. It consists of pixilated Si detectors in front of the SPRINT camera which consists of an circular array of $\mathrm{NaI}(\mathrm{Tl})$ detectors originally built for SPECT applications.

photomultiplier tubes that provide $3 \mathrm{~mm}$ intrinsic spatial resolution at $140 \mathrm{keV}$. The overall Compton efficiency for this setup was estimated to be $2.7 \times 10^{-5}$. By increasing the Si detector dimensions to $9 \times 9 \times 0.5 \mathrm{~cm}^{3}$ and using $30 \mathrm{~cm}$ long NaI(Tl) crystals in a cylinder radius of $12.5 \mathrm{~cm}$ it was calculated that the efficiency can be increased to $2.7 \times 10^{-3}$. We want to point here that with our approach of two $10 \mathrm{~mm}$ thick $\mathrm{Si}(\mathrm{Li})$ in front of two $15 \mathrm{~mm}$ thick HPGe detectors, the Compton imaging efficiency can be improved another order of magnitude.

Employing the previously discussed UCR lower bound estimate, it was shown that the enhanced C-SPRINT system is required to match the performance of the parallel-hole system at $140 \mathrm{keV}\left({ }^{99 \mathrm{~m}} \mathrm{Tc}\right)$ while the original C-SPRINT system as shown in Figure 31 is already comparable at $364 \mathrm{keV}\left({ }^{131} \mathrm{I}\right)$. Therefore, the sensitivity of this still not optimized system at this or higher energies can be increased significantly over the collimator-based system. 


\subsubsection{LXeGRIT imager}

The Liquid Xenon Time Projection Chamber Gamma-Ray Imaging Telescope (LXeGRIT) was primarily developed by Columbia University in the 90's and deployed and demonstrated as a small imager on balloon missions for astrophysics applications [71]. Figure 32 shows a schematic drawing and pictures of the actual instrumental chamber.

LXeGRIT is based on liquid Xe and operated as a Time-Projection Chamber (TPC). The $\mathrm{Xe}$ ionization and the scintillation is used to determine energy and three-dimensional positions of individual interactions. The scintillation is used to measure the total energy of the gamma ray and it provides the start time for the charge carrier drift towards a twodimensional position sensitive detector consisting of orthogonally arranged wires. The drift time of the charge carriers from the start by the scintillation to the charge collection at the wire determines the third dimensions of the interactions. The system flown on a balloon consisted of a $20 \times 20 \mathrm{~cm}^{2}$ sensitive area and $7 \mathrm{~cm}$ drift depth with about $1 \mathrm{~mm}$ position resolution in all three dimensions. An electrical field $1 \mathrm{kV}$ was applied which
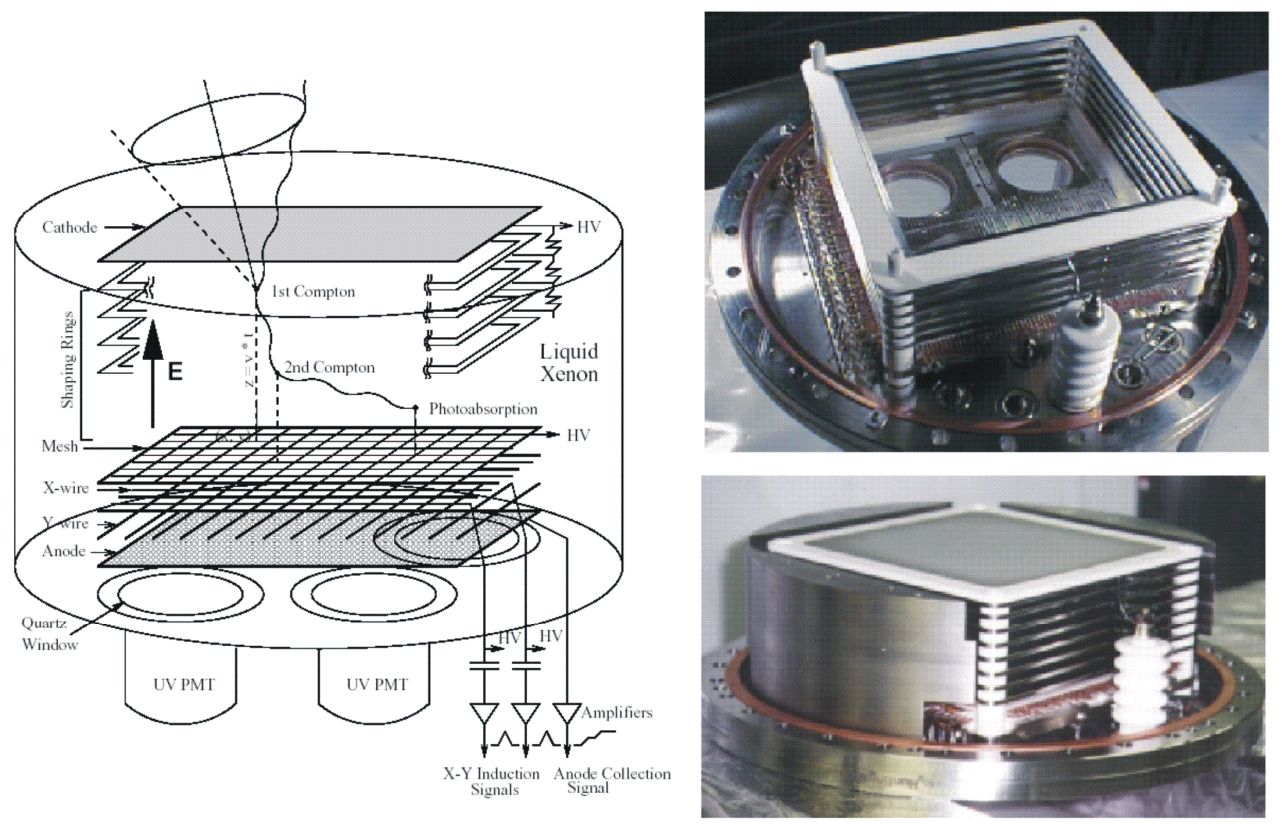

Figure 31: Illustration of LXeGRIT principle (left) and pictures of actual system (right).

resulted in a collection time of up to $35 \mu \mathrm{s}$. The overall dimension of this system was a cylinder with a diameter of $50 \mathrm{~cm}$ and a height of $35 \mathrm{~cm}$ and weighted $190 \mathrm{~kg}$ including $24 \mathrm{~kg}$ for liquid $\mathrm{Xe}$. The energy resolution was about $10 \%$ at $1 \mathrm{MeV}$, the angular resolution was determined to be 3 deg at $1.8 \mathrm{MeV}$. The FOV was 2 sr (FWHM). The overall mass of the total instrument was $2000 \mathrm{lbs}$ with $450 \mathrm{~W}$ power consumption. 
While the approach of liquid Xe appears appealing due to its potential scalability, significant challenges remain to achieve adequate energy resolution and efficiency. Due to the use of $\mathrm{Xe}$ with $\mathrm{Z}=54$, imaging below $500 \mathrm{keV}$ will be difficult. In addition, significant amounts of additional weight and equipment is required to operate a liquid $\mathrm{Xe}$ (or Ar) detector due to the high pressure and low temperature to keep the material liquefied.

\subsubsection{MEGA/TIGRE imager}

The Tracking and Imaging Gamma-Ray Experiment (TIGRE) for balloon flight observations is an effort, which is led by UC Riverside in California [72]. The overall approach of using many layers of $0.3 \mathrm{~mm}$ thick DSSD HPSi detectors embedded in an array of $\mathrm{CsI}(\mathrm{Tl})$ detectors is very similar to the original approach of MEGA which was led by MPI of Exterrestrial Physics in Munich before further funding was withdrawn [73]. MEGA was supposed to become the German successor of COMPTEL. Figure 33

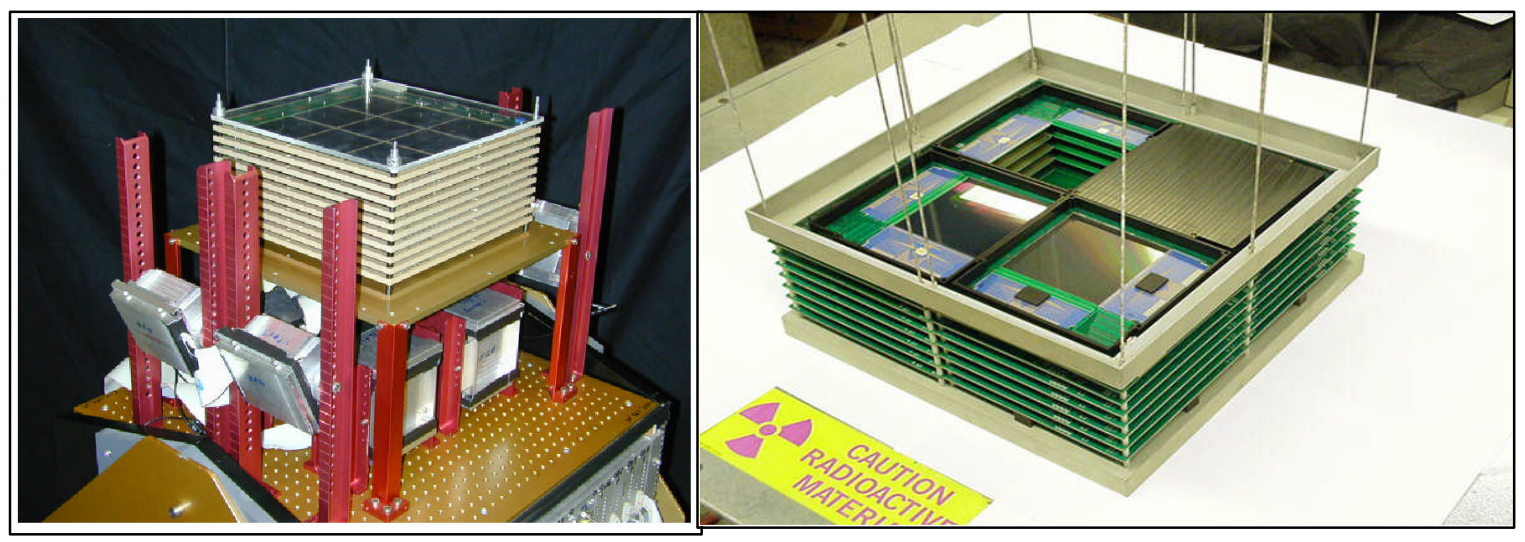

Figure 32: Photograph of MEGA (left) and TIGRE (right) with the arrays of stacked DSSD HPSi detectors and components. On the left several of the CsI(Tl) detectors can be seen as well.

shows MEGA and TIGRE systems and system components.

The TIGRE collaboration is still pursuing an approach employing an array of $0.3 \mathrm{~mm}$ thick and room-temperature operated HPSi in combination with pixilated CsI detectors for astrophysics applications. The proposed instrument is shown in Figure 34. The goal is to assemble 64 layers of $2 \times 2$ arrays of $10 \mathrm{~cm} \times 10 \mathrm{~cm} \times 0.3 \mathrm{~mm}$ DSSD HPSi detectors with a pitch size of about $0.75 \mathrm{~mm}$ resulting in 128 strip channels per side per detector. Therefore, per $10 \times 10 \mathrm{~cm}^{2}$ component $16 \mathrm{k}$ channels need to be readout and in total $64 \mathrm{k}$ just for the Si. The first, smaller-scale system is readout by IDEAS ASIC components which achieves an energy resolution of about $10 \mathrm{keV}$ (FWHM) at $122 \mathrm{keV}$. Monte-Carlo simulations indicate that an angular resolution of $2 \mathrm{deg}(1 \sigma)$ is achievable at $1 \mathrm{MeV}$ limited by the energy resolution of the $\mathrm{CsI}(\mathrm{Tl})$ detectors which is about $6 \%$ (FWHM) at 1 $\mathrm{MeV}$. 


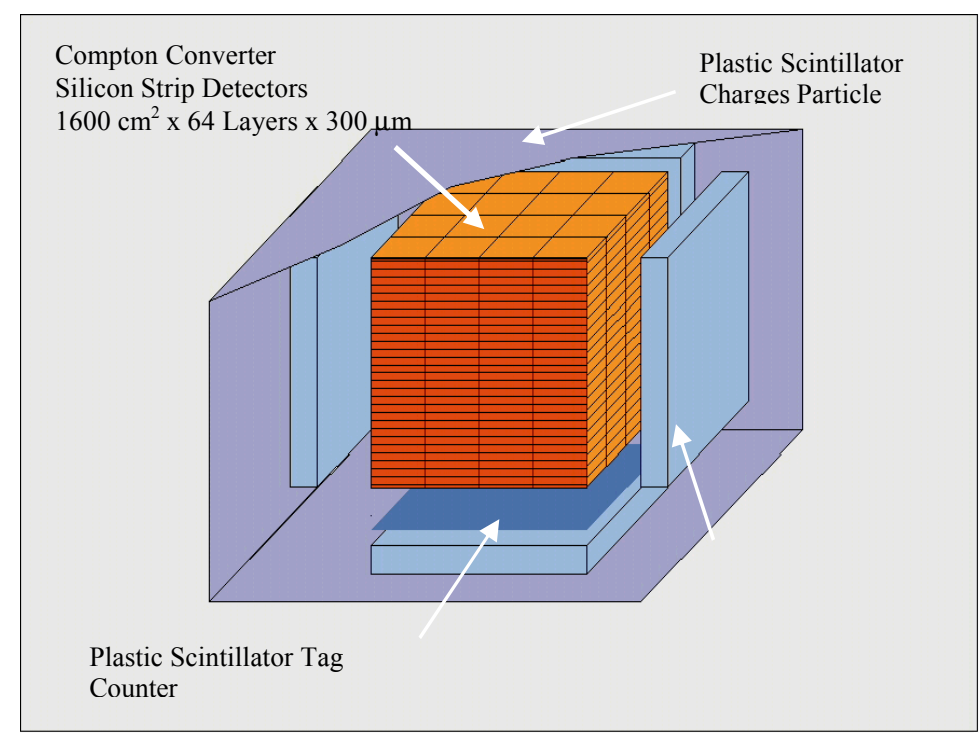

Figure 33: Schematic illustration of the TIGRE instrument as proposed.

The goal of the MEGA collaboration was similar with $2.4 \mathrm{deg}$ angular resolution (FWHM) and 3\% energy resolution (FWHM), both at $2 \mathrm{MeV}$.

The TIGRE collaboration also realizes that with an energy resolution of $2 \mathrm{keV}$ as it can be achieved with a Ge detector and a position resolution of $1 \mathrm{~mm}$, an angular resolution of $<0.5$ deg can be achieved at $1 \mathrm{MeV}$.

The TIGRE collaboration was able to demonstrate electron tracking down to $800 \mathrm{keV}$ with the array of thin HPSi detectors, however, the efficiency for these events was very small, the resolution was very limited and therefore the overall gain in sensitivity due to electron tracking was found to be negligible below $1 \mathrm{MeV}$.

\subsubsection{5 $\operatorname{Ar}(X e)+C s /$ imager}

Ar+CsI [74]: A combination of gaseous Ar or Xe as scatterer and CsI as catcher is being pursued by the Univ. Kyoto group, for astrophysics as well as medical applications. Electron tracking has been demonstrated for gamma rays at $662 \mathrm{keV}$. However, to enable the tracking of the electron the small volume of Ar gas was operated at a pressure of 1 atm, which resulted in an extremely small overall efficiency $\left(<10^{-6}\right)$. In addition, due to the limited energy resolution the angular resolution was only about 20 degrees.

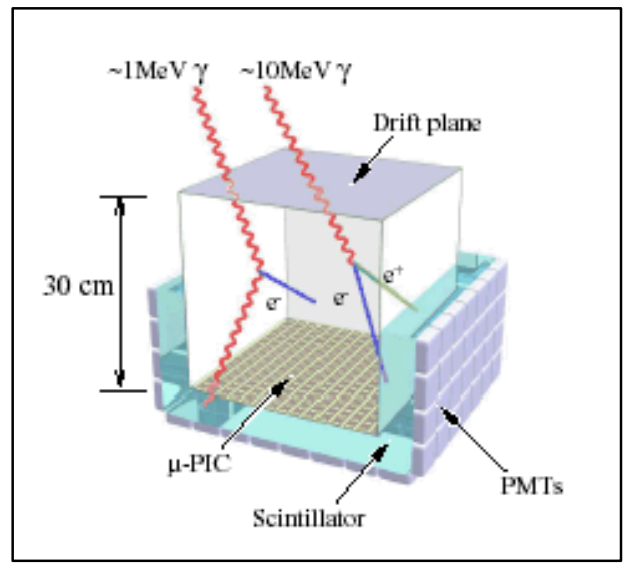

Figure 34: Schematic view of proposed Ar+CsI Compton imager with an Ar gas chamber operated as micro-TPC surrounded by position sensitive scintillators. 


\subsubsection{Si+CdTe imager}

HPSi+CdTe [75]: This approach is being pursued by a US/Japanese collaboration as a potential gamma ray telescope for the NEXT mission. Compton imaging was demonstrated with a small prototype with reasonable energy and angular resolution. To achieve sufficient energy resolution, $0.3 \mathrm{~mm}$ thick $\mathrm{Si}$ and $0.5 \mathrm{~mm}$ thick CdTe detectors are employed which need to be cooled to about $-10^{0} \mathrm{C}$. To achieve comparable efficiency as with a proposed $\mathrm{Si}(\mathrm{Li})+\mathrm{HPGe}$ system, hundreds of layers of detectors are required, even for a small-scale system. In addition, the implementation pursued for NEXT is characterized by a small FOV reflecting the requirements for astrophysics applications.

\subsubsection{NRL-Si only imager}

The approach of using exclusively "thick" (e.g. $2 \mathrm{~mm}$ ) HPSi detectors has been pursued by NRL for many years. Only recently, they were able to assemble a $2 \times 2$ array of 8 layers of $57 \times 57 \times 2 \mathrm{~mm}^{3}$ DSSD HPSi detectors with 64 strips on each side. The assembly is shown in Figure 36.

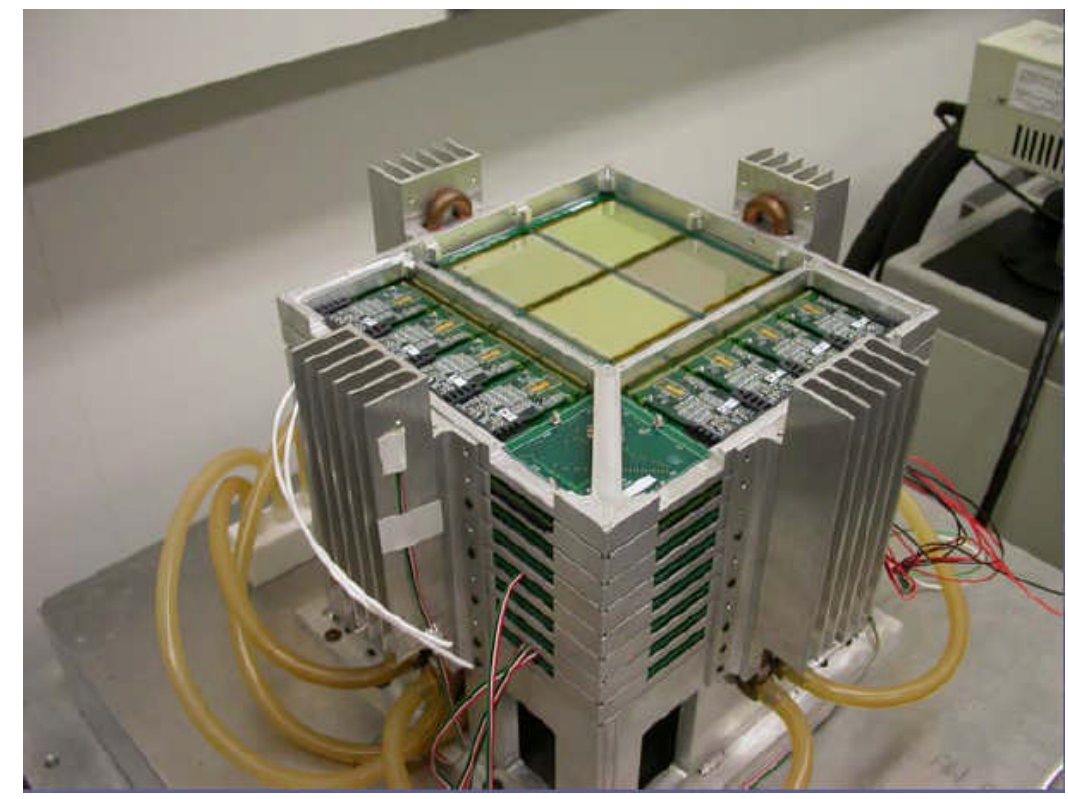

Figure 35: The NRL Si-only Compton imaging instrument with 8 layers of $2 \times 2$ arrays of DSSD HPSi detectors.

Energy resolution values of $4 \mathrm{keV}$ and about $1 \mathrm{keV}$ at $60 \mathrm{keV}$ have been achieved at $25^{\circ}$ $\mathrm{C}$ and $-20^{\circ} \mathrm{C}$, respectively, when operated with discrete electronics. However, when operated with an AISC readout, which is necessary to operate all 4096 channels, the energy resolution degrades to $10-20 \mathrm{keV}$, depending on the side. This instrument is supposed to demonstrate the potential Compton imaging capabilities with a Si-only instrument. The problem is not only the limited energy resolution of $10-20 \mathrm{keV}$ but also the very limited efficiency of this instrument. A total thickness of $16 \mathrm{~mm}$ of Si has only a very small efficiency to fully absorb gamma rays above $100 \mathrm{keV}$. With a subset of this 
Si-only array, the NRL group previously demonstrated the ability to use three and more Compton interactions to reconstruct the full incident gamma-ray energy and the scattering sequence and therefore was able to perform Compton imaging without measuring the full gamma-ray energy [76].

The full gamma-ray energy $\mathrm{E}_{\gamma}$ can be reconstructed using the Compton scattering formula when the energies of the first two interactions $\left(E_{1}, E_{2}\right)$ and the positions of the second and third interaction are measured, the latter providing the scattering angle $\Theta_{2}$ between the second and the third interaction:

$$
E_{\gamma}=E_{1}+\frac{E_{2}+\left[E_{2}^{2}+\frac{4 m_{e} c^{2} E_{2}}{1-\cos \Theta_{2}}\right]^{\frac{1}{2}}}{2}
$$

However, due to this relationship, the energy resolution depends on the energy resolution for the first two interactions and the angular uncertainty $\Delta \Theta_{2}$, which is defined by the position resolution of the second and third interaction and their distance. A total energy resolution of $8 \%$ and $4 \%$ at $1.17 \mathrm{MeV}$ and $1.33 \mathrm{MeV}$ was recently reported [76].

However, in addition to the limited energy resolution, which can be achieved, the overall efficiency is limited, too, since now at least three interactions are required. Monte-Carlo simulations have sown that at $1 \mathrm{MeV}$ about $45 \%$ of all gamma rays interact three or more times but only $27 \%$ of all events interact 3-6 times. Being able to resolve more than 6 interactions in an instrument appears to be an even more difficult task. In addition, with three interactions, the scattering sequence can not be determined uniquely and only with more and more interactions the scattering sequence can be theoretically determined with a probability of $80 \%$. Not being able to reconstruct the scattering sequence properly induces background in the images due to wrong backprojection of the Compton rings.

While the approach of a Si-only Compton imaging instrument is appealing due to the low- $\mathrm{Z}$ and therefore large minimum degradation due to the Doppler broadening and good separation of interactions, the efficiency to fully absorb a gamma ray is very low. The three-Compton method to reconstruct the full energy of the incident gamma ray and to determine the scattering sequence was demonstrated, however, it significantly degrades the performance in terms of energy and angular resolution, efficiency, and signal-tobackground in the image. Only a large-scale system of $1 \times 1 \times 0.5 \mathrm{~m}^{3}$ might be able to provide sufficient efficiency to demonstrate Compton imaging. Even a system of $1 \mathrm{x} 1 \mathrm{x}$ $0.2 \mathrm{~m}^{3}$ as proposed by NRL with 100 layers of $16 \times 16$ arrays of DSSD HPSi detectors will still need to rely on the three-Compton imaging reconstruction with all its deficiencies. It is noteworthy that with the current technology of DSSD HPSi detectors of $2 \mathrm{~mm}$ thickness, more than 3 million electronics channels are required to read out this instrument. Since with the DSSD approach the capacity will remain fairly high, it will be very difficult to reduce the power to lower than $10 \mathrm{~mW}$ per channel resulting in more than $30 \mathrm{~kW}$ to operate this system. The only alternative are larger crystals, e.g. larger thicknesses which require much higher purity of the Si material, similar to HPGe which is not in sight for the near future. Even assuming a factor three increase in thickness the 
power consumption will still be $10 \mathrm{~kW}$. Even hybrid systems of low-Z HPSi detector materials with higher- $Z$ and higher density materials such as $\mathrm{CdZnTe}$ will require the development of high-energy resolution ASIC devices. While ASIC devices for lowcapacity, pixilated detectors such as for CdZnTe has been successfully being developed and demonstrated, for larger capacity detectors such as DSSD HPSi systems, these efforts failed so far.

Large systems of $1 \mathrm{~m}^{3}$ are indeed being built, e.g. for large-scale particle physics experiment at the LHC at CERN or for the NASA GLAST mission [77], however, they are built either for charged particles or gamma-ray energies of more than $30 \mathrm{MeV}$, and not for high-resolution gamma-ray detection below $1 \mathrm{MeV}$ (in addition to the fact that these type of instruments cost several 100's of millions of Dollars).

\subsubsection{CZT-only Compton imager}

The approach of using "large"-volume CdZnTe (or CZT) in a pixellated configuration is being pursued by Univ. Michigan for several years now. Compton imaging at $662 \mathrm{keV}$ was demonstrated in a $15 \times 15 \times 10 \mathrm{~mm}^{3}$ large single CZT crystal, which was pixellated in $11 \times 11$ pixels [78].

The compound semi-conductor CZT has much poorer charge collection properties than for example elemental semi-conductors such as $\mathrm{Si}$ or Ge and holes in particular have a very small mobility-lifetime. Although the band gap of CZT is very appealing because it in principle allows very good energy resolution at room temperature, the charge collection properties limit the achievable performance if simple readout contacts are used. However, it was demonstrated that with specific contact geometries such as coplanar grids [79] or pixellation [80], the limited charge collection properties can not only be compensated but can be used to determine the depth of the interaction by correlating the measured signal amplitude of the collecting pixel to the amplitude of the non-segmented contact. If multiple interactions occur, the timing of the pixel signals relative to the timing of the non-segmented contact can be used to derive the relative depth positions of the interactions.

While the approach of a large array of pixellated CZT detectors is appealing for higher gamma-ray energies, the imaging capabilities below $1 \mathrm{MeV}$ are very limited, worse than Xe due to the higher density of CZT. In addition, this approach requires expensive, high quality crystals and many channels due to the pixilated configuration, which is required to achieve sufficient energy and position resolution. An alternative could be the use of thinner, lower quality and therefore cheaper CZT detectors. The development and demonstration of small ( $2 \times 2 \times 2)$ CZT arrays is currently being pursued with $15 \times 15 \times 10$ $\mathrm{mm}^{3}$ detectors. While the drawback of these devices is the limited angular resolution between $1 \mathrm{MeV}$ they are operated at room temperature - and can therefore being build very compact - and still have better than 1\% energy resolution at $662 \mathrm{keV}$. 


\subsubsection{Ge-only Compton imagers}

In this section we discuss several approaches, which are based exclusively on threedimensional position-sensitive Ge detectors. The three-dimensional position sensitivity is derived by combining two-dimensional segmentation and pulse-shape processing. Two different implementations of Ge detectors have been demonstrated, the planar DSSD and the closed-ended coaxial geometry. The advantage of Ge for a Compton imaging system is the excellent intrinsic energy resolution, the relatively large size for single crystals and the very good position resolution, which can be achieved by combining segmentation and pulse processing. The drawback is the required operation at liquid nitrogen temperatures and the cost of Ge detectors. The Ge-only approach is pursued by several groups world wide, e.g. in the U.K.[81], in Japan [82], and here in the U.S. by NRL [83], the Space Science Laboratory [84], and at LLNL [85,86]. In the following we discuss three approaches in more detail, the coaxial Ge detector Compton imager, the DSSD Ge detector Compton imager, both developed at LLNL, and the Next Compton Telescope (NCT), another astrophysics instrument for balloon flights and led by the Space Science Laboratory (SSL) in Berkeley.

\section{The LLNL coaxial HPGe Compton imager}

The coaxial HPGe detector which was used to demonstrate Compton imaging in this configuration is shown in Figure 37. It is a closed-ended, coaxial HPGe detectors with a diameter of $5 \mathrm{~cm}$ and a length of $7 \mathrm{~cm}$. The coaxial part $(5 \mathrm{~cm})$ of the outside contact is divided in 40 segments as an $8 \times 5$ array with each segment a pie shape with 45 deg opening and $1 \mathrm{~cm}$ depth. Each segment as well the central channel is readout through compact and high-resolution FETs and preamplifiers mounted in the back of the detector as it can be seen on the right of Figure 37.
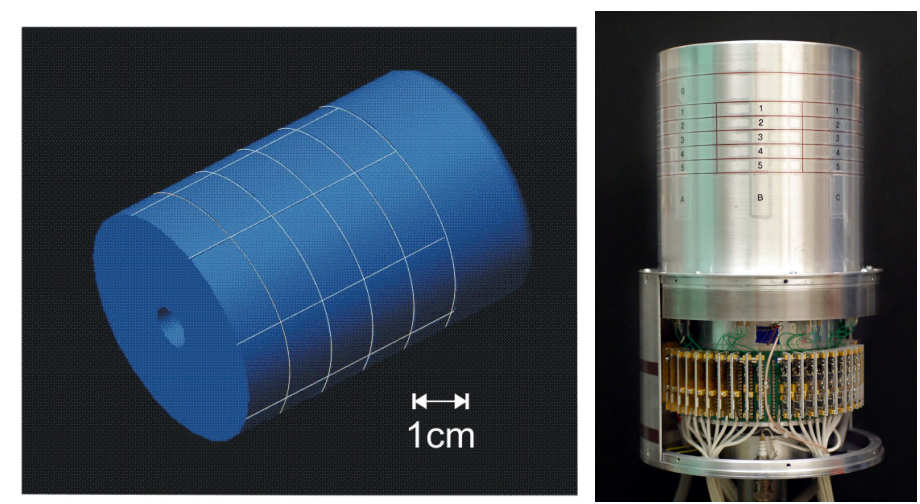

Figure 36: 40-fold segmented, coaxial HPGe detector. The segmentation scheme is indicated on the left, the detector housing and the preamplifier arrangement can be seen on the right. 

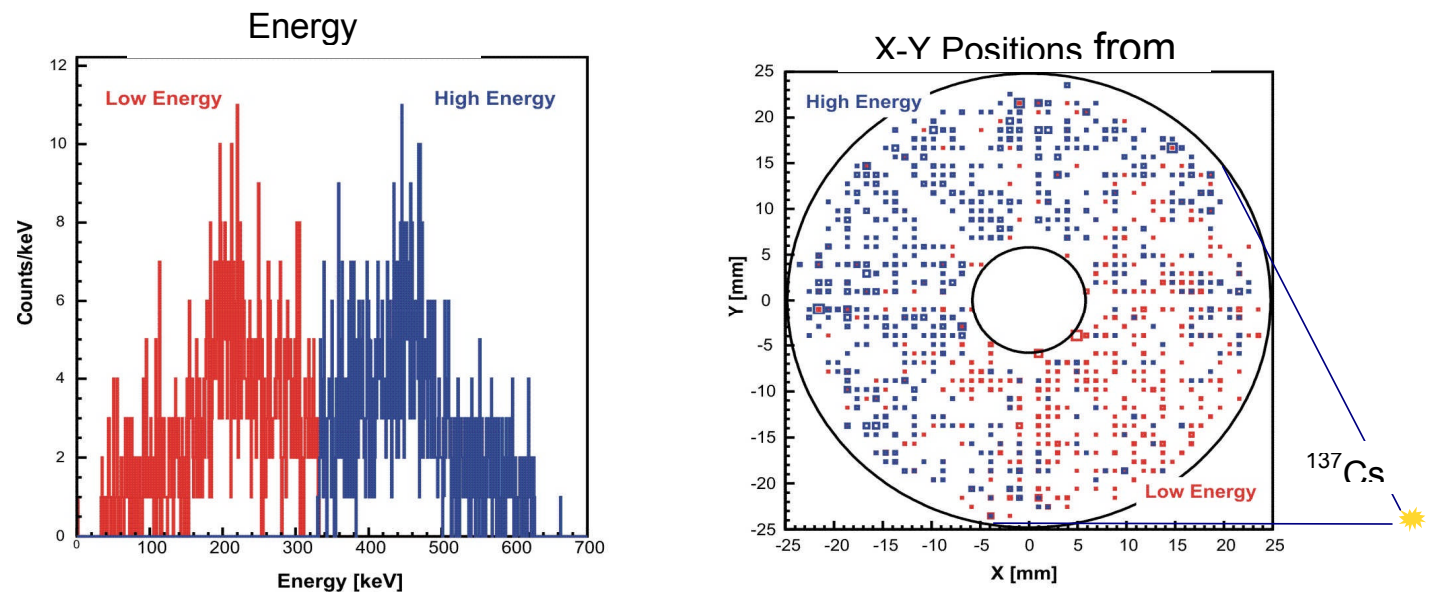

Figure 37: Energies (left) and positions (right) identified and determined by signal decomposition calculations after illuminating the detector with a ${ }^{137} \mathrm{Cs}$ source as indicated on the right. The red boxes indicate the low energies and therefore the first of the two interactions.

To extract the number of interactions, their energies, and their three-dimensional positions the measured pulse-shapes of each segment is decomposed into sets of calculated signals. Analyzing the signal shapes not only from the charge-collecting contact but also from all adjacent segments it is possible to determine the threedimensional position of the interaction with much more accuracy than the size of the segment itself. For example, it was shown that a position resolution of about $0.5 \mathrm{~mm}$ is achievable at $374 \mathrm{keV}$ [87]. To provide the signal shapes for this analysis, each preamplifier output is directly fed into a fast and high-resolution waveform digitizer and all further processing and feature extraction is done on a computer. This includes the determination of the energies, event selection, and ultimately the signal decomposition minimization procedure. To demonstrate Compton imaging with this approach a ${ }^{137} \mathrm{Cs}$ source was placed $2 \mathrm{~m}$ away from the crystal as shown in Figure 38 preliminary event selection was performed and only events with two segment hit were processed assuming that only one interaction took place in one segment.

Positions and energies of the to identified interactions during this experiment are shown in Figure 38. On the left, the energies of the first and second interactions are shown. The sequence was determined by taking the smaller of the two energies as the first interaction which is much more likely than the opposite sequence given the Klein-Nishina cross section formula and the given geometry. As it can be seen from the associated position shown on the right, most of the first interaction are closer to the source as expected. 

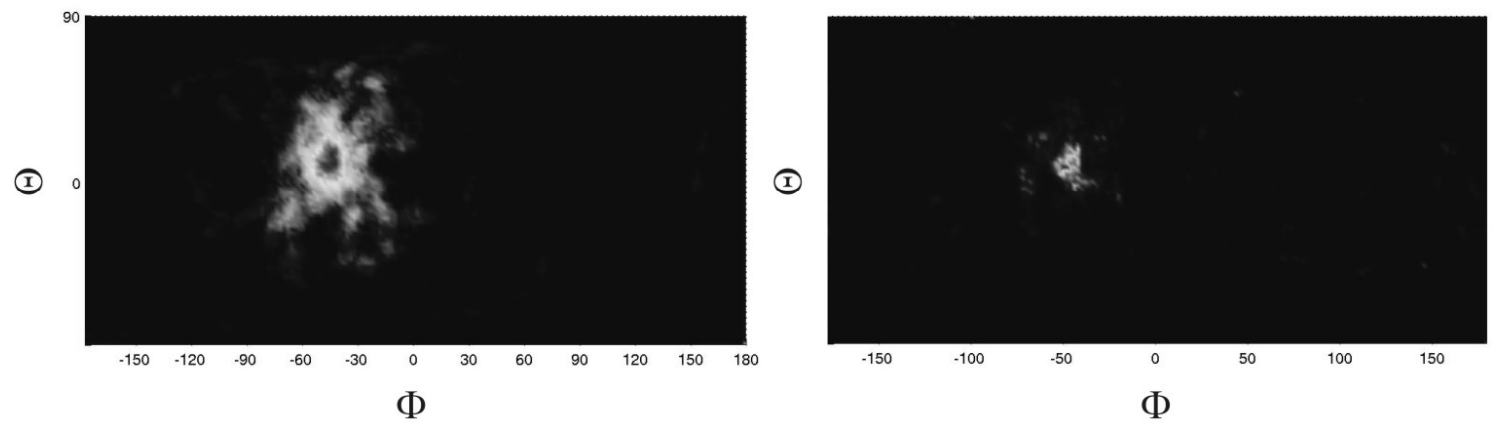

Figure 38: Images deduced by the measured energies and positions of two interactions in the detector. The left image was determined with simple cone back projection, the image on the right was obtained with an iterative list-mode maximum-likelihood method.

Using the energy and position information, Compton images were produced as shown in Figure 39. On the left, a simple backprojection is shown, on the right the same data were processed by a list-mode maximum likelihood procedure by taking the system responses such as a position resolution of $3 \mathrm{~mm}$ (FWHM) into account. An angular resolution of 5 degrees was demonstrated at an energy of $662 \mathrm{keV}$. The relative efficiency was determined to be $0.3 \%$. This efficiency expresses the fraction of gamma rays, which can be imaged out of the total gamma ray flux, which is emitted into the solid angle of the detector. It was also shown that with more advanced signal decomposition and event selection schemes which have been demonstrated for other applications [87] it should be possible to increase the efficiency to about $5 \%$ to achieve 5 degrees angular resolution at an energy of $662 \mathrm{keV}$.

The advantage of such a system is the high overall efficiency and excellent energy resolution which characterizes coaxial HPGe detectors and the potentially good Compton imaging performance with only about 40 channels. Two-dimensionally segmented coaxial HPGe detectors have been built to diameters of $7 \mathrm{~cm}$ and lengths of $14 \mathrm{~cm}$ resulting in very high efficiency [88]. The maximum dimensions which can be achieved in fabricating this type of detectors is ultimately limited by the magnitude and the difference of the impurity concentration in the grown crystal. Considering the recent increase in the demand due to the ability to mechanically cooled HPGe detectors, it appears possible to build a mechanically cooled, coaxial HPGe Compton imager. In addition to the good angular resolution and imaging efficiency, the large FOV is noteworthy for this particular implementation. Due to the coaxial shape, almost all directions in $4 \pi$ are equally represented and all high-sensitivity Compton imaging in $4 \pi$. 


\section{The LLNL single DSSD HPGe Compton imager}

An alternative configuration of positionsensitive HPGe detectors is a planar geometry with orthogonal strips on each side, the so-called double-sided strip detector (DSSD) configuration. On the figure to the right, a DSSD HPGe detector is shown as developed and built at LNNL. The detector system consists of a cryostat with the DSSD detectors, readout components, feedthroughs, and preamplifiers. This DSSD HPGe detector has an active area of about $50 \mathrm{~cm}^{2}$, which is divided into 38 strips with a pitch width of $2 \mathrm{~mm}$ and a gap of $0.5 \mathrm{~mm}$ in between the strips. The thickness is $11 \mathrm{~mm}$. These detectors can be built to about $20 \mathrm{~mm}$ limited by the impurity concentration, which defines the depletion and therefore the operational voltage. The operational

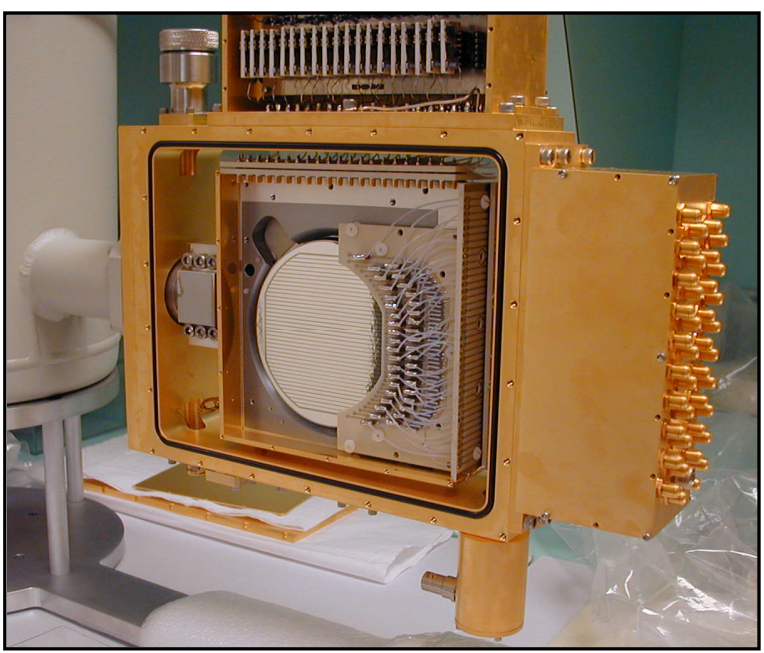

Figure 39: A DSSD HPGe detector in a cryostat including readout components and preamplifiers. voltage can not be increased indefinitely since potentially the leakage current degrades the noise performance and therefore the energy resolution to unacceptable levels. The maximum diameter is limited due the maximum wafer diameter, which is currently 10 $\mathrm{cm}$. Currently, a guard ring of about $5 \mathrm{~mm}$ is needed at the outside edge to separate the detector surface effects from the bulk properties.

The DSSD configuration is preferred over for example a pixellated configuration due to the number of channels needed for two-dimensional segmentation. For example, as shown in the figure above 76 segments are needed in DSSD configuration while $38 \times 38=1444$ channels would have been required when used in pixellated configuration with the same pitch size of $2 \mathrm{~mm}$. As DSSD system all the channels can be readout and processed individually with high-resolution components such as the FET. In the pixilated configuration, custom made ASICs need to be developed which are much more complex, particularly if high resolution is required.

As with the coaxial HPGe configurations, the two-dimensional segmentation along with pulse-shape processing provides not only the third position dimensions (the depth in DSSD geometry) but can be used to improve the lateral position resolution as well. This is illustrated in Figure 41. The time difference of opposing signals is used to derive the depth of the interaction, the induce signal on adjacent strips provide additional information on the position perpendicular to the depth. In this way, a position resolution of better than $0.5 \mathrm{~mm}$ was achieved at a gamma-ray energy of $122 \mathrm{keV}$ [86]. 

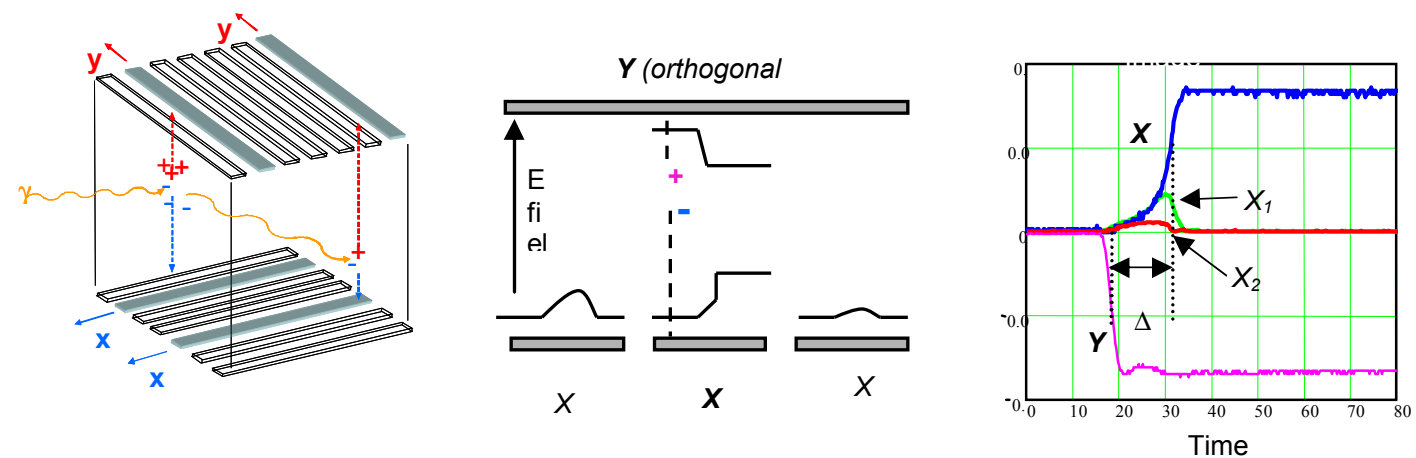

Figure 40: Illustration of the generation and shapes of pulses in a DSSD system.

One of the available DSSD HPGe systems were used to demonstrate Compton imaging down to $344 \mathrm{keV}$ with about $7 \mathrm{deg}$. angular resolution. The resolution improves for higher gamma-ray energies. Energy resolution of $1.1 \mathrm{keV}$ at $60 \mathrm{keV}$ and $1.6 \mathrm{keV}$ at 662 $\mathrm{keV}$ has been achieved for individual strips by digitally processing the signal shapes.

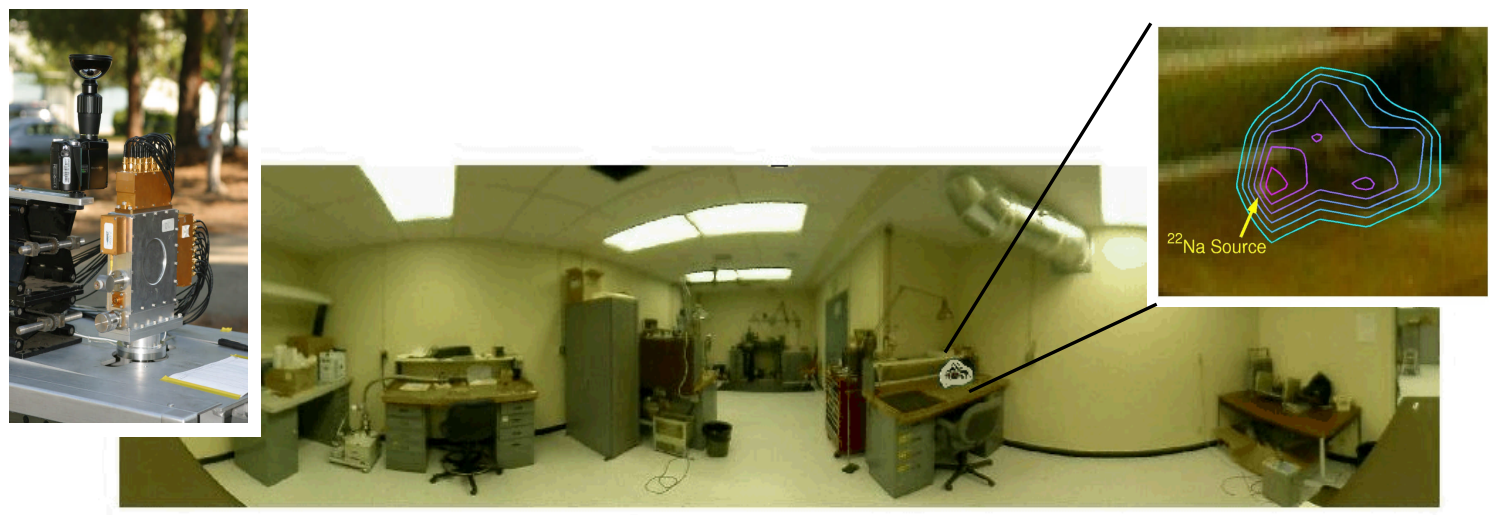

Figure 41: Panoramic photograph overlaid with a $4 \pi$ gamma-ray image. On the left, the photocamera with its fish lens and the DSSD HPGe detector is shown. On the right, the close up view of the gamma-ray image with the source location in a source container is shown. The source was correctly identified as ${ }^{22} \mathrm{Na}$.

As with the coaxial HPGe detector, it is possible to provide high-sensitivity gamma-ray imaging in $4 \pi$ with a DSSD HPGe detector. Figure 42 illustrates the ability to overlay a panoramic visual image with a $4 \pi$ gamma-ray image. In this way, sources can be detected, localized through Compton imaging, and identified through the high-resolution spectroscopy possible with Ge detectors.

This feature could be very important in the monitoring of nuclear facilities to prevent clandestine removal of nuclear materials in a way which is not possible with visual imaging alone. 


\section{Next Compton Telescope}

The Next Compton Telescope (NCT) is again an Compton imaging system designed for balloon-borne astrophysical measurements [84]. In addition, it serves as a precursor of the next large-scale gamma-ray telescope by NASA, the Advanced Compton Telescope (ACT). NCT is illustrated in Figure 43. It is designed to contain 12 DSSD HPGe detectors, each with about $50 \mathrm{~cm}^{2}$ active area, which is divided by 37 strips of $2 \mathrm{~mm}$ pitch size and a thickness of $15 \mathrm{~mm}$. As in the system mentioned before, the goal is to achieve an energy resolution of about $2 \mathrm{keV}$ at $60 \mathrm{keV}$ and an angular resolution of a few degrees at $1000 \mathrm{keV}$ with a large FOV. A two-detector version of NCT was successfully launched last summer and the data analysis is underway.
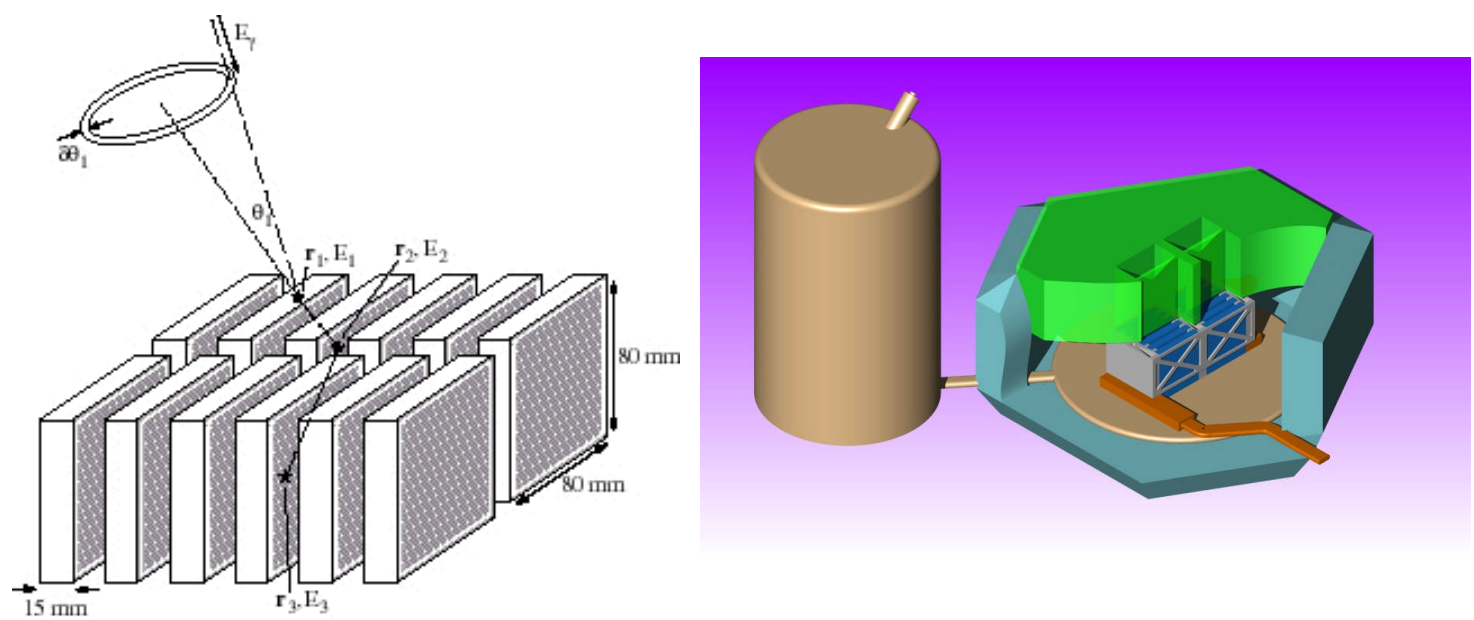

Figure 42: In the full implementation, NCT will consist of 12 DSSD HPGe detectors arranged as shown on the left. The whole instrument as it will be mounted on the gondola of the balloon is shown on the right.

\subsubsection{The Si+Ge Compton imager}

The Ge-only Compton imaging systems discussed above are very powerful instruments due to their efficiency, energy resolution, compactness, and angular resolution, particularly above $500 \mathrm{keV}$. However, to achieve improved Compton imaging sensitivity in terms of resolution and efficiency below $500 \mathrm{keV}$, a lower- $Z$ material is required to induce Compton imaging to lower energies, to separate the first two interactions and to reduce the degradation due to the intrinsic momentum of the Compton electron (Compton profile).

Monte-Carlo simulations have shown that the best currently possible configuration to for Compton imaging for energies ranging from about $150 \mathrm{keV}$ to about $3000 \mathrm{keV}$ is a hybrid system built of two different, position-sensitive, and high resolution detectors of low- $Z$ and higher- $Z$ material. The lower $Z$ component induces the Compton scattering particularly for low energies, while the higher $Z$ component stops the gamma ray or induces Compton scattering and stops the gamma ray of higher energy. 


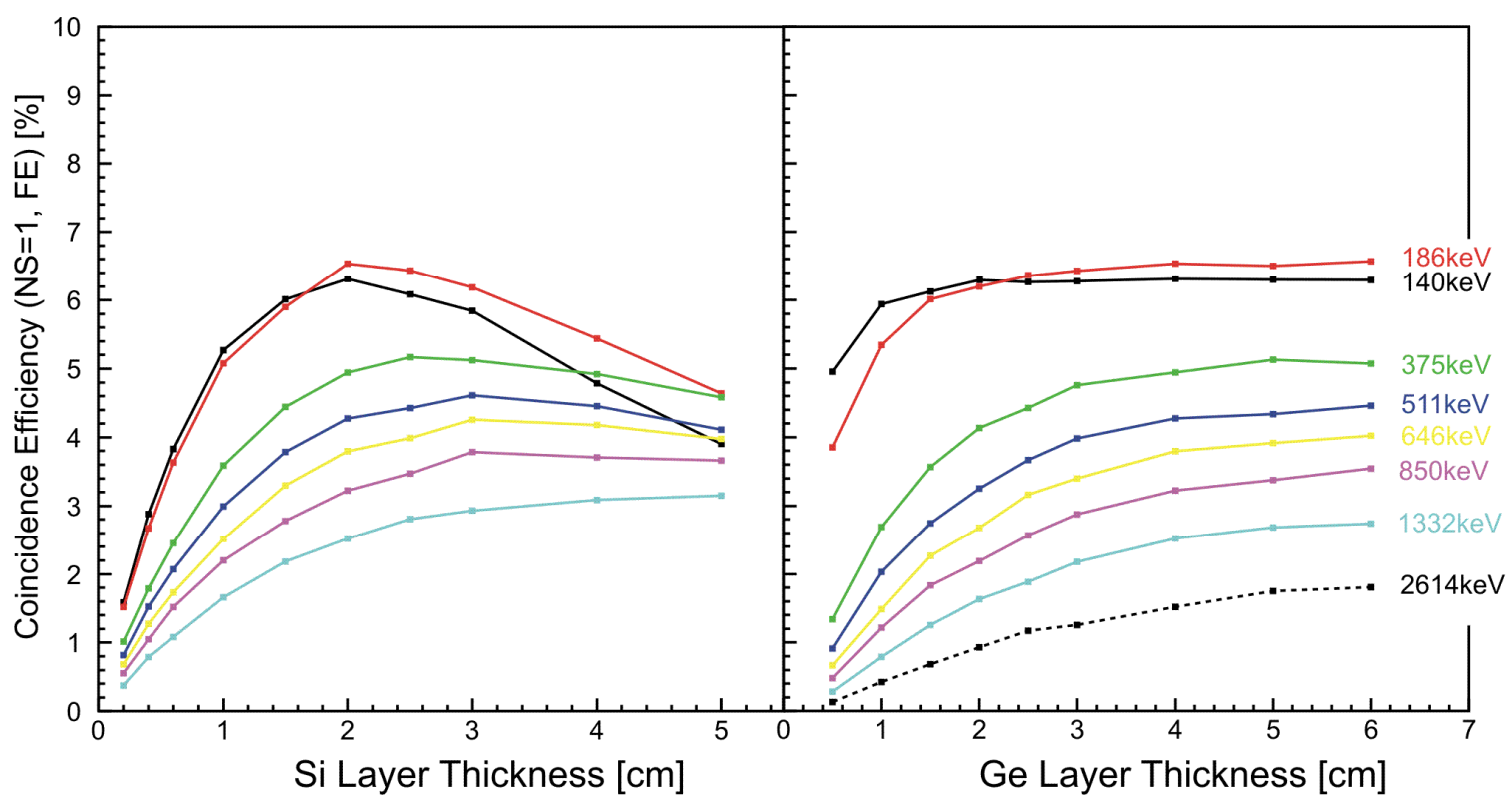

Figure 43: Calculated efficiencies for a hybrid imager as shown in Figure 28 as a function of detector thicknesses. On the left, the thickness of the Si layer was varied, on the right, the thickness of the Ge layer.

Figure 44 shows calculated efficiencies for a hybrid imager as shown in Figure 29 as a function of detector thicknesses. Plotted is the efficiency for both detectors to be hit, the full energy was deposited, and the requirement that only one and the first interaction took place in the Si layer. The left hand figure shows efficiencies as a function of the Si thickness for a range of gamma-ray energies. The thickness of the Ge layer was assumed to be $4 \mathrm{~cm}$. The right hand figure shows efficiencies as a function of the Ge layer thickness assuming a $2 \mathrm{~cm}$ thick Si layer in front of it. In both figures, the distance between the $\mathrm{Si}$ and the Ge layer was assumed to be $3 \mathrm{~cm}$ for the calculations.

With regard to the Si thickness it can be seen that below $500 \mathrm{keV}$ it is not possible to gain significantly in efficiency for Si thicknesses larger than $20 \mathrm{~mm}$. For higher energies we could gain slightly more by increasing the thickness beyond $20 \mathrm{~mm}$. However, the gain e.g. $20 \%$ at $1.33 \mathrm{MeV}$ when increasing the thickness from $20 \mathrm{~mm}$ to $50 \mathrm{~mm}$ - is only marginal considering the additional complexity required to build an instrument with 2.5 times more Si thickness. More importantly, for gamma-ray energies above $500 \mathrm{keV}$ the Ge detectors by themselves are becoming efficient Compton imaging instruments even without requiring the coincidence and the first interaction in the Si detectors. The Si detectors are primarily used to enable efficient Compton imaging below about $500 \mathrm{keV}$. Increasing the thickness beyond $20 \mathrm{~mm}$ would actually reduce the efficiency more energies below $350 \mathrm{keV} .20 \mathrm{~mm}$ Si can be achieved by two $10 \mathrm{~mm}$ thick Si(Li) detectors. $10 \mathrm{~mm}$ is currently the maximum thickness which can be achieved for $\mathrm{Si}(\mathrm{Li})$ detectors. With regard to the Ge detector thickness, the gain in efficiency beyond $30-40 \mathrm{~mm}$ thickness is not significant anymore and does not justify to increase the complexity and operational constraints by adding an additional layer. 30-40 $\mathrm{mm}$ can be achieved with two 15-20 mm thick Ge detectors. 
It is interesting to note that efficiencies of a few percent can be achieved with such a setup for energies ranging from $186 \mathrm{keV}$ to $2614 \mathrm{keV}$ and that the efficiency does not strongly depend on the energy, in contrast to collimator-based systems. For larger-area systems, which are built of $\mathrm{Si}+\mathrm{Ge}$ detector modules, the relative efficiency can be increased to $10-20 \%$ by adding gamma ray interaction from different modules.

Figure 45 shows the first generation $\mathrm{Si}+\mathrm{Ge}$ Compton imaging system mounted on a cart to provide the ability to move the system. The instrument consists of one $64 \times 64 \times 10$ $\mathrm{mm}^{3} \mathrm{Si}(\mathrm{Li})$ detector in DSSD configuration with 32 strips on each side with a pitch size of $2 \mathrm{~mm}$ and a DSSD HPGe detectors as shown in Figure 40. Each detector is mounted in its own cryostat. The detector surfaces are $6 \mathrm{~cm}$ apart. All 144 channels are directly fed into a waveform digitizer system, which digitizes the signal shape at $100 \mathrm{MHz}$ with 14 bit resolution. Energy resolution values of

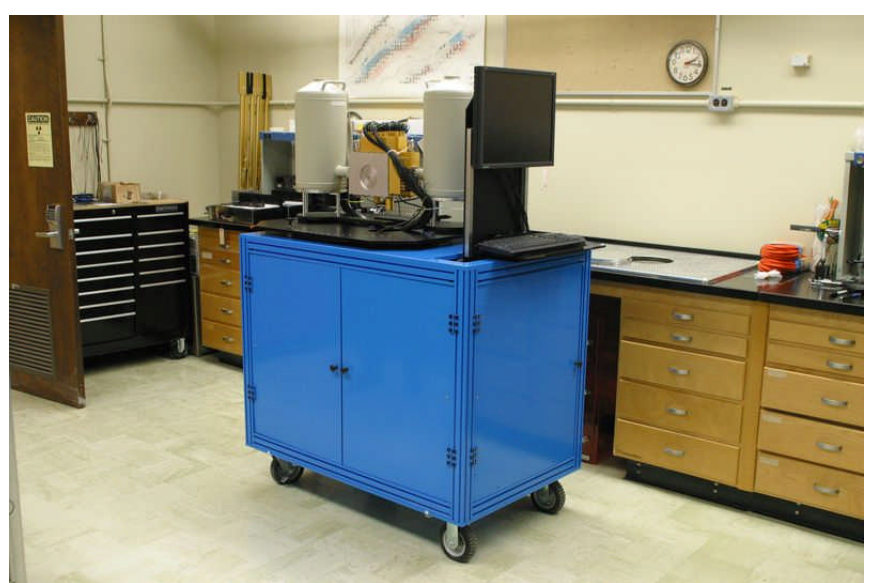

Figure 44: The first generation $\mathrm{Si}+\mathrm{Ge}$ Compton imaging system (CCI-1), which mounted on a cart. about 1.8 (1.4) keV and $2.2(2.0) \mathrm{keV}$ has been achieved per strip at $60 \mathrm{keV}$ and $662 \mathrm{keV}$, respectively for the $\mathrm{Si}(\mathrm{Li})$ (HPGe) detector in this setup. Figure 46 shows positions and a corresponding image, which were measured at $779 \mathrm{keV}$.

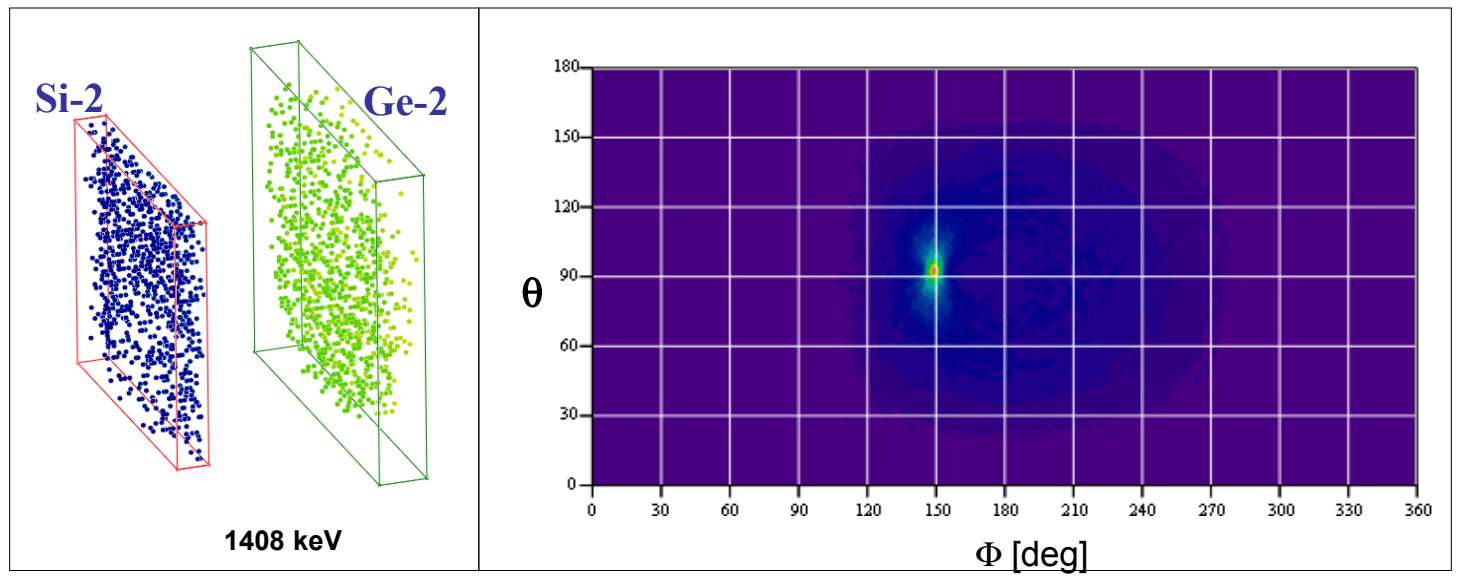

Figure 45: Illustration of gamma-ray interactions in CCI-1 (left) and the resulting backprojected image. Shown are experimental data obtained with a ${ }^{152} \mathrm{Eu}$ source located at 30 deg. and an energy of $779 \mathrm{keV}$. 


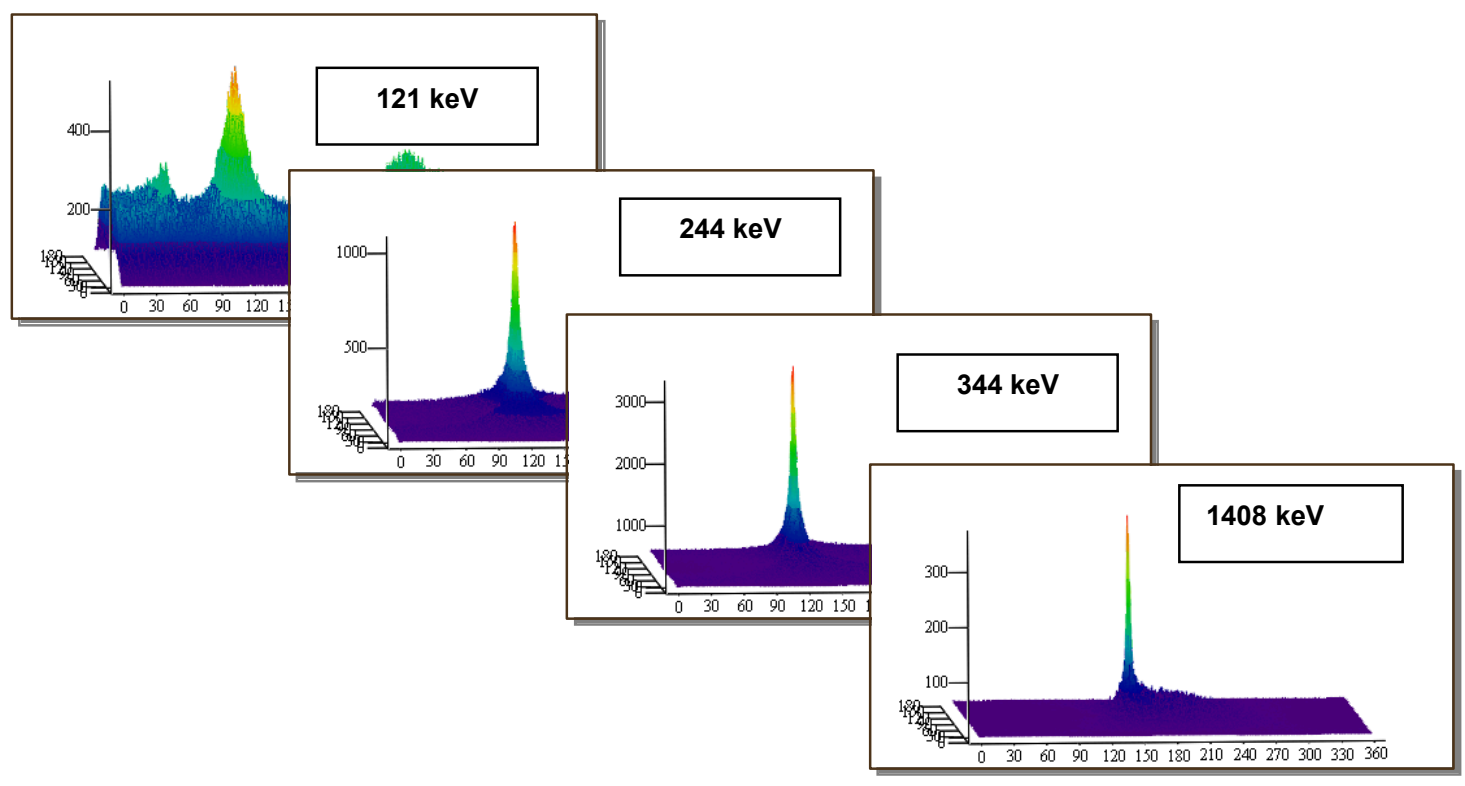

Figure 46: Simple back-projected gamma-ray images obtained with CCI-1 for a range of gamma-ray energies from a ${ }^{152} \mathrm{Eu}$ source.

Figure 47 shows simple back projected images measured with CCI-1 for a energies ranging from $121 \mathrm{keV}$ to $1408 \mathrm{keV}$ to demonstrate the Compton imaging capability. Figure 48 shows measure Angular Resolution Measure (ARM) values. It also shows theoretical values and the contribution by the Doppler broadening or Compton profile, energy resolution and position resolution. The overall discrepancy of about $0.5 \mathrm{deg}$ between the measured and the calculated ARM values are likely due to a misalignment of the relative detector positions in the order of $0.5 \mathrm{~mm}$.

Currently, a second generation of this type of hybrid Compton imagers with two large DSSD Si(Li) detectors with an overall thickness of $20 \mathrm{~mm}$ and two large DSSD HPGe detectors with an overall thickness of $30 \mathrm{~mm}$ is being built. The efficiency should be comparable to the values shown in Figure 44. It should be able to acquire data with up to $10 \mathrm{kHz}$ event rates which will enable the demonstration of Compton imaging and this approach for many potential applications, even in complex radiation fields. 

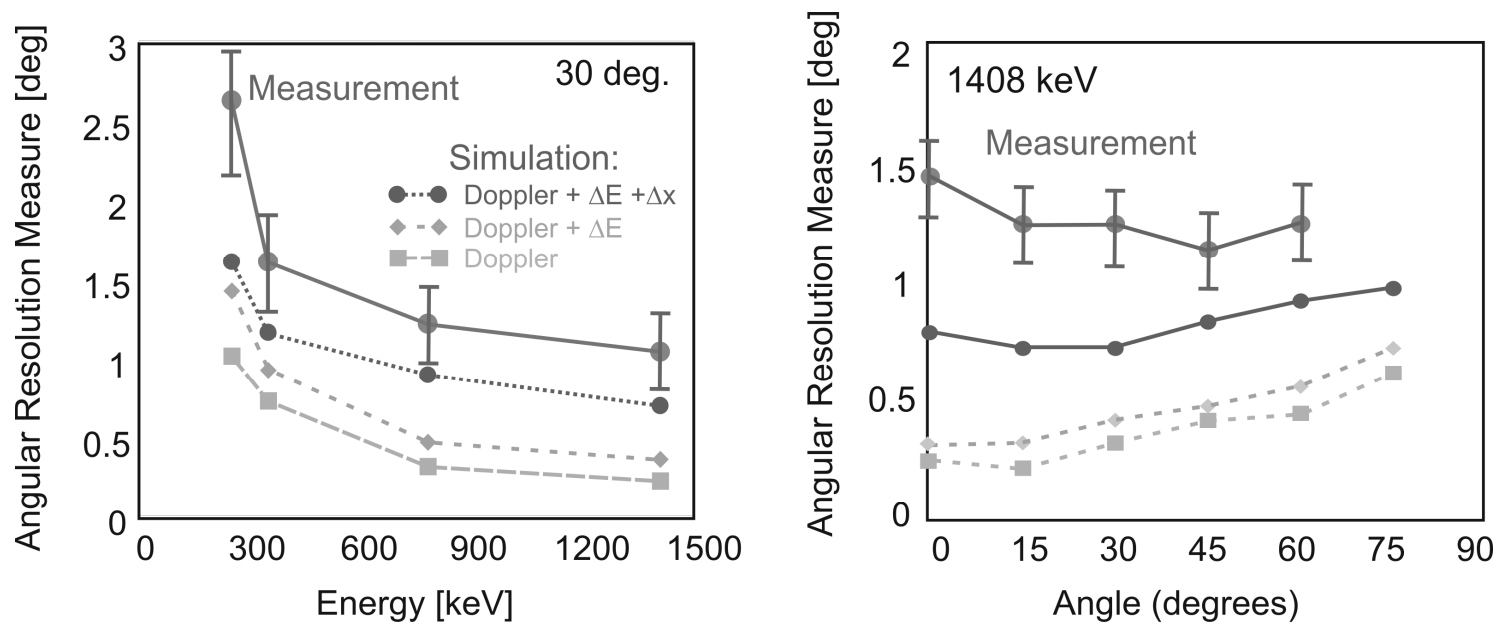

Figure 478: Measured ARM values for different energies (left) and source locations (right) in comparison with calculations.

\subsubsection{Concluding remarks}

The concept of Compton imaging is about 40 years old, however, only recent advances in detector technology, electronics, and computing allow us to realize the full potential of Compton imaging. That is reflected in the increasing umber of efforts to develop Compton imaging systems for a variety of applications ranging from astrophysics to biomedical imaging and homeland security.

On one hand, large systems are being pursued, e.g. for the Advanced Compton Telescope (ACT) for astrophysics applications, on the other hand, pocket-sized system are being developed for homeland security applications. Currently, the most promising technologies are based on three-dimensionally position-sensitive semi-conductors either built of cryogenically cooled $\mathrm{Si}$ and Ge detectors or room-temperature operational detectors built of Si or CZT. This is due to the fact that currently the highest sensitivity can be achieved in these systems by combining high efficiency with high energy and three-dimensional position resolution. Including a new generation of three-dimensional position sensitive solid-state scintillator detectors at least as secondary capture detector can be envisioned, however, current scintillators degrade the overall achievable sensitivity significantly. Gas-based system e.g. built of Ar or Xe and operated as Time Projection Chamber are very appealing since in principle provide the track of Compton electrons to measure the direction of the incident gamma ray directly and not only the cone of possible directions. However, the trade-off here is the ability to observe a sufficiently long electron track which requires low density (e.g. pressure of about $1 \mathrm{~atm}$ ) and the ability to actually induce interactions and to detect the gamma ray at all which requires high density (e.g. pressures of up to $50 \mathrm{~atm}$ which still is associated with small densities $\rho<1 \mathrm{~g} / \mathrm{cm}^{3}$ ). Compton imaging systems based on liquid scinitllators and also operated as TPC are appealing due to its potentially scalability. However, the complexity 
in operating a large volume liquid systems remains a significant challenge and systems with high position and energy resolution remain to be developed and demonstrated.

Another approach can be envisioned by using small scintillating fibers. This approach is appealing since such a system can potentially be built to large dimensions and due the low density and low $\mathrm{Z}$ materials involved could enable electron tracking. The limiting factors here however, are the finite light production per energy loss and the efficient and fast light readout.

Finally, we want to point out that a hybrid system built of a Compton imager and a coded aperture can be envisioned, too. A relatively thin $(\sim 1 \mathrm{~cm})$ coded aperture can be mounted in front of a Ge or CZT based Compton imager. In this way, the coded aperture provides the imaging capability for energies below about $1 \mathrm{MeV}$ (and as low as about $20 \mathrm{keV}$ ) and the Compton imager for energies above $1 \mathrm{MeV}$. The coded aperture will reduce the fieldof-view and will increase the overall size and weight, however, allows to image gammarays below $100 \mathrm{keV}$ which will be difficult to achieve with Compton imaging systems alone.

In table 3 we summarize some of the performance and engineering characteristics of the Compton imaging systems which have been discussed in the previous sections.

\begin{tabular}{|l|l|l|l|l|l|l|}
\hline System & FOV & $\begin{array}{l}\Delta \mathrm{E} \\
(662 \mathrm{keV})\end{array}$ & $\begin{array}{l}\Delta \theta \\
{[\mathrm{deg}]}\end{array}$ & $\begin{array}{l}\text { Weight } \\
\text { ratio }\end{array}$ & $\begin{array}{l}\text { E-range } \\
{[\mathrm{keV}]}\end{array}$ & Portability \\
\hline $\begin{array}{l}\text { Nal } \\
\text { Comptel }\end{array}$ & $2 \pi$ & $10 \%$ & $<10$ & & $>500$ & - \\
\hline $\begin{array}{l}\text { Si-Nal } \\
\text { Csprint }\end{array}$ & $2 \pi$ & $8 \%$ & $<10$ & & $>100$ & \\
\hline $\begin{array}{l}\text { LXe } \\
\text { LXeGrit }\end{array}$ & $2 \pi$ & $3 \%$ & $<10$ & $>50 \%$ & $>500$ & \\
\hline $\begin{array}{l}\text { Si-CsI } \\
\text { MEGA/TIGRE }\end{array}$ & $2 \pi$ & $6 \%$ & $<10$ & & $>100$ & \\
\hline Ar-CsI & $2 \pi$ & $8 \%$ & $<15$ & & $>100$ & \\
\hline $\begin{array}{l}\text { Si-CdTe } \\
\text { NEXT }\end{array}$ & $2 \pi$ & $<2 \%$ & $<5$ & & $>100$ & \\
\hline $\begin{array}{l}\text { Si-Ge } \\
\text { LLNL }\end{array}$ & $2 \pi$ & $<0.5 \%$ & $<2$ & & $>100$ & \\
\hline Si-only & $4 \pi$ & $<2 \%$ & $<10$ & $>50 \%$ & $>100$ & \\
\hline $\begin{array}{l}\text { CZT-only } \\
\text { Polaris }\end{array}$ & $4 \pi$ & $<2 \%$ & 15 & $>70 \%$ & $>500$ & \\
\hline Ge-only & $4 \pi$ & $<0.5 \%$ & $<5$ & $>50 \%$ & $>300$ & \\
\hline & & & & & & \\
\hline
\end{tabular}

Table 3: Characteristics of Compton imaging system types.

\footnotetext{
${ }^{6}$ Comptel was the first Compton imaging system that was deployed.

${ }^{7}$ Ge-only Compton imagers have been demonstrated in two different implementations: planar DSSD and two-dimensionally segmented, coaxial Ge detectors. Planar DSSD system have been implemented by NRL, LLNL, SSL, and at the Univ. Tokyo. The coaxial imager was demonstrated by LLNL.
} 


\section{BIBLIOGRAPHY}

[1] H.O. Anger, Rev. Sci. Instr. 29 (1958) 27

[2] J.A. Sorenson and M. Phelps, Physics in Nuclear Medicine (second ed.), W. B.

Saunders Company, Philadelphia, PA (1987).

[3] K. P. Ziock et al., IEEE Trans. Nucl. Sci., $51-5$ (2004) 2238,

K.P. Ziock et al., IEEE NSS Conference Record, 2004, Pages: 322- 326

[4] Pohl

[5] K.P. Ziock et al., Nucl. Instr. Meth. Phys. Res. A, Vol. 505 (2003) 420

[6] N.H. Clinthorne, et al., IEEE Nucl. Sci. Symp. Conf. Rec. 2 (1997) 788

[7] J.W. LeBlanc et al., IEEE Trans. Nucl. Sci. 45,3 (1998) 943

[8] A.O. Hero, J.A. Fessler, and M. Usmann, IEEE Trans. Nucl. Sci. 44,8 (1996) 2026

[9] D.L. Windt et al., Appl. Opt. 42 (2003), p. 2415

[10] M.J. Pivovaroff et al., Journal of Nucl Med. 43 (2002) 231

[11] Heft

[12] V. Schoenfelder, et al., IEEE Trans. on Nuclear Science, NS-31, 766-770, 1984

[13] V. Schoenfelder et al., Astrophys. J. Suppl. 86, pp.657-692, 1993.

[14] R. C. Rohe and J.D. Valentine, IEEE Trans. Nucl. Sci., Vol.43, no.6,p.3256-3263 1996

[15] Y.F. Du, Z. He, G.F. Knoll, D.K. Wehe, W. Li, Nucl. Instr. Meth. Phys. Res. A, Vol. 457, pp. 203-211, 2001

[16] R.A. Kroeger, W.N. Johnson, J.D. Kurfess, B.F. Phlips, E.A. Wulf, IEEE Trans. Nuc. Sci., 49,pp. 1887-1892, 2002

[17] Boggs SE, Coburn W, Smith DM, et al., NEW ASTRONOMY REVIEWS 48 (14):pp. 251-255, 2004

[18] J. Bernabeu et al., Nucl. Instr. Meth. Phys. Res. A, 527, pp. 58-61, 2004

[19] A. Studen, D. Burdette, E. Chesi, V. Cindro, N. H. Clinthorne, W. Dulinski, J.

Fuster, L. Han, H. Kagan, C. Lacasta et al., NIM-A,Volume 531, Issues 1-2, 21

September 2004, Pages 258-264

[20] ] A. Takada, K. Hattori, H. Kubo, K. Miuchi, T. Nagayoshi, H. Nishimura, Y.

Okada, R. Orito, H. Sekiya, A. Tada and T. Tanimori, NIM-A, Volume 546, Issues 1-2, 1 July 2005, Pages 258-262

[21] T. Niedermayr et al., Nucl. Instr. Meth. 553-3 (2005) 501

[22] K. Vetter, M. Burks, L. Mihailescu, Nucl. Inst. Meth. A 525 (2004) 322

[23] R.W. Todd, J.M. Nightingale and D.B. Everett,Nature, 251, p.132-134, 1974

[24] V. Schoenfelder, A. Hirner and K. Schneider, Nucl. Instr. and Meth. in Phys. Res., 107 , p. $385-394,1973$

[25] http://gsc.nrcan.gc.ca/gamma/hist_e.php

[26] ARAM

[27] Pinhole w/ $1 \mathrm{~mm}$ resolution

[28] D.P. McElroy et al., IEEE Trans. Nucl. Sci. 49 (2002) 794

[29] H. Dicke, Astrophys. J. 153, L101 (1968).

[30] J. G. Ables, Proc. Astron. Soc. Aust. 4, 172 (1968). 
[31] H. H. Barrett and F. A. Norrigan, Appl. Opt. 12, 2686 (1973).

[32] C. M. Brown, Ph.D. thesis, "Multiplex Imaging and Random Arrays," U. Chicago (1972).

[33] R. L. Blake, A. J. Burek, E. E. Fenimore, and R. Puetter, Rev. Sci. Instrum. 45, 513

(1973). (From "Coded aperture imaging with uniformly redundant arrays" E. E. Fenimore and T. M. Cannon)

[34] GRIS/ Ziock

[35] W. R. Cook, M. Finger, T. A. Prince, and E. C. Stone, IEEE Trans. Nucl. Sci., vol.

31, pp. 771-775, Feb. 1984

[36] Hamamatsu Photonics K.K., Toyooka-Village, Iwata-gun, Shizuoka-ken 438-0193, Japan

[37] RadCam by Radiation Monitoring Devices, Watertown, MA

[38] Rexon Components, Inc., Cleveland, $\mathrm{OH}$

[39] L. Nakae and K. P. Ziock, SPIE Conf. 3768: Hard X-Ray, Gamma-Ray, and Neutron

Detector Physics, R. B. James and R. C. Schirato, Eds. Bellingham, U.K.: SPIE, 1999, pp. 173-182

[40] K.P. Ziock et al., Nucl. Instr. Meth. Phys. Res. A, Vol. 505 (2003) 420

[41] K. P. Ziock, L. Madison, B. R. McGinnis, R. R. Royce, and A. Brown, in Proc. 36th

Annu. INMM Meet., Palm Desert, CA, July 1995

[42] LAI /Ziock

[43] G. Vedrenne et al., Astronomy \&Astrophysics 411, L63-L70 (2003)

[44] Naya, J., Jean, P., Buckolt, J., et al., NIM A, 368, 832

[45] Jean, P., Vedrenne, G., Roques, J. P., et al. 2003, A\&A, 411, L107

[46] Gehrels, N. 1985, NIM A, 239, 324

[47] Gehrels, N. 1992, NIM A, 313, 513

[48] Sanchez, F., Chato, R., \& Gasent, J. L. 2000, SPI Mask STM/QM Transparency Test Report, IN-SP-UV-RPT-049

[49] E. Fenimore and T. Cannon, Appl. Opt., vol. 20, no. 10, pp. 1858-1864, 1981

[50] R. Accorsi, F. Gasparini, and R. C. Lanza, IEEE Trans. Nucl. Sci., vol. 48, no. 6, pp. 2411-2417, Dec. 2001

[51] Mertz, Proc. Symp. On Modern Optics, Brooklyn, March 1967 (1968)

[52] H. W. Schnopper, R. I. Thompson, S. Watt, Space Sci. Rev. 8 (1968) 534-542 [53]

[53] Cruise \& Wilmore 1975

[54] Staubert 1983

[55] Ubertini 1985

[56] Nishimura 1978

[57] T. A. Prince, G. J. Hurford, H. S. Hudson, C. J. Cranell, Solar Physics vol. 118 (1988) pp. 269-290

[58] G. J. Hurford, E. J. Schmahl, R. A. Schwartz, A. J. Conway, M. J. Aschwanden, A. Csillaghy et al., Solar Physics vol. 210 (2002) pp. 61-86

[59] D. M. Smith, G. J. Hurford, S. E. Boggs, New Astronomy (preprint). 26 Sept. (2003)

[60] J. R. Kilner, G. H. Nakano, SPIE Proc. vol. 1159 (1989) p. 27

[61] L. E. Orwig et al., Max 1991 Workshop 2: Developments in Observations and Theory for Solar Cycle vol. 22 (1989) p. 143

[62] R. Lieu, K. J. Beurle, A. R. Engel, J. J. Quenby, J. Phys. E: Sci. Instrum. vol. 17 (1984) pp. 577-580 
[63] D.L. Gunter, 2005, LLNL report, UCRL

[64] A. Zoglauer et al., Proc. SPIE 4851 (2003)

[65] R. Ribberfors, Phys. Rev. B 12, p.2067, 1975

[66] Biggs, L.B. Mendelsohn, J.B. Mann, Atom. Data Nucl. Data Tables 16, 1975 [67]

[68] D. Brusa et al., Nucl. Instr. and Meth. 379 (1996), p. 167

[69] Aprile E, Curioni A, Giboni KL, et al., NEW ASTRONOMY REVIEWS 48 (1-4), pp. 257-262, 2004

[70] A. Takada, K. Hattori, H. Kubo, K. Miuchi, T. Nagayoshi, H. Nishimura, Y. Okada, R. Orito, H. Sekiya, A. Tada and T. Tanimori, NIM-A, Volume 546, Issues 1-2, 1 July 2005, Pages 258-262

[71] E. Aprile, M. Suzuki, IEEE Trans. Nucl. Sci. 36 (1989) 311

[72] T.J. O’Neilly et al., IEEE Trans. Nucl. Sci. 50 (2003) 251

[73] G. Kanbach et al., Proc. SPIE 4851 (2003) 1209

[74] R. Orito et al., Nucl. Instr. Meth. Phys. Res. A 525 (2004) 107

[75] T, Mitani et al., IEEE Trans. Nucl. Sci. 51 (2004) 2432

[76] 3 Compton NRL

[77] A. Morselli et al., Nucl. Instr. Meth. Phys. Res. A 530 (2004) 158

[78] C.E. Lehner et al., IEEE Trans. Nucl. Sci. 51 (2004) 1618

[79] P. Luke, IEEE Trans. Nucl. Sci. 42 (1995) 207

[80] Z. He et al., Nucl. Instr. Meth. Phys. Res. A 422 (1999) 173

[81] G.J. Royle et al., Nucl. Instr. Meth. Phys. Res. A 348 (1994) 623

[82] Japan CI/ Ge

[83] E.A. Wulf et al., IEEE Trans. Nucl. Sci. 50 (2003) 1183

[84] S.E. Boggs et al., Proc. SPIE 4851 (2002) 1221

[85] T. Niedermayr, et al., Nucl. Instr. Meth. A 553 (2005) 501

[86] K. Vetter, M. Burks, L. Mihailescu, Nucl. Inst. Meth. A 525 (2004) 322

[87] K. Vetter, et al., Nucl. Instr. Meth. A452, 223 (2000)

[88] J. Gerl, priv. comm. 